\title{
Echocardiography : modern evaluation of cardiac structure and function
}

Citation for published version (APA):

Kaufmann, B. A. (2016). Echocardiography : modern evaluation of cardiac structure and function.

[Doctoral Thesis, Maastricht University]. Maastricht University. https://doi.org/10.26481/dis.20160311bk

Document status and date:

Published: 01/01/2016

DOI:

10.26481/dis.20160311bk

Document Version:

Publisher's PDF, also known as Version of record

\section{Please check the document version of this publication:}

- A submitted manuscript is the version of the article upon submission and before peer-review. There can be important differences between the submitted version and the official published version of record.

People interested in the research are advised to contact the author for the final version of the publication, or visit the DOI to the publisher's website.

- The final author version and the galley proof are versions of the publication after peer review.

- The final published version features the final layout of the paper including the volume, issue and page numbers.

Link to publication

\footnotetext{
General rights rights.

- You may freely distribute the URL identifying the publication in the public portal. please follow below link for the End User Agreement:

www.umlib.nl/taverne-license

Take down policy

If you believe that this document breaches copyright please contact us at:

repository@maastrichtuniversity.nl

providing details and we will investigate your claim.
}

Copyright and moral rights for the publications made accessible in the public portal are retained by the authors and/or other copyright owners and it is a condition of accessing publications that users recognise and abide by the legal requirements associated with these

- Users may download and print one copy of any publication from the public portal for the purpose of private study or research.

- You may not further distribute the material or use it for any profit-making activity or commercial gain

If the publication is distributed under the terms of Article $25 \mathrm{fa}$ of the Dutch Copyright Act, indicated by the "Taverne" license above, 
Echocardiography modern evaluation of cardiac structure and function 
Copyright B.A. Kaufmann 2016

ISBN 9789461595447

Printing Datawyse | Universitaire Pers Maastricht

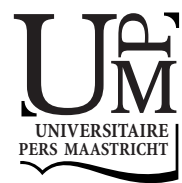




\title{
Echocardiography - modern evaluation of cardiac structure and function
}

\author{
PROEFSCHRIFT \\ ter verkrijging van de graad van doctor aan de Universiteit Maastricht, \\ op gezag van de Rector Magnificus, Prof. dr. L.L.G. Soete \\ volgens het besluit van het College van Decanen, \\ in het openbaar te verdedigen, \\ op vrijdag 11 maart 2016 om 14.00 uur \\ door \\ Beat A. Kaufmann \\ geboren op 17 augustus 1971 te Basel, Zwitserland
}




\section{Promotor}

Prof. dr. H.P. Brunner-La Rocca

\section{Co-promotor}

Dr. V.P.M. van Empel

\section{Beoordelingscommissie}

Prof. dr. H.J.G.M. Crijns (voorzitter)

Dr. S.C.A.M. Bekkers

Dr. C. Knackstedt

Prof. dr. F.W. Prinzen

Prof. dr. J.L. Waltenberger 


\section{Contents}

CHAPTER 1 Introduction

CHAPTER 2 Evidence supportive of impaired myocardial blood flow reserve at high altitude in subjects developing high-altitude pulmonary edema

CHAPTER 3 How reliable are left ventricular ejection fraction cut offs assessed by echocardiography for clinical decision making in patients with heart failure?

CHAPTER 4 Head-to-Head Comparison of Two- and Three-Dimensional Echocardiographic Methods for Left Atrial Chamber Quantification with Magnetic Resonance Imaging

CHAPTER 5 Improvement in left ventricular ejection fraction and reverse remodelling in elderly heart failure patients on intense NT-proBNPguided therapy

CHAPTER 6 Altered left ventricular geometry and torsional mechanics in high altitude-induced pulmonary hypertension: a 3D echocardiographic study

CHAPTER 7 Discussion, Future Directions and Valorisation

Summary/Samenvatting

Acknowledgements

Curriculum vitae

List of Publications 

CHAPTER 1

Introduction 


\section{ECHOCARDIOGRAPHY}

Since the first steps in developing ultrasound as a technique for the non-invasive imaging of the mitral valve in the 1950ties by Dr. I. Edler, and for imaging pericardial effusion in the 1960ties by Dr. H. Feigenbaum, tremendous advances have been made in ultrasound and computer technology ${ }^{1,2}$. What started out as a technique showing an image along a single ultrasound scanline directed at the heart over time (M-Mode) has developed into a technique capable of generating real-time 2-dimensional images of the beating heart ${ }^{3}$. The addition of spectral and colour Doppler ultrasound allowed for nearly complete assessment of the heart and the pulmonary circulation ${ }^{4-11}$,. Another significant step forward was the introduction of transoesophageal echocardiography in $1981^{12}$ which, due to its superior image quality, was soon integrated into clinical cardiology as a valuable adjunct to transthoracic echocardiography. Finally, the development of matrix array ultrasound scanning probes from the 1990ies onwards nowadays allows for real-time three-dimensional imaging of the heart ${ }^{13}$.

Thus, continuous evolvements over the last 6 decades have turned cardiac ultrasound into an imaging technique that is indispensable for the practice of modern cardiology. The research presented in this thesis covers aspects that are all important in modern echocardiography. This encompasses (1) further validation of existing techniques, (2) use of existing techniques in clinical studies testing an intervention, (3) examination of novel tools for the assessment of imaging datasets, and (4) the use of technical advancements in echocardiography to answer specific research questions. Thus, currently employed techniques to measure left ventricular function are examined with regards to their variability and treatment-related changes in a real-world clinical setting. A novel software tool to assess left atrial size on 3-dimensional datasets is validated against a gold standard technique. 3-dimensional strain imaging and myocardial perfusion imaging are used to assess the adaptation of the heart to exposure to high altitude.

\section{ECHOCARDIOGRAPHY FOR THE ASSESSMENT OF MYOCARDIAL PERFUSION}

The coronary arteries, arterioles and the capillary bed all act together in a complex interplay to regulate myocardial perfusion and thus the delivery of nutrients and oxygen to the myocardium ${ }^{14}$. As myocardial perfusion can be disturbed in many clinical scenarios - most notably in the case of coronary artery stenosis - there is a strong interest in the assessment of myocardial perfusion with non-invasive imaging techniques. A precondition for the assessment of myocardial perfusion with echocardiography was the development of a contrast agent that can be detected with ultrasound. Already in the early days of echocardiography it had been noted, that gas bubbles that appear in the 
blood stream for example after intravenous fluid injections, can be detected as bright signals on ultrasound images. From the 1970ies onwards, agitated saline has routinely been used to detect intracardiac shunts. However, these early ultrasound contrast agents had a very short half-life and were too large to pass the pulmonary microcirculation and reach the systemic circulation. With the development of lipid- or albumin encapsulated heavy-weight (perfluorocarbon) gas microbubbles with diameters of around $2-4 \mu \mathrm{m}$ in the 1990ies, opacification of blood in the systemic circulation became possible. Microbubbles have been shown to possess rheologic characteristics very similar to red blood cells, and to remain strictly intravascular ${ }^{15}$, two main pre-requisites for their use for assessing myocardial blood flow. When microbubbles are exposed to a moderate-intensity ultrasound field, they start to resonate, and emit ultrasound waves themselves. These resonated ultrasound waves can be picked up by ultrasound transducer and converted to imaging data, on which the signal brightness corresponds to the number of microbubbles present in the scanplane. With high intensity ultrasound impulses, the microbubbles in the scanplane can be destroyed, and the replenishment can be used to assess myocardial perfusion (reviewed in ${ }^{16}$ ). This technique has been used both in preclinical research ${ }^{17}$ as well as in stable coronary artery disease ${ }^{18}$ and in patients presenting to the emergency department with chest pain ${ }^{19}$ to detect myocardial ischemia. Measurements of myocardial flow reserve correlate well with those from positron emission tomography (PET) ${ }^{20}$.

Exaggerated hypoxic pulmonary vasoconstriction, pulmonary hypertension and high altitude pulmonary edema (HAPE) affect susceptible subjects that ascend rapidly to altitudes $>2500$ meters above sea level ${ }^{21}$. Reduced endothelial function in the pulmonary vasculature owing to a reduced bioavailability of nitric oxide has been implicated in the pathogenesis of HAPE. However, catheterisation data show that the development of HAPE occurs together with a rise in capillary hydrostatic pressure ${ }^{22}$. These data suggest that changes in left heart physiology that lead to an increase in left ventricular filling pressure might also play a role. While the systolic function of the left ventricle has documented to remain normal in subjects with HAPE susceptibility at high altitude, minor impairments in diastolic function have been shown ${ }^{23}$, and systemic endothelial function has been demonstrated to be abnormal ${ }^{24}$. We therefore used myocardial contrast echocardiography to assess whether myocardial flow reserve as a readout of endothelial function is different in subjects with HAPE susceptibility in comparison to normal individuals at high altitude.

\section{ECHOCARDIOGRAPHY FOR THE ASSESSMENT OF CARDIAC CHAMBER SIZE AND FUNCTION}

Left ventricular ejection fraction (LVEF) is without any doubt the parameter most frequently asked for when performing echocardiography. LVEF has prognostic implications 
25 and is frequently used for deciding whether a patient gets a specific therapeutic intervention, be it pharmacological or interventional. Despite the wide availability of methods for measurement of LVEF such as the modified biplane Simpson's method ${ }^{26}$, a visual assessment of LVEF with inherent limitations due to interobserver and intraobserver variability is still often used. In addition, even state-of-the art biplane measurement of LVEF has istits limitations due to image dropout of endocardium that is oriented parallel to the ultrasound beam and apical foreshortening. It is assumed that in the near future, increased use of three-dimensional echocardiography (3DE) will provide more accurate and reproducible measurements of $\operatorname{LVEF}^{27}$. Given the known inaccuracies of LVEF measurements, in the present thesis we first assessed the variability of reallife measurements on an individual patient basis. We then went on to assess the evolution of LVEF over time in heart failure patients with a NT-proBNP guided versus a symptom-guided strategy of intensified treatment.

In addition to measurement of left ventricular (LV) cavity size for the assessment of function, strain imaging has been developed for a standardized quantification of global and regional LV function. Strain imaging measures the deformation of the myocardium over the cardiac cycle. Strain is dimensionless and is expressed as a percentage change of the length of cardiac fibers from end-diastole to end-systole. 2-dimensional echocardiography allows for the measurement of longitudinal, circumferential and radial deformation of the left ventricular myocardium ${ }^{28}$. In addition, ventricular contraction involves a counterclockwise rotation of the base and a clockwise rotation of the apex, resulting in a twisting motion of the heart during systole ${ }^{29}$. 2-dimensional echocardiography has been used for the assessment of LV rotational mechanics ${ }^{28}$. However, 3DE has the distinct advantage that out of plane movement of structures that are being tracked does not occur. It is known that longstanding pulmonary artery hypertension can alter left ventricular geometry and thus left ventricular torsion ${ }^{30}$, however, left ventricular remodeling was a potential confounder in this study. We therefore aimed to study the influence of short-term increases in pulmonary artery pressure on left ventricular torsion in healthy subjects ascending to high altitude, where confounders like left ventricular remodeling can be excluded.

Echocardiography is also routinely used to measure the size of the left atrium (LA). There is a direct relationship between left ventricular filling pressures and LA remodeling ${ }^{31}$, and a left atrial volume index $\geq 34 \mathrm{ml} / \mathrm{m} 2$ has been shown to independently predict death, heart failure, atrial fibrillation and stroke ${ }^{32}$. Biplane 2-dimensional imaging has typically been used to determine LA volume. Dedicated software algorithms have been developed recently to assess left atrial volumes on $3 \mathrm{DE}$, which are thought to be more accurate and reproducible, but have not been extensively validated. We therefore validated the accuracy of a novel software tool (4D LA Analysis, TomTec Imaging Systems) for the assessment of left atrial volumes against the gold standard of magnetic resonance imaging derived values. 


\section{AIMS AND OUTLINE OF THIS THESIS}

The overall aim of this thesis was to use state-of the art echocardiography to improve knowledge of left ventricular and left atrial physiology, structure, and function. In order to achieve this aim, we used different echocardiographic techniques and applications in different patient populations with specific alterations. Thus, we investigated (I) subjects exposed to high altitude as a model of acute pulmonary hypertension and possibly acute endothelial dysfunction (Chapter 2 and 6), (II) patients scheduled to undergo pulmonary vein isolation as a model of different severity of left atrial structural and functional abnormalities (Chapter 4), and (III) a large clinical heart failure trial cohort as a model of left-ventricular systolic dysfunction and dilation (Chapter 3 and 5). We used contrast enhanced ultrasound to assess myocardial blood flow reserve at high altitude in normal subjects compared to subjects developing high altitude pulmonary edema (Chapter 2). The reliability of real-life assessment of LVEF was assessed in a large heart failure trial (Chapter 3). A novel software algorithm for the measurement of left atrial volumes was validated against magnetic resonance imaging (Chapter 4). The influence of BNP-guidance in heart failure treatment on LVEF was assessed over time in a large heart failure trial (Chapter 5). Finally, 3DE of cardiac torsion was used to investigate the influence of isolated pulmonary hypertension occurring at high altitude on left ventricular mechanics (Chapter 6). 


\section{REFERENCES}

1. Edler I, Gustafson A. Ultrasonic cardiogram in mitral stenosis; preliminary communication. Acta Med Scand. 1957;159:85-90

2. Feigenbaum $H$, Waldhausen JA, Hyde LP. Ultrasound diagnosis of pericardial effusion. JAMA. 1965;191:711-714

3. Bom N, Lancee CT, Honkoop J, Hugenholtz PG. Ultrasonic viewer for cross-sectional analyses of moving cardiac structures. Biomed Eng. 1971;6:500-503, 505

4. Holen J, Simonsen S. Determination of pressure gradient in mitral stenosis with doppler echocardiography. Br Heart J. 1979;41:529-535

5. Hatle L, Brubakk A, Tromsdal A, Angelsen B. Noninvasive assessment of pressure drop in mitral stenosis by doppler ultrasound. Br Heart J. 1978;40:131-140

6. Hatle L, Angelsen BA, Tromsdal A. Non-invasive assessment of aortic stenosis by doppler ultrasound. $\mathrm{Br}$ Heart J. 1980;43:284-292

7. Omoto R, Yokote Y, Takamoto S, Kyo S, Ueda K, Asano H, Namekawa K, Kasai C, Kondo Y, Koyano A. The development of real-time two-dimensional doppler echocardiography and its clinical significance in acquired valvular diseases. With special reference to the evaluation of valvular regurgitation. Jpn Heart J. 1984;25:325-340

8. Helmcke F, Nanda NC, Hsiung MC, Soto B, Adey CK, Goyal RG, Gatewood RP, Jr. Color doppler assessment of mitral regurgitation with orthogonal planes. Circulation. 1987;75:175-183

9. Perry GJ, Helmcke F, Nanda NC, Byard C, Soto B. Evaluation of aortic insufficiency by doppler color flow mapping. J Am Coll Cardiol. 1987;9:952-959

10. Chopra HK, Nanda NC, Fan P, Kapur KK, Goyal R, Daruwalla D, Pacifico A. Can two-dimensional echocardiography and doppler color flow mapping identify the need for tricuspid valve repair? J Am Coll Cardiol. 1989;14:1266-1274

11. Enriquez-Sarano M, Miller FA, Jr., Hayes SN, Bailey KR, Tajik AJ, Seward JB. Effective mitral regurgitant orifice area: Clinical use and pitfalls of the proximal isovelocity surface area method. J Am Coll Cardiol. 1995;25:703-709

12. Hanrath $\mathrm{P}$, Kremer $\mathrm{P}$, Langenstein BA, Matsumoto M, Bleifeld W. [transesophageal echocardiography. A new method for dynamic ventricle function analysis]. Dtsch Med Wochenschr. 1981;106:523-525

13. Sheikh K, Smith SW, von Ramm O, Kisslo J. Real-time, three-dimensional echocardiography: Feasibility and initial use. Echocardiography. 1991;8:119-125

14. Jayaweera AR, Wei K, Coggins M, Bin JP, Goodman C, Kaul S. Role of capillaries in determining cbf reserve: New insights using myocardial contrast echocardiography. Am J Physiol. 1999;277:H2363-2372

15. Keller MW, Segal SS, Kaul S, Duling B. The behavior of sonicated albumin microbubbles within the microcirculation: A basis for their use during myocardial contrast echocardiography. Circ Res. 1989;65:458-467

16. Kaufmann BA, Wei K, Lindner JR. Contrast echocardiography. Curr Prob/ Cardiol. 2007;32:51-96

17. Wei K, Jayaweera AR, Firoozan S, Linka A, Skyba DM, Kaul S. Quantification of myocardial blood flow with ultrasound-induced destruction of microbubbles administered as a constant venous infusion. Circulation. 1998;97:473-483

18. Wei K, Ragosta M, Thorpe J, Coggins M, Moos S, Kaul S. Noninvasive quantification of coronary blood flow reserve in humans using myocardial contrast echocardiography. Circulation. 2001;103:2560-2565

19. Rinkevich D, Kaul S, Wang XQ, Tong KL, Belcik T, Kalvaitis S, Lepper W, Dent JM, Wei K. Regional left ventricular perfusion and function in patients presenting to the emergency department with chest pain and no st-segment elevation. Eur Heart J. 2005;26:1606-1611

20. Vogel R, Indermuhle A, Reinhardt J, Meier P, Siegrist PT, Namdar M, Kaufmann PA, Seiler C. The quantification of absolute myocardial perfusion in humans by contrast echocardiography: Algorithm and validation. J Am Coll Cardiol. 2005;45:754-762

21. Bartsch P, Mairbaurl H, Maggiorini M, Swenson ER. Physiological aspects of high-altitude pulmonary edema. J Appl Physiol (1985). 2005;98:1101-1110 
22. Maggiorini M, Melot C, Pierre S, Pfeiffer F, Greve I, Sartori C, Lepori M, Hauser M, Scherrer U, Naeije R. High-altitude pulmonary edema is initially caused by an increase in capillary pressure. Circulation. 2001;103:2078-2083

23. Allemann Y, Rotter M, Hutter D, Lipp E, Sartori C, Scherrer U, Seiler C. Impact of acute hypoxic pulmonary hypertension on Iv diastolic function in healthy mountaineers at high altitude. Am J Physiol Heart Circ Physiol. 2004;286:H856-862

24. Berger MM, Hesse C, Dehnert C, Siedler H, Kleinbongard P, Bardenheuer HJ, Kelm M, Bartsch P, Haefeli WE. Hypoxia impairs systemic endothelial function in individuals prone to high-altitude pulmonary edema. Am J Respir Crit Care Med. 2005;172:763-767

25. White HD, Norris RM, Brown MA, Brandt PW, Whitlock RM, Wild CJ. Left ventricular end-systolic volume as the major determinant of survival after recovery from myocardial infarction. Circulation. 1987;76:4451

26. Lang RM, Bierig M, Devereux RB, Flachskampf FA, Foster E, Pellikka PA, Picard MH, Roman MJ, Seward J, Shanewise JS, Solomon SD, Spencer KT, Sutton MS, Stewart WJ. Recommendations for chamber quantification: A report from the american society of echocardiography's guidelines and standards committee and the chamber quantification writing group, developed in conjunction with the european association of echocardiography, a branch of the european society of cardiology. J Am Soc Echocardiogr. 2005;18:14401463

27. Dorosz JL, Lezotte DC, Weitzenkamp DA, Allen LA, Salcedo EE. Performance of 3-dimensional echocardiography in measuring left ventricular volumes and ejection fraction: A systematic review and metaanalysis. J Am Coll Cardiol. 2012;59:1799-1808

28. Hurlburt HM, Aurigemma GP, Hill JC, Narayanan A, Gaasch WH, Vinch CS, Meyer TE, Tighe DA. Direct ultrasound measurement of longitudinal, circumferential, and radial strain using 2-dimensional strain imaging in normal adults. Echocardiography. 2007;24:723-731

29. Buckberg G, Hoffman JI, Nanda NC, Coghlan C, Saleh S, Athanasuleas C. Ventricular torsion and untwisting: Further insights into mechanics and timing interdependence: A viewpoint. Echocardiography. 2011;28:782-804

30. Puwanant S, Park M, Popovic ZB, Tang WH, Farha S, George D, Sharp J, Puntawangkoon J, Loyd JE, Erzurum SC, Thomas JD. Ventricular geometry, strain, and rotational mechanics in pulmonary hypertension. Circulation. 2010;121:259-266

31. Tsang TS, Barnes ME, Gersh BJ, Bailey KR, Seward JB. Left atrial volume as a morphophysiologic expression of left ventricular diastolic dysfunction and relation to cardiovascular risk burden. Am J Cardiol. 2002;90:1284-1289

32. Abhayaratna WP, Seward JB, Appleton CP, Douglas PS, Oh JK, Tajik AJ, Tsang TS. Left atrial size: Physiologic determinants and clinical applications. J Am Coll Cardiol. 2006;47:2357-2363 



\section{CHAPTER 2}

Evidence supportive of impaired myocardial blood flow reserve at high altitude in subjects developing high-altitude pulmonary edema

Beat A. Kaufmann, Alain M. Bernheim, Stephanie Kiencke, Manuel Fischler, Jiri Sklenar, Heimo Mairbäurl, Marco Maggiorini, Hans-Peter Brunner-La Rocca

AJP - Heart and Circulatory Physiology 2008; 294:1651-1657 


\section{ABSTRACT}

Aims: An exaggerated increase in pulmonary artery pressure is the hallmark of HAPE and is associated with endothelial dysfunction of the pulmonary vasculature. Whether the myocardial circulation is affected by endothelial dysfunction is not known. The aim of this study was therefore to investigate whether myocardial blood flow reserve $(\mathrm{MBFr})$ is altered in mountaineers developing high altitude pulmonary edema (HAPE).

Methods and Results: Healthy mountaineers taking part in a trial of prophylactic treatment of HAPE were examined at low $(490 \mathrm{~m})$ and high altitude $(4559 \mathrm{~m})$. MBFr was derived from low mechanical index contrast echocardiography performed at rest and during submaximal exercise. Among 24 subjects evaluated for MBFr, 9 were HAPEsusceptibles on prophylactic treatment with dexamethasone or tadalafil, 6 were HAPE susceptibles on placebo, and 9 persons whithout HAPE susceptibility served as controls. At low altitude, MBFr did not differ between groups. At high altitude, MBFr increased significantly in HAPE susceptibles on treatment and control persons $(2.1 \pm 0.8$ and $2.9 \pm 0.9$ at low and high altitude, respectively, $p=0.001)$, but not in HAPE susceptibles on placebo $(2.5 \pm 0.3$ and $2.0 \pm 1.3$ at low and high altitude, respectively, $p>0.1)$. The response to high altitude was significantly different between the two groups $(p=0.01)$. There was a significant inverse relation between the increase in the pressure gradient across the tricuspid valve and the change in myocardial blood flow reserve.

Conclusions: HAPE-susceptible individuals not taking prophylactic treatment exhibit a reduced $\mathrm{MBFr}$ compared to either treated HAPE-susceptibles or healthy controls at high altitude. 


\section{INTRODUCTION}

High-altitude pulmonary edema (HAPE) is a potentially fatal condition occurring in nonacclimatized individuals ascending rapidly to altitudes above $2500 \mathrm{~m}$. In an unselected population, approximately 5-6\% develop HAPE usually within 2 to 3 days if ascending rapidly to $4500 \mathrm{~m}$, whereas individuals with a history of HAPE have a $60 \%$ chance of recurrence ${ }^{1}$. The pathophysiological hallmark of HAPE is an exaggerated hypoxic pulmonary vasoconstriction with an abnormal increase in pulmonary artery ${ }^{2-5}$ and capillary pressure ${ }^{6}$. Transarteriolar fluid leakage ${ }^{7,8}$, irregular distribution of vasoconstriction with regional overperfusion, or venoconstriction ${ }^{9}$ have been proposed as mechanisms, while inflammatory mechanisms do not seem to play a major role ${ }^{10}$. Decreased concentrations of nitric oxide (NO) in exhaled air are found in HAPE-susceptibles exposed to hypoxia ${ }^{11}$. Furthermore, if individuals with HAPE inhale NO at high altitude, pulmonary artery pressure (PAP) is lowered and gas exchange improved to values seen in normal individuals ${ }^{12,13}$. These data suggest an impaired endothelial function in HAPEsusceptibles leading to decreased bioavailability of NO and an exaggerated hypoxic vasoconstriction.

Systolic function of the left ventricle is normal in individuals exposed to high altitude, irrespective of HAPE susceptibility. Diastolic dysfunction may be present ${ }^{14,15}$, but data are not uniform and the precise mechanism is unknown. Recently, endothelial dysfunction of the systemic vasculature has been found in HAPE-susceptible subjects when exposed to hypoxia ${ }^{14}$. However, if there is also endothelial dysfunction in the myocardial microcirculation similar to that in the pulmonary vasculature is not known. Furthermore, the effect of HAPE prevention on myocardial blood flow is not known. Therefore, the aim of the study was to investigate if impaired endothelial function is present in the coronary circulation and if medical HAPE prophylaxis might influence myocardial microcirculation. Therefore, we measured myocardial blood flow reserve (MBFr) with myocardial contrast echocardiography at high altitude in HAPE susceptibles participating in a placebo controlled double-blind study for HAPE prevention and in resistant controls. 


\section{METHODS}

\section{Study subjects and design}

A total of 29 mountaineers, 4 women and 25 men, with a history of at least one episode of HAPE (termed HAPE-susceptibles, HAPE-S), and 10 control subjects ( 1 woman) without history of HAPE, were studied. All HAPE-S subjects participated in a study investigating the effect of the phosphodiesterase inhibitor tadalafil (Cialis ${ }^{\circledR}$, Lilly) or dexamethasone (Fortecortin ${ }^{\circledR}$, Merck) for HAPE prevention. Details on study sequence, medication intake, and the results have been published elsewhere ${ }^{16}$. Apart from medication intake, procedures in control subjects did not differ. None of the study subjects had any known cardiovascular risk factor. The study protocol was approved by the ethics committees of the involved institutions and all participants gave written informed consent.

Baseline measurements were performed in Zurich (490m) 2-4 weeks prior to the investigation at the high-altitude research laboratory at Capanna Regina Margherita, Italy $(4559 \mathrm{~m})$. In Zurich, patients underwent clinical examination and, thereafter, bicycle exercise testing until exhaustion to assess the maximal exercise capacity. On the following day, Doppler echocardiography was performed at rest and during exercise with the subject sitting on a bicycle ergometer in semi-recumbent position. Stress echocardiographic data were recorded at a workload corresponding to $40 \%$ of the individual peak exercise capacity.

The subjects ascended within 22 hours from $1130 \mathrm{~m}$ to $4559 \mathrm{~m}$. The subjects ascended by cable car to an altitude of $3200 \mathrm{~m}$, from where they climbed approximately $1 \frac{1}{2}$ hours to $3600 \mathrm{~m}$. After an overnight stay, they climbed within an additional $4 \frac{1}{2}$ hours to $4559 \mathrm{~m}$ altitude. Echocardiography was performed on the following day at rest and during bicycle ergometry at a workload $70 \%$ of that at low altitude (i.e. $28 \%$ of individual peak exercise capacity). Workload was reduced to compensate for the effects of high altitude based on previous experience ${ }^{17}$.

HAPE was clinically suspected at the appearance of dry cough, orthopnea or pulmonary rales. A postero-anterior thorax radiograph was then taken using a mobile unit (TRS, Siemens, Stockholm, Sweden) with a fixed distance of $1.4 \mathrm{~m}$ at $95 \mathrm{kV}$ and 3 to $6 \mathrm{~mA} / \mathrm{s}$. Radiographs were scored retrospectively by a second radiologist blinded to other study results. HAPE was defined as previously reported ${ }^{18}$.

\section{Assessment of myocardial blood flow reserve}

MBFr was assessed using myocardial contrast echocardiography. The ultrasound contrast agent Sonovue ${ }^{\circledR}$ (Bracco, Switzerland) was diluted $1: 5$ in $0.9 \% \mathrm{NaCl}$ and infused intravenously. A constant infusion rate of $50-70 \mathrm{ml} / \mathrm{h}$ was maintained using a prototype mixing pump (Bracco, Switzerland). Contrast images were acquired on a Toshiba Aplio 80 (Toshiba, Japan) equipped with a $4 \mathrm{MHz}$ transducer. The system was set to pulse 
inversion imaging mode at a mechanical index of 0.1 and $40 \mathrm{~dB}$ dynamic range. The compression level and postprocessing algorithms were adjusted for maximum linearity of the imaging. Images were acquired from the apical window. Infusion rate of the contrast agent was optimized to produce a dense, homogeneous left ventricular (LV) cavity opacification with shadowing limited only to the left atrial cavity. The focus of the ultrasound beam was set at the mitral valve level and gain settings were optimized and held constant throughout the examination. Microbubbles in the myocardium were then destroyed by transmitting several high mechanical index (MI of 1.4) frames followed by 10 to 15 seconds of continuous imaging at low mechanical index to observe the contrast replenishment in the LV myocardium. Low and high altitude images at rest and during bicycle ergometer stress were each acquired in a single breathhold. Images were transferred to an off-line computer for image processing using custom software (University of Virginia).

End-systolic frames were selected and aligned manually. The first end-systolic frame after microbubble destruction was then used as a background frame and subtracted from contrast frames. Video-intensities were linearized on a pixel-by-pixel basis by applying an exponential function that was an inverse to the known logarithmic function used by the ultrasound system during the imaging. A region of interest was manually placed on the mid-ventricular septum and linearized data from the region of interest was averaged and fitted to the exponential function:

$$
y=A\left(1-e^{\left(-B_{\bullet} t\right)}\right) \text {, }
$$

where $A$ is proportional to the microvascular myocardial blood volume, $B$ is proportional to the microvascular blood flow velocity, and, hence, the product of $A \bullet B$ is proportional to the microvascular myocardial blood flow $(\mathrm{MBF})^{19}$. The $A$ value measured at the midventricular septum was then normalized by division by a value measured in a region of similar size placed in the adjacent ventricular cavity in order to compensate for possible fluctuations in microbubble concentration during a study ${ }^{20}$. Data on day-to-day variability of this technique have recently been published ${ }^{21}$. MBFr was then calculated as MBF at stress divided by MBF at rest. Investigators analyzing the perfusion data (B.A.K., A.B.) were blinded to study drug intake, HAPE susceptibility, and the subject's clinical outcome. For interobserver variability, the investigators were blinded to the results of the other investigator's analysis.

\section{Doppler Echocardiography}

By continuous wave Doppler, the peak flow velocity of the transtricuspid regurgitant jet was measured for assessment of pressure gradients across the tricuspid valve (dP TR) as an estimate of systolic pulmonary artery pressure. LV diastolic function was assessed in the apical four chamber view. The sample volume of pulsed wave Doppler was placed at the tips of the mitral valve leaflets. The obtained values were peak flow velocity ( $E$, 


\section{CHAPTER 2}

$\mathrm{cm} / \mathrm{s}$ ) and deceleration time (ms) of the early diastolic filling, peak flow velocity (A, $\mathrm{cm} / \mathrm{s}$ ) of the late diastolic filling, and isovolumic relaxation time. Tissue Doppler imaging was used for measuring tissue velocities at the basal septum to obtain the mitral annular motion velocity $\left(E^{\prime}, \mathrm{cm} / \mathrm{s}\right)$ during early diastole.

\section{Statistical analysis}

Statistical analysis was performed with a commercially available program (SPSS version 13.0). Values are reported as mean \pm standard deviation unless otherwise stated. Between group comparisons were done using the Mann-Whitney U-test or Kruskal-Wallis $\mathrm{H}$-test, as appropriate, within group comparison using the Wilcoxon-test. For comparison between groups from low to high altitude, changes were calculated and then compared using the appropriate non-parametric test. A p-value of 0.05 was considered to be statistically significant.

\section{RESULTS}

\section{Study population}

The baseline characteristics of the 24 subjects included in the study are presented in Table 1. Among the 24 subjects, 9 were HAPE-S individuals on prophylactic treatment with dexamethasone or tadalafil, 6 were HAPE-S individuals on placebo, and 9 persons without HAPE susceptibility served as controls. Five of these subjects (21\%) eventually developed HAPE; all of them were known HAPE-S individuals receiving placebo (5 of 6 HAPE-S on placebo developed HAPE). None of the control subjects and none of those either on dexamethasone or tadalafil in this study developed HAPE. 
Table 1. Physiological characteristics of the study population

\begin{tabular}{|c|c|c|c|c|c|c|c|c|}
\hline & \multicolumn{2}{|c|}{$\begin{array}{l}\text { HAPE-S Placebo } \\
(n=6)\end{array}$} & \multicolumn{2}{|c|}{$\begin{array}{l}\text { HAPE-S On Treatmen } \\
(n=9)\end{array}$} & \multicolumn{2}{|c|}{$\begin{array}{l}\text { t Control subjects } \\
(n=9)\end{array}$} & \multicolumn{2}{|c|}{$\begin{array}{l}\text { Significance } \\
\text { between groups }\end{array}$} \\
\hline & $490 m$ & $4559 m$ & $490 m$ & $4559 m$ & $490 m$ & $4559 m$ & $490 m$ & $4559 m$ \\
\hline Age, yr & $39 \pm 7$ & & $45 \pm 10$ & & $32 \pm 4$ & & 0.003 & \\
\hline $\begin{array}{l}\text { Heart rate at rest, } \\
\text { beats/min }\end{array}$ & $63 \pm 10$ & $86 \pm 12$ & $63 \pm 12$ & $72 \pm 18$ & $67 \pm 8$ & $88 \pm 10$ & NS & NS \\
\hline $\begin{array}{l}\text { Heart rate exercise*, } \\
\text { beats/min }\end{array}$ & $123 \pm 15$ & $141 \pm 13$ & $121 \pm 12$ & $123 \pm 12$ & $134 \pm 9$ & $133 \pm 5$ & 0.02 & 0.07 \\
\hline $\begin{array}{l}\text { Systolic blood pressure at } \\
\text { rest, } \mathrm{mmHg}\end{array}$ & $121 \pm 11$ & $132 \pm 18$ & $127 \pm 13$ & $124 \pm 13$ & $113 \pm 8$ & $122 \pm 13$ & 0.05 & NS \\
\hline $\begin{array}{l}\text { Diastolic blood pressure } \\
\text { at rest, } \mathrm{mmHg}\end{array}$ & $78 \pm 7$ & $80 \pm 13$ & $85 \pm 10$ & $73 \pm 10$ & $72 \pm 10$ & $76 \pm 12$ & 0.03 & NS \\
\hline $\begin{array}{l}\text { Systolic blood pressure } \\
\text { exercise, } \mathrm{mmHg}\end{array}$ & $153 \pm 16$ & $158 \pm 21$ & $163 \pm 29$ & $147 \pm 12$ & $147 \pm 18$ & $160 \pm 18$ & NS & NS \\
\hline $\begin{array}{l}\text { Diastolic blood pressure } \\
\text { exercise, } \mathrm{mmHg}\end{array}$ & $95 \pm 14$ & $100 \pm 20$ & $97 \pm 10$ & $86 \pm 13$ & $87 \pm 13$ & $90 \pm 16$ & NS & NS \\
\hline $\begin{array}{l}\text { RPP at rest, } \mathrm{mmHg} x \\
\text { beats/min }(x 1000)\end{array}$ & $8 \pm 1,7$ & $11,5 \pm 2,5$ & $8,2 \pm 2,3$ & $9,3 \pm 2,6$ & $8 \pm 1,5$ & $10,1 \pm 1,4$ & NS & NS \\
\hline $\begin{array}{l}\text { RPP exercise, } \mathrm{mmHg} x \\
\text { beats/min }(x 1000)\end{array}$ & $18 \pm 2,8$ & $21,5 \pm 2,2$ & $19,3 \pm 4,4$ & $18,6 \pm 3$ & $19,5 \pm 1,8$ & $21,4 \pm 2,2$ & NS & 0.04 \\
\hline $\mathrm{O} 2$ saturation at rest, \% & $96 \pm 2$ & $66 \pm 11$ & $96 \pm 3$ & $82 \pm 9$ & $97 \pm 1$ & $81 \pm 4$ & NS & 0.002 \\
\hline O2 saturation exercise, $\%$ & $95 \pm 1$ & $61 \pm 11$ & $96 \pm 2$ & $72 \pm 9$ & $96 \pm 1$ & $72 \pm 8$ & NS & NS \\
\hline $\begin{array}{l}\text { Relative change in } \mathrm{O} 2 \\
\text { saturation, \% }\end{array}$ & & $8 \pm 4$ & & $12 \pm 9$ & & $11 \pm 6$ & & NS \\
\hline Hematocrit, \% & $43 \pm 5$ & $44 \pm 5$ & $42 \pm 2$ & $42 \pm 2$ & $44 \pm 2$ & $45 \pm 2$ & NS & NS \\
\hline
\end{tabular}

\section{Hemodynamic parameters}

Hemodynamic parameters are summarized in Table 1 . Heart rates at rest were not different between groups, both at low and high altitude, whereas control subjects attained a slightly higher heart rate during exercise at low altitude, and at high altitude there was a trend toward a higher heart rate during exercise in HAPE-S on placebo. Control subjects had a slightly lower blood pressure at rest at low altitude, but there were no blood pressure differences between groups at high altitude. The rate pressure product (RPP) showed a difference only during exercise at high altitude, which was due to a lower RPP in subjects with prophylactic treatment, while there was no difference between HAPE-S on placebo and controls. Arterial $\mathrm{O} 2$ saturations were not different between groups at low altitude. At high altitude, $\mathrm{O} 2$ saturations were significantly lower in HAPE-S on placebo compared with the other groups at rest. However, there were no differences in $\mathrm{O} 2$ saturations at high altitude during exercise, nor in the relative change of the $\mathrm{O} 2$ saturation caused by exercise at high altitude. The hematocrit was not differ- 


\section{CHAPTER 2}

ent between the groups at both low and high altitude. HAPE-S were older than control subjects. Therefore, all analyses were repeated after adjustment for age, which did not influence the results (data not shown).

\section{Echocardiographic parameters}

Echocardiographic parameters are summarized in Table 2. The increase in PAP was significantly larger in HAPE-S during low-intensity exercise at low altitude $(P<0.001)$. At high altitude, increase in PAP was significantly larger in untreated HAPE-S. Comparing subjects developing HAPE $(n=5)$ and those not developing HAPE $(n=19)$, the increase in PAP from low to high altitude was particularly evident (change in pressure gradient tricuspid regurgitation: $33 \pm 9$ vs. $15 \pm 9 \mathrm{mmHg}, \mathrm{P}<0.001$ ).

All subjects had a normal LV ejection fraction at rest at low altitude. LV ejection fraction increased significantly with exercise in all groups at low and high altitude, and there were no significant differences between the groups. Values for septal and inferolateral wall thickness were normal and not different between groups at low and high altitude. All assessed parameters of diastolic function (E velocity, A velocity, E/A ratio, E/E? ratio, deceleration time, isovolumic relaxation time) were normal at rest at low altitude and did not differ significantly between the groups, despite differences in age.

Table 2. Echocardiographic parameters of the study population

\begin{tabular}{|c|c|c|c|c|c|c|c|c|}
\hline & \multicolumn{2}{|c|}{$\begin{array}{l}\text { HAPE-S Placebo } \\
(n=6)\end{array}$} & \multicolumn{2}{|c|}{$\begin{array}{l}\text { HAPE-S On Treatment } \\
(n=9)\end{array}$} & \multicolumn{2}{|c|}{$\begin{array}{l}\text { Control subjects } \\
(n=9)\end{array}$} & \multicolumn{2}{|c|}{$\begin{array}{l}\text { Significance } \\
\text { between groups }\end{array}$} \\
\hline & $490 m$ & $4559 m$ & $490 m$ & $4559 m$ & $490 m$ & $4559 m$ & $490 m$ & $4559 m$ \\
\hline LVEF at rest, \% & $62 \pm 4$ & $65 \pm 5$ & $64 \pm 6$ & $64 \pm 5$ & $62 \pm 3$ & $65 \pm 9$ & NS & NS \\
\hline LVEF exercise, \% & $70 \pm 2$ & $74 \pm 10$ & $70 \pm 6$ & $76 \pm 6$ & $68 \pm 5$ & $74 \pm 6$ & NS & NS \\
\hline Septum thickness, mm & $10 \pm 1$ & $9 \pm 2$ & $10 \pm 2$ & $10 \pm 1$ & $10 \pm 1$ & $9 \pm 1$ & NS & NS \\
\hline $\begin{array}{l}\text { Inferolateral wall } \\
\text { thickness, mm }\end{array}$ & $9 \pm 1$ & $9 \pm 1$ & $10 \pm 1$ & $9 \pm 1$ & $9 \pm 1$ & $9 \pm 1$ & NS & NS \\
\hline $\mathrm{dp}$ TR at rest*, mmHg & $22 \pm 4$ & $53 \pm 11$ & $19 \pm 7$ & $31 \pm 8$ & $17 \pm 3$ & $33 \pm 6$ & NS & $<0.001$ \\
\hline dp TR exercise, mmHg & $47 \pm 9$ & $74 \pm 20$ & $45 \pm 10$ & $46 \pm 7$ & $28 \pm 3$ & $48 \pm 10$ & $<0.001$ & 0.001 \\
\hline E wave, $\mathrm{cm} / \mathrm{s}$ & $80 \pm 12$ & $78 \pm 22$ & $77 \pm 14$ & $78 \pm 11$ & $71 \pm 15$ & $72 \pm 15$ & NS & NS \\
\hline A wave, $\mathrm{cm} / \mathrm{s}$ & $50 \pm 14$ & $60 \pm 15$ & $56 \pm 15$ & $62 \pm 18$ & $42 \pm 8$ & $52 \pm 11$ & NS & NS \\
\hline E/A ratio & $1.7 \pm 0.4$ & $1.3 \pm 0.4$ & $1.4 \pm 0.3$ & $1.3 \pm 0.3$ & $1.6 \pm 0.4$ & $1.4 \pm 0.3$ & NS & NS \\
\hline Deceleration time, ms & $192 \pm 29$ & $196 \pm 40$ & $191 \pm 47$ & $215 \pm 43$ & $187 \pm 38$ & $194 \pm 24$ & NS & NS \\
\hline IVRT, ms & $86 \pm 17$ & $83 \pm 14$ & $92 \pm 13$ & $95 \pm 16$ & $86 \pm 13$ & $82 \pm 12$ & NS & NS \\
\hline $\mathrm{E} / \mathrm{E}^{\prime}$ ratio & $8.4 \pm 1.7$ & $9.0 \pm 2.8$ & $10.5 \pm 3.2$ & $10.1 \pm 4.5$ & $8.1 \pm 2.1$ & $8.4 \pm 2.4$ & NS & NS \\
\hline
\end{tabular}

Values are means $\pm S D ; n$, no. of subjects. LVEF, left-ventricular ejection fraction; $d p$ TR, pressure gradient tricuspid regurgitation; $E$, peak flow velocity of early diastolic filling; $E^{\prime}$, mitral annular motion velocity; $A$, peak flow velocity of late diastolic filling; IVRT, isovolumic relaxation time. *Response to high altitude different in HAPE-S on placebo vs. other groups: $P<0.05$. 


\section{Myocardial blood flow reserve}

Examples of destruction replenishment sequences and the corresponding curves at high altitude are shown in Fig. 1.
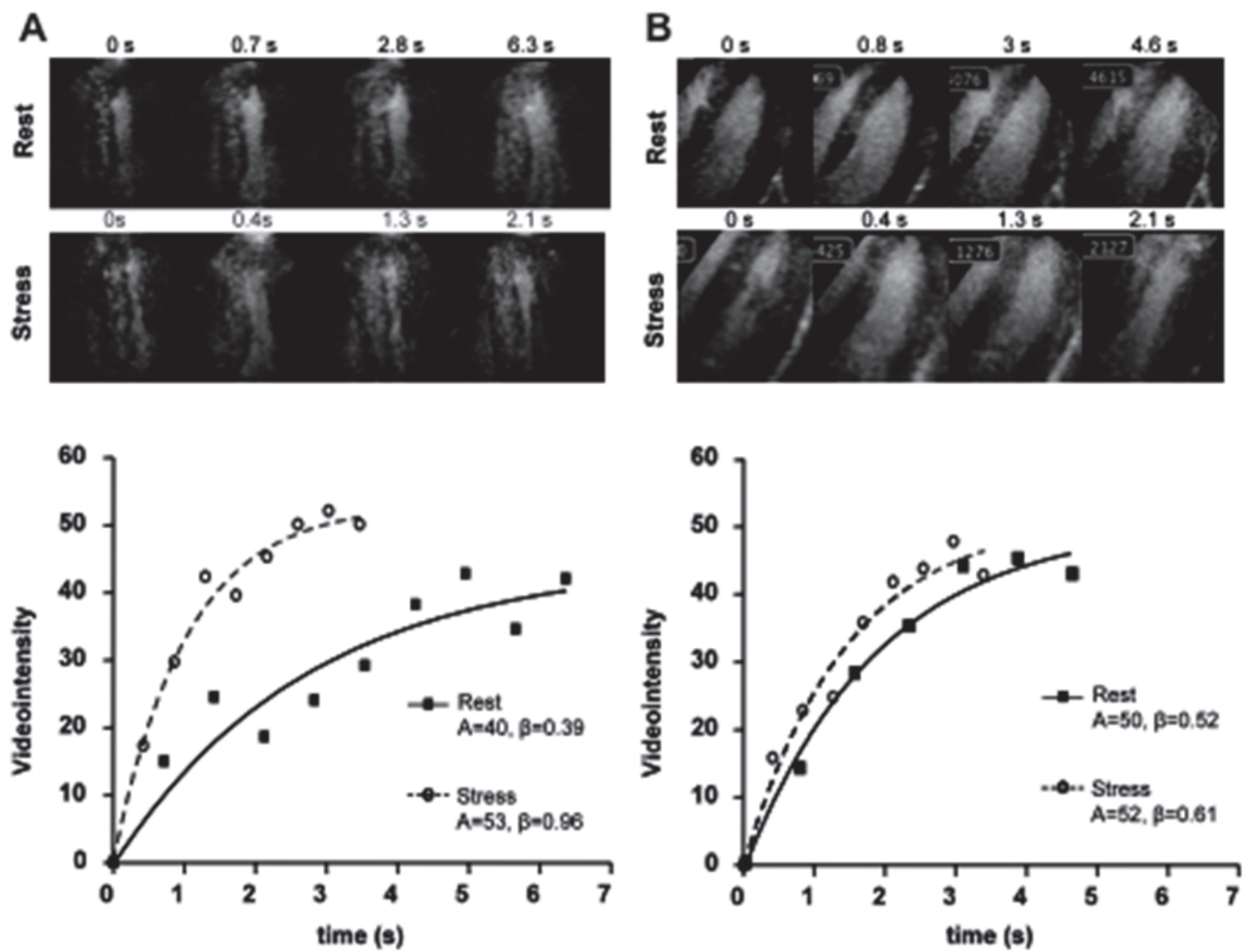

Fig. 1. Examples of destruction replenishment image sequences and the corresponding video-intensity vs. pulsing interval curves from a region of interest drawn in the midventricular septum in one healthy control (A) and one subject developing high-altitude pulmonary edema (HAPE) (B). S, Second-s after microbubble destruction: A, myocardial blood volume: $\beta$, myocardial blood flow velocity.

$A, \square \beta$, and $A \bullet \beta$ values from which MBFr were derived are shown in Table 3. At low altitude, there were no significant differences between the groups regarding $\mathrm{MBFr}$ $(P>0.1$, Fig. 2). At high altitude, MBFr increased significantly $(P<0.05)$ in control subjects and HAPE-S on treatment, whereas it remained unchanged in HAPE-S on placebo. The change in MBFr from low to high altitude significantly differed between HAPE-S on placebo and the other two groups $(P<0.05)$. The subjects ultimately developing HAPE showed no change in $\operatorname{MBFr}(2.5 \pm 0.3$ to $2.0 \pm 1.3, P>0.1$ ) at high altitude, whereas those not developing HAPE showed a highly significant increase ( $2.1 \pm 0.8$ to $2.9 \pm 0.9, P<0.001)$. The response to high altitude significantly differed between the two groups $(P<0.01)$. No differences in the response to high altitude were observed between the two treat- 
ment groups and the control groups (controls $1.9 \pm 0.8$ to $2.8 \pm 1.0$; HAPE-S on tadalafil 2.0 \pm 0.2 to 2.6 \pm 0.8 ; and HAPE-S on dexamethasone $2.4 \pm 1.0$ to 3.1 \pm 1.1 ).

Interobserver variability for determining MBFr was $21 \pm 16 \%$. Importantly, the effects on MBFr in the different groups were observed by both investigators. In particular, results presented did not differ between the two investigators.

There was a significant negative correlation between the increase in PAP and the change in the MBFr from low to high altitude (Spearman $r=-0.48, P=0.02$; Fig. 3). In particular, subjects developing HAPE showed a larger increase in PAP and a decrease in MBFr. Normalized $A \bullet \beta$ at rest at high altitude was not significantly different between groups (8.0 \pm 8.1 in HAPE-S on placebo, $5.3 \pm 3.1$ in HAPE-S on treatment, and $4.0 \pm 1.4$ in controls, $\mathrm{P}=0.4)$.

Table 3. $A, \beta$ and $A \bullet \beta$ values of the study population at rest and during exercise

\begin{tabular}{|c|c|c|c|c|c|c|}
\hline & \multicolumn{2}{|c|}{ HAPE-S Placebo $(n=6)$} & \multicolumn{2}{|c|}{ HAPE-S On Treatment $(n=9)$} & \multicolumn{2}{|c|}{ Control subjects $(n=9)$} \\
\hline & $490 m$ & $4559 m$ & $490 m$ & $4559 m$ & $490 m$ & $4559 m$ \\
\hline$A$ rest & $21 \pm 13$ & $18 \pm 14$ & $19 \pm 13$ & $17 \pm 12$ & $20 \pm 15$ & $16 \pm 6$ \\
\hline$\beta$ rest & $0.30 \pm 0.06$ & $0.38 \pm 0.11$ & $0.30 \pm 0.11$ & $0.32 \pm 0.11$ & $0.30 \pm 0.15$ & $0.24 \pm 0.09$ \\
\hline$A \bullet \beta$ & $5.6 \pm 2.3$ & $8.0 \pm 8.1$ & $5.3 \pm 3.0$ & $5.0 \pm 3.1$ & $5.0 \pm 3.4$ & $4.0 \pm 1.4$ \\
\hline A exercise & $30 \pm 13$ & $29 \pm 22$ & $25 \pm 13$ & $29 \pm 13$ & $19 \pm 9$ & $21 \pm 18$ \\
\hline$\beta$ exercise & $0.48 \pm 0.14$ & $0.48 \pm 0.15$ & $0.52 \pm 0.27$ & $0.47 \pm 0.15$ & $0.48 \pm 0.35$ & $0.56 \pm 0.22$ \\
\hline$A \bullet \beta$ exercise & $13.9 \pm 5.5$ & $12.4 \pm 8.2$ & $12.0 \pm 9.2$ & $13.1 \pm 5.9$ & $8.7 \pm 5.3$ & $10.5 \pm 6.9$ \\
\hline
\end{tabular}

Values are means SD; $\mathrm{n}$, no. Of subjects. $A$, myocardial blood volume; $\beta$, myocardial blood flow velocity; $A \bullet \beta$ myocardial blood flow.

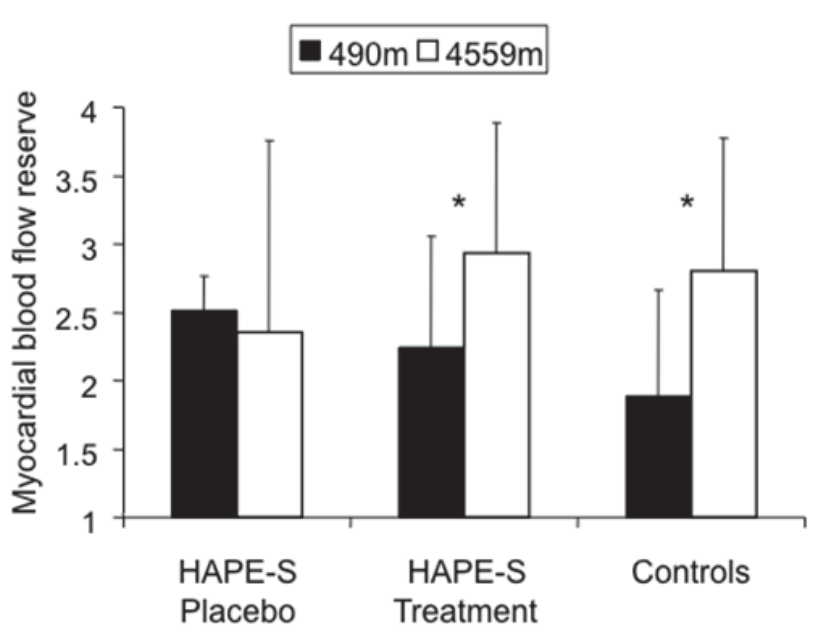

Fig. 2. Myocardial blood flow reserve (MBFr) in HAPE-susceptible (HAPE-S) subjects on placebo, HAPE-S on treatment, and control subjects, showing a significant increase in the MBFr in the latter two groups, but no change in the first. Values are means \pm SD. *significant increase in MBFr at $4.559 \mathrm{~m}$ compared with $490 \mathrm{~m}$, $\mathrm{P}<0.05$. 


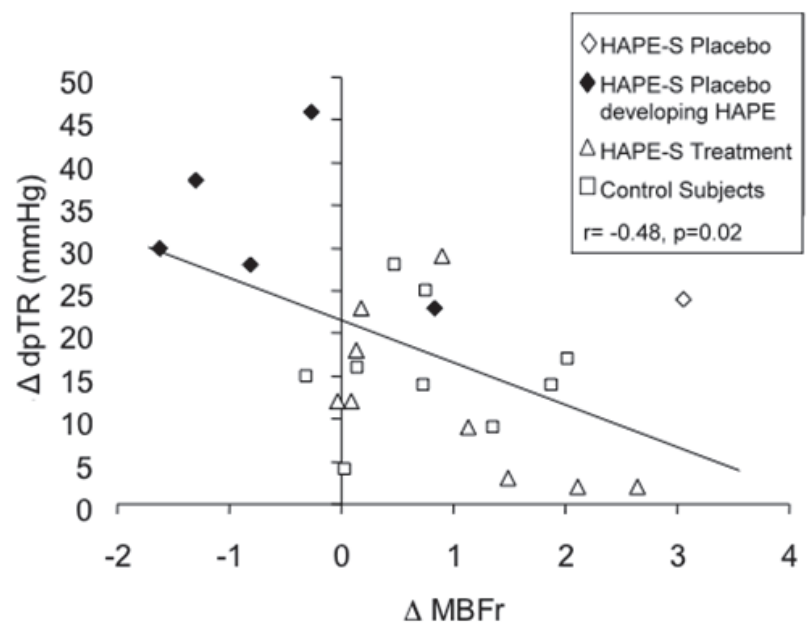

Fig. 3. Correlation between changes $(\Delta)$ from low to high altitude in pressure gradient tricuspid regurgitation $(\mathrm{dP} T \mathrm{R})$ and $\mathrm{MBFr}$ in the three groups. $\bullet$ Subjects ultimately developing HAPE.

\section{Myocardial blood volume and flow velocity reserve}

A reserve and $\beta$ reserve did not differ significantly between the groups at low altitude. At high altitude, $A$ reserve did not change in any group compared to low altitude. Also, ultimate development of HAPE did not have an influence on $A$ reserve (Figure 4). The $\beta$ reserve did not change $(\Delta)$ from low to high altitude in control subjects and HAPE-S on treatment $(\Delta 0.2 \pm 1.7)$, but tended to decrease in HAPE-S on placebo $(\Delta-0.4 \pm 1.2)$. This was particularly evident in those ultimately developing HAPE, compared with those not developing HAPE, in whom $\beta$ reserve was lower (Figure 4). The difference in $\beta$ reserve at high altitude between these two groups was of borderline statistical significance ( $p=0.053)$.

\section{$\rightarrow$ HAPE $\cdot \square \cdot$ No HAPE}
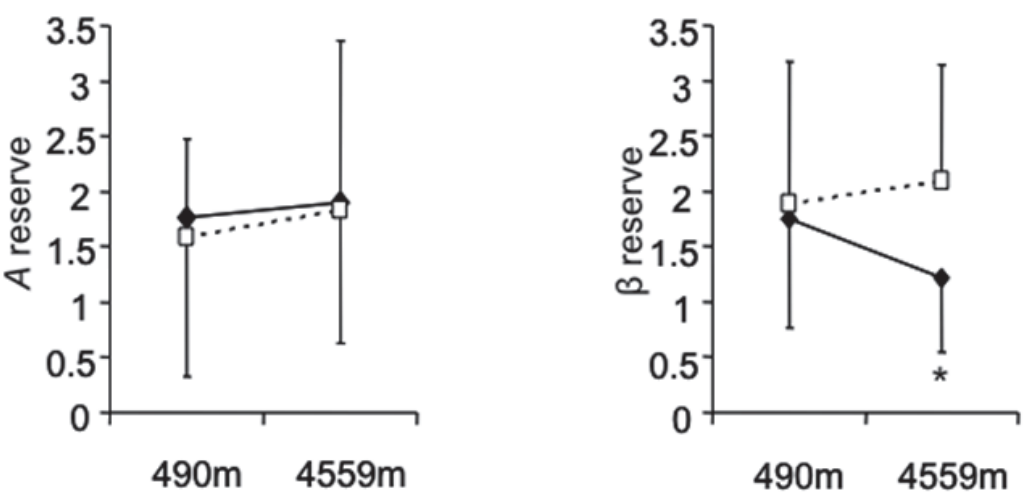

Fig.4. A reserve (left) and $\beta$ reserve (right) in subjects not developing HAPE vs. subjects developing HAPE. Whereas $A$ reserve did not change in either group, $\beta$ reserve showed a decrease in subjects developing HAPE. Values are means $\pm C D$. ${ }^{*} \beta$ reserve at high altitude. $P=0.053$ between the two groups. 


\section{CHAPTER 2}

\section{DISCUSSION}

The main finding of the present study is that HAPE-susceptible individuals on placebo exhibit a reduced MBFr compared to either treated HAPE-susceptibles or healthy controls at high altitude.

MBF during rest and exercise is regulated by mechanical and metabolic vasodilatory factors. Important mechanical factors are the pressure gradient and the resistance across the myocardial microvasculature. The resistance is determined by the anatomy of the coronary vasculature and by blood viscosity ${ }^{22}$. There are a number of factors thought to be involved in local control of MBF, including adenosine, ATP-sensitive potassium channels, and $\mathrm{NO}^{23-25}$. In addition, sympathetic nervous system activation during exercise has been shown to contribute to exercise vasodilation, independent of its effect on local vasodilators ${ }^{26}$.

Myocardial edema caused by pulmonary hypertension has been implicated in LV dysfunction in experimental studies ${ }^{27}$. In our study, the myocardial thickness was not different at low or high altitude and between groups, and thus there was no evidence of myocardial edema as a cause of reduced flow reserve. Also, while blood pressure at rest at low altitude was slightly lower in control subjects, there were no differences in blood pressure during exercise at low altitude and during rest and exercise at high altitude. Right atrial pressures were not measured in this trial. However, we did not find Dopplerechocardiographic evidence (e.g., reduced variability of vena cava inferior during the breathing cycle) for increased right atrial pressure (data not shown), which is compatible with previous invasive data ${ }^{28}$. It seems, therefore, unlikely that a reduction in coronary driving pressure was responsible for the reduced MBFr in HAPE-S on placebo. Similarly, there were no significant differences in hematocrit between groups, arguing against differences in blood viscosity between groups as an explanation for the differences in $\mathrm{MBFr}^{29}$.

An important consideration when comparing blood flow reserve between different subjects is the metabolic demand at rest and during exercise. The RPP as an indicator of cardiac metabolic demand was comparable between groups, both at rest and during exercise at low and high altitude. There was only a small difference during exercise at high altitude, owing to a lower RPP in HAPE-S on prophylactic treatment, whereas the response in HAPE-S and controls was similar. Of note, the RPP during exercise at high altitude were not significantly different from exercise values at low altitude, indicating that reducing the exercise workload at high altitude to $28 \%$ of the maximally attained workload (in Watts) as opposed to the exercise level of $40 \%$ of maximum used at low altitude was indeed justified.

Oxygen saturation significantly differed between groups at rest at high altitude. Given the almost complete oxygen extraction in the myocardium, this could cause differences in resting blood flow between groups. However, when comparing normalized $A \bullet \beta$ at rest at high altitude, there were no statistically significant differences, albeit 
with a trend to higher values for HAPE-S on placebo. In addition, both oxygen saturation values during exercise and the relative changes from rest to exercise did not significantly differ between groups. Thus, while we cannot exclude some contribution of higher blood flow at rest in HAPE-S on placebo to the reduced MBFr in this group, our data indicate that this does not fully explain the differences we found in MBFr.

In our study, we used MCE to assess MBFr for two reasons. First, MCE is the only known technique capable of rendering data on MBFr in a bedside manner, and thus the only technique that can reasonably be applied at high altitude. Second, MCE has the unique property to yield data not only on MBFr, but also on its individual components, myocardial blood volume, and blood flow velocity. In the normal myocardial microcirculation, the increase in blood flow during exercise is governed mainly by a decrease in resistance in the resistance arterioles and the capillary bed ${ }^{30,31}$. Capillary resistance can only decrease through recruitment of previously nonperfused capillaries during exercise on response to increased cellular oxygen requirements. In MCE, changes in the number of functioning capillaries will translate into a change in the A value representing myocardial blood volume. Conversely, the resistance arterioles rely on an intact endothelial and smooth muscle function to lower resistance. Decreasing the resistance in arterioles results in an increase in MBF velocity represented by the $\beta$ value in MCE. Our results indicate that the $A$ reserve is not reduced in HAPE-S individuals on placebo developing pulmonary edema. Therefore, capillary recruitment during exercise does not seem to be affected in these individuals. In contrast, reduced $\beta$ reserve was the main trigger for reduced MBFr in HAPE-S individuals on placebo, particularly those developing HAPE. Thus the reduced MBFr appears to be caused by a lesser decrease in arteriolar resistance rather than a lack of capillary recruitment during exercise, suggesting a reduced vasodilatory capacity in the coronary microcirculation.

Several studies have suggested a reduced availability of NO as the underlying mechanism for the exaggerated pulmonary vasoconstriction in response to hypoxia in HAPE-S individuals $8,10,32$. However, studies examining the physiological control of coronary blood flow during exercise performed in animals have failed to establish a clear role for NO in exercise induced coronary vasodilation ${ }^{33}$. In fact, the exact mechanism leading to an increase in blood flow in the myocardial circulation during exercise remains largely unknown. However, the mechanisms for microcirculatory blood flow regulation at high altitude may well differ from animal studies during normoxia used for the definition of the role of NO in coronary vasodilation. Additionally, in our study, HAPE-S treated with either dexamethasone or the phosphodiesterase inhibitor tadalafil, agents known to influence either the production of NO in the pulmonary vasculature (dexamethasone), or to amplify the effect of NO by inhibiting the breakdown of cGMP (tadalafil) ${ }^{34,35}$ prevented the decrease in MBFr in the untreated HAPE-S. It is thus possible that, in the specific pathological situation of HAPE, a decreased availability of NO in the myocardium may limit MBFr, but further studies are needed to provide definitive evidence for this concept. 


\section{CHAPTER 2}

MBFr at high altitude has not been measured in normal individuals before, but there is one study using positron emission tomography to determine MBFr in normal subjects during hypoxia, corresponding to an altitude of 4,500 $\mathrm{m}$ for ? $1 \mathrm{~h}$. In that study, similar to our results, an increase in MBFr was noted during hypoxia ${ }^{17}$. The fact that this is not the case in HAPE-S individuals on placebo is in line with a recent study ${ }^{14}$ showing reduced endothelial function in the systemic circulation in HAPE-S individuals by measuring forearm blood flow in response to acetylcholine during $4 \mathrm{~h}$ of hypoxia. The present study extends the findings of that study to a physiological setting, where the increase in blood flow is not produced by a pharmacological intervention, but by exercise at high altitude. Furthermore, we show that reduced vasodilator capacity may be present not just acutely, but also after exposure to high altitude for $24 \mathrm{~h}$.

Diastolic LV function has been examined at high altitude in HAPE-S and HAPEresistant subjects in two studies ${ }^{2,36}$. Some changes in diastolic function with an increase in atrial contraction were observed, leading to a new concept of compensated diastolic dysfunction. However, as previously reported, we found no correlation between changes in diastolic function and changes in PAPs from low to high altitude, implying that the two entities may be largely unrelated ${ }^{36}$. In addition, we found no relationship between alterations in MBFr and LV diastolic function at rest. However, further studies should determine whether a hemodynamically relevant diastolic dysfunction may occur during exercise in a subset of mountaineers actually developing HAPE and a reduced MBFr.

Some limitations have to be taken into consideration when interpreting this study. First, all HAPE-S study subjects were taking part in a trial testing whether the phosphodiesterase- 5 inhibitor tadalafil, a selective pulmonary vasodilator, and dexamethasone prevent $\mathrm{HAPE}^{16}$. Both drugs effectively reduced the incidence of HAPE, and thus a relatively small number of subjects eventually developing HAPE were included in this study. Nevertheless, we found a significant effect of altitude and subsequent development of HAPE on MBFr. Importantly, prevention of HAPE by these drugs also prevented reduction in MBFr, with no differences between the two drugs. Second, we cannot exclude an influence of the altered hemodynamics at high altitude on MBFr. However, the fact that an increase in MBFr at high altitude was seen in those not developing HAPE, despite an increase in PAP, argues against altered hemodynamics as the main reason for our findings. Third, the exercise workload at high altitude was reduced by $30 \%$ with respect to the workload at low altitude to account for an expected reduced maximal exercise capacity, which was based on previous findings. A separately conducted maximal exercise test showed that this assumption was adequate (maximal workload $258 \pm 60 \mathrm{~W}$ at low altitude vs. $180 \pm 46 \mathrm{~W}$ at high altitude). Finally, a considerable number of subjects were excluded from analysis due to insufficient image quality. The decision to exclude subjects from the analysis was taken by the two investigators blinded to all other data, and thus the introduction of a bias in the study population seems unlikely. 
However, it should be noted that the excluded subjects were, on average, slightly heavier, with higher body mass index, than the subjects ultimately included in the study.

In conclusion, our data indicate that HAPE-S individuals on placebo show reduced exercise-induced MBFr compared with normal individuals when exposed to hypoxia at high altitude. This reduction may be prevented by treatment with either dexamethasone or sildenafil. Because HAPE susceptibility is relatively common in the general population, these findings might have implications, not only for subjects exposed to high altitude, but also in those with other causes of hypoxia. 


\section{CHAPTER 2}

\section{REFERENCES}

1. Bartsch P, Maggiorini M, Mairbaurl H, Vock P, Swenson ER. Pulmonary extravascular fluid accumulation in climbers. Lancet. 2002;360:571; author reply 571-572

2. Allemann Y, Sartori C, Lepori M, Pierre S, Melot C, Naeije R, Scherrer U, Maggiorini M. Echocardiographic and invasive measurements of pulmonary artery pressure correlate closely at high altitude. Am J Physiol Heart Circ Physiol. 2000;279:H2013-2016

3. Hultgren HN, Lopez CE, Lundberg E, Miller H. Physiologic studies of pulmonary edema at high altitude. Circulation. 1964;29:393-408

4. Penaloza D, Sime F. Circulatory dynamics during high altitude pulmonary edema. Am J Cardiol. 1969;23:369-378

5. Roy SB, Guleria JS, Khanna PK, Manchanda SC, Pande JN, Subba PS. Haemodynamic studies in high altitude pulmonary oedema. Br Heart J. 1969;31:52-58

6. Maggiorini M, Melot C, Pierre S, Pfeiffer F, Greve I, Sartori C, Lepori M, Hauser M, Scherrer U, Naeije R. High-altitude pulmonary edema is initially caused by an increase in capillary pressure. Circulation. 2001;103:2078-2083

7. Schoene RB, Swenson ER, Pizzo CJ, Hackett PH, Roach RC, Mills WJ, Jr., Henderson WR, Jr., Martin TR. The lung at high altitude: Bronchoalveolar lavage in acute mountain sickness and pulmonary edema. J Appl Physiol (1985). 1988;64:2605-2613

8. Swenson ER, Maggiorini M, Mongovin S, Gibbs JS, Greve I, Mairbaurl H, Bartsch P. Pathogenesis of highaltitude pulmonary edema: Inflammation is not an etiologic factor. JAMA. 2002;287:2228-2235

9. Zhao Y, Packer CS, Rhoades RA. Pulmonary vein contracts in response to hypoxia. Am J Physiol. 1993;265:L87-92

10. Busch T, Bartsch P, Pappert D, Grunig E, Hildebrandt W, Elser H, Falke KJ, Swenson ER. Hypoxia decreases exhaled nitric oxide in mountaineers susceptible to high-altitude pulmonary edema. Am J Respir Crit Care Med. 2001;163:368-373

11. Anand IS, Prasad BA, Chugh SS, Rao KR, Cornfield DN, Milla CE, Singh N, Singh S, Selvamurthy W. Effects of inhaled nitric oxide and oxygen in high-altitude pulmonary edema. Circulation. 1998;98:2441-2445

12. Allemann Y, Rotter M, Hutter D, Lipp E, Sartori C, Scherrer U, Seiler C. Impact of acute hypoxic pulmonary hypertension on Iv diastolic function in healthy mountaineers at high altitude. Am J Physiol Heart Circ Physiol. 2004;286:H856-862

13. Scherrer U, Vollenweider L, Delabays A, Savcic M, Eichenberger U, Kleger GR, Fikrle A, Ballmer PE, Nicod P, Bartsch P. Inhaled nitric oxide for high-altitude pulmonary edema. N Engl J Med. 1996;334:624-629

14. Berger MM, Hesse C, Dehnert C, Siedler H, Kleinbongard P, Bardenheuer HJ, Kelm M, Bartsch P, Haefeli WE. Hypoxia impairs systemic endothelial function in individuals prone to high-altitude pulmonary edema. Am J Respir Crit Care Med. 2005;172:763-767

15. Kjaergaard J, Snyder EM, Hassager C, Olson TP, Oh JK, Johnson BD. The effect of $18 \mathrm{~h}$ of simulated high altitude on left ventricular function. Eur J Appl Physiol. 2006;98:411-418

16. Maggiorini M, Brunner-La Rocca HP, Peth S, Fischler M, Bohm T, Bernheim A, Kiencke S, Bloch KE, Dehnert C, Naeije R, Lehmann T, Bartsch P, Mairbaurl H. Both tadalafil and dexamethasone may reduce the incidence of high-altitude pulmonary edema: A randomized trial. Ann Intern Med. 2006;145:497-506

17. Wyss CA, Koepfli P, Fretz G, Seebauer M, Schirlo C, Kaufmann PA. Influence of altitude exposure on coronary flow reserve. Circulation. 2003;108:1202-1207

18. Vock P, Fretz C, Franciolli M, Bartsch P. High-altitude pulmonary edema: Findings at high-altitude chest radiography and physical examination. Radiology. 1989;170:661-666

19. Wei K, Jayaweera AR, Firoozan S, Linka A, Skyba DM, Kaul S. Quantification of myocardial blood flow with ultrasound-induced destruction of microbubbles administered as a constant venous infusion. Circulation. 1998;97:473-483 


\section{EVIDENCE SUPPORTIVE OF IMPAIRED MYOCARDIAL BLOOD FLOW}

20. Yamada S, Komuro K, Mikami T, Kudo N, Onozuka H, Goto K, Fujii S, Yamamoto K, Kitabatake A. Novel quantitative assessment of myocardial perfusion by harmonic power doppler imaging during myocardial contrast echocardiography. Heart. 2005;91:183-188

21. Daut J, Maier-Rudolph W, von Beckerath N, Mehrke G, Gunther K, Goedel-Meinen L. Hypoxic dilation of coronary arteries is mediated by atp-sensitive potassium channels. Science. 1990;247:1341-1344

22. Grunig E, Mereles D, Hildebrandt W, Swenson ER, Kubler W, Kuecherer H, Bartsch P. Stress doppler echocardiography for identification of susceptibility to high altitude pulmonary edema. J Am Coll Cardiol. 2000;35:980-987

23. Archie JP, Jr. Mechanical determinants of myocardial blood flow and its distribution. Ann Thorac Surg. 1975;20:39-45

24. Berne RM. The role of adenosine in the regulation of coronary blood flow. Circ Res. 1980;47:807-813

25. Furchgott RF, Zawadzki JV. The obligatory role of endothelial cells in the relaxation of arterial smooth muscle by acetylcholine. Nature. 1980;288:373-376

26. Tune JD, Richmond KN, Gorman MW, Feigl EO. Control of coronary blood flow during exercise. Exp Biol Med (Maywood). 2002;227:238-250

27. Davis KL, Mehlhorn U, Laine GA, Allen SJ. Myocardial edema, left ventricular function, and pulmonary hypertension. J Appl Physiol (1985). 1995;78:132-137

28. Kawashima A, Kubo K, Kobayashi T, Sekiguchi M. Hemodynamic responses to acute hypoxia, hypobaria, and exercise in subjects susceptible to high-altitude pulmonary edema. J Appl Physiol (1985). 1989;67:1982-1989

29. Reinhart WH. Molecular biology and self-regulatory mechanisms of blood viscosity: A review. Biorheology. 2001;38:203-212

30. Chilian WM, Eastham CL, Marcus ML. Microvascular distribution of coronary vascular resistance in beating left ventricle. Am J Physiol. 1986;251:H779-788

31. Jayaweera AR, Wei K, Coggins M, Bin JP, Goodman C, Kaul S. Role of capillaries in determining cbf reserve: New insights using myocardial contrast echocardiography. Am J Physiol. 1999;277:H2363-2372

32. Duplain H, Sartori C, Lepori M, Egli M, Allemann Y, Nicod P, Scherrer U. Exhaled nitric oxide in highaltitude pulmonary edema: Role in the regulation of pulmonary vascular tone and evidence for a role against inflammation. Am J Respir Crit Care Med. 2000;162:221-224

33. Tune JD, Richmond KN, Gorman MW, Feigl EO. Role of nitric oxide and adenosine in control of coronary blood flow in exercising dogs. Circulation. 2000;101:2942-2948

34. Murata T, Hori M, Sakamoto K, Karaki H, Ozaki H. Dexamethasone blocks hypoxia-induced endothelial dysfunction in organ-cultured pulmonary arteries. Am J Respir Crit Care Med. 2004;170:647-655

35. Zhao L, Mason NA, Morrell NW, Kojonazarov B, Sadykov A, Maripov A, Mirrakhimov MM, Aldashev A, Wilkins MR. Sildenafil inhibits hypoxia-induced pulmonary hypertension. Circulation. 2001;104:424-428

36. Bernheim AM, Kiencke S, Fischler M, Dorschner L, Debrunner J, Mairbaurl H, Maggiorini M, Brunner-La Rocca HP. Acute changes in pulmonary artery pressures due to exercise and exposure to high altitude do not cause left ventricular diastolic dysfunction. Chest. 2007;132:380-387 



\section{CHAPTER 3}

How reliable are left ventricular ejection fraction cut offs assessed by echocardiography for clinical decision making in patients with heart failure?

Beat A. Kaufmann, Son Y. Min, Kaatje Goetschalckx, Alain M. Bernheim, Peter T. Buser, Matthias E. Pfisterer, Hans-Peter Brunner-La Rocca

Int J Cardiovasc Imaging 2013; 29: 581-588 


\section{CHAPTER 3}

\section{ABSTRACT}

Aims: To study the potential influence of the variability in the assessment of echocardiographically measured left ventricular ejection fraction (LVEF) on indications for the implantation of internal cardioverter defibrillator and/or cardiac resynchronization devices in heart failure patients.

Methods and results: TIME-CHF was a multicenter trial comparing NT-BNP versus symptom-guided therapy in patients aged $\geq 60$ years. Patients had their LVEF assessed at the recruiting centre using visual assessment, the area-length or biplane Simpson's method. Echocardiographic data were transferred to the study core-lab for re-assessment. Reassessment in the core-lab was done with biplane Simpson's method, and included an appraisal of image quality. 413 patients had the LVEF analyzed at the recruiting centre and at the core lab. Image quality was optimal in 191 and suboptimal in 222. Overall, the correlation between LVEF at the recruiting centres and at the core-lab was good, independent of image quality $\left(R^{2}=0.62\right)$. However, when a LVEF $<=30 \%$ or $>30 \%$ was used as a cut-off, about $20 \%$ of all patients would have been re-assigned to having either a LVEF above or below the cut-off, this proportion was not significantly influenced by image quality.

Conclusions: The correlation between LVEF assessed by different centres based on the same ultrasound data is good, regardless of image quality. However, one fifth of patients would have been re-assigned to a different category when using the clinically important cut-off of $30 \%$. 


\section{INTRODUCTION}

Large trials have shown a survival benefit after the implantation of an internal cardioverter defibrillator (ICD) or cardiac resynchronization therapy (CRT) in patients with a severely reduced left ventricular ejection fraction (LVEF) ${ }^{1-3}$. One of the selection criteria in these trials was the reduction of LVEF below a predefined threshold, and transthoracic echocardiography was one of the accepted imaging methods for assessing LVEF. Similarly, guidelines for the treatment of heart failure advocate the initiation of angiotensin converting enzyme inhibitor and $\beta$-blocker therapy using predefined LVEF cut-offs ${ }^{4,5}$. Therefore, important treatment decisions in this patient group with a high mortality are currently based on the measurement of LVEF by transthoracic echocardiography, both in large randomized clinical trials but also in daily clinical practice.

However, although validated as a prognostic indicator in cardiac diseases ${ }^{6,7}$ and successfully used in clinical trials to detect even small changes in ejection fraction in large patient groups ${ }^{8-10}$, the measurement of LVEF by 2 -dimensional transthoracic echocardiography is fraught with a considerable interobserver variability. 3-dimensional echocardiography and left ventricular opacification with $2^{\text {nd }}$ generation contrast media have been shown to improve the accuracy of the measurement of LVEF. However, despite recommendations by recent guidelines ${ }^{11}$, these newer methods are not yet widely used in daily clinical practice. Therefore, assessment of LVEF for the selection of therapies like ICD or CRT are most often performed by 2-dimensional echocardiography. Given the inherent risks of ICD and CRT implantation and the associated costs, the best possible accuracy in measurement of the LVEF is warranted. In this study we investigated the variability in the assessment of LVEF with 2-dimensional echocardiography, and the potential impact of this variability on treatment decisions. We also aimed to relate variability in the assessment of LVEF and its effect on treatment decisions to the image quality of the echocardiographic exams and to investigate other potential predictors of variability. We performed this analysis in a large, real world heart failure population.

\section{METHODS}

\section{Study population}

TIME-CHF was a multicenter trial comparing an intensified, BNP-guided treatment strategy with a conventional medical treatment strategy in patients aged 60 years or more with heart failure irrespective of LVEF. The design of the TIME-CHF trial has been described elsewhere in detail ${ }^{12,13}$. Briefly, patients with dyspnea (New York Heart Association class II or higher on current therapy), a history of hospitalization for heart failure within the past year, and an elevated N-terminal BNP level $(>400 \mathrm{pg} / \mathrm{ml}$ in patients $<75$ years, and $>800 \mathrm{pg} / \mathrm{ml}$ in patients $\geq 75$ years of age) were recruited in 15 tertiary and 
secondary hospital centers in Switzerland and Germany. Exclusion criteria were dyspnea not mainly due to heart failure, valvular heart disease requiring surgery, acute coronary syndrome within ten days before study inclusion, angina pectoris $>$ CCS 2 , revascularization within the month before study inclusion, body mass index $>35$, serum creatinine $>2.49 \mathrm{mg} / \mathrm{dL}$, a life expectancy of less than 3 years due to non-cardiovascular causes, inability to give informed consent, follow-up impossible, or participation in another study. All patients gave written informed consent. The study was approved by the local ethics committee of each participating center. Overall, 622 patients were included in the TIME-CHF trial.

\section{Study Protocol}

Upon inclusion in the study, patients had transthoracic echocardiography performed at the recruiting center by a board certified cardiologist trained in echocardiography. Standard clinical ultrasound equipment was used for acquisition of cine loops documenting left ventricular function from parasternal and apical acoustic windows with broadband transducers operating in harmonic imaging mode. The LVEF was determined by the treating cardiologist at the recruiting hospitals either by visual assessment or using tracking of the endothelial border and accepted mathematical models (Biplane Simpson's method or area length method). The echocardiographic studies were stored digitally and transferred to the echocardiography core laboratory at the University Hospital of Basel. Clinical data were derived from the central database of TIME-CHF. Of the 622 patients, $413(66.4 \%)$ had a complete set of echocardiographic images transmitted to the core laboratory, and comprise the patient group for the present study.

At the core laboratory, the LVEF was re-assessed by two readers (SYM and KG) blinded to results from the recruiting center. The LVEF was determined from planimetry of cineloops of the apical 4- and 2-chamber windows at end-diastole and end-systole using biplane Simpson's method according to the recommendations of the American Society of Echocardiography ${ }^{14}$, leaving the papillary muscles and trabeculations within the cavity. The interobserver variability for these two readers was determined in a randomly selected subset of 30 patients for each of the two reader by re-assessment of a third reader (BAK) blinded to the LVEF values. Interobserver variability was $4.8 \pm 3.8 \%$ for SYM and $4.5 \pm 3.3 \%$ for KG. Regional wall motion was rated for the anterior, inferior, septal lateral, and apical myocardial segments by the same readers on a 5 -point scale (1=normal, 2=mild hypokinesia, 2= severe hypokinesia, 3= akinesia, 4= dyskinesia). The presence of a 2 or more points difference in wall motion score between adjacent segments was considered to represent a regional wall motion defect. Image quality was assessed independently by a third investigator (BAK) without knowledge of the LVEF values or clinical details. Image quality was rated as bad when only 50-60\% of the endocardial border could be well visualized in any of the standard apical image planes, as fair when $60-74 \%$ of the endocardial border could be discerned, and as good when $75-100 \%$ 
of the endocardial border was visible ${ }^{15}$. For the present analysis, subjects were classified into a group with optimal image quality (those classified as having good image quality) and suboptimal image quality (those having bad or fair image quality).

\section{Statistics}

Statistical analyses were performed using SPSS Version 16.0 (SPSS Inc.). Variability was defined as the absolute difference between the two LVEF measurements. Continuous variables were compared between subgroups using a t-test or Mann-Whitney u test, as appropriate. Categorical variables were compared using Fisher's exact test. Correlations were assessed using linear regression analysis, followed by Bland Altman analysis for assessing the agreement between the two LVEFs. Multivariate linear regression was used for assessment of potential predictors of high interobserver variability. A $p$ value $<0.05$ was considered statistically significant.

\section{RESULTS}

\section{Patient characteristics}

The table shows basic parameters and clinical parameters that may be relevant to echocardiographic image quality.

Table. Clinical characteristics in the overall study patients and in patientswith suboptimal versus good image quality.

\begin{tabular}{|c|c|c|c|c|}
\hline & \multirow{3}{*}{$\begin{array}{l}\text { Overall } \\
n=413\end{array}$} & \multicolumn{2}{|c|}{ Echo quality } & \multirow{3}{*}{$\mathrm{p}$} \\
\hline & & Optimal & Suboptimal & \\
\hline & & $n=191$ & $n=222$ & \\
\hline Age (yrs $\pm S D)$ & $77.5 \pm 7.5$ & $78.0 \pm 7.3$ & $77.1 \pm 7.7$ & 0.22 \\
\hline $\mathrm{BMI}\left(\mathrm{kg} / \mathrm{m}^{2} \pm \mathrm{SD}\right)$ & $25.4 \pm 4.4$ & $24.5 \pm 4.3$ & $26.5 \pm 4.4$ & $<0.0001$ \\
\hline Male (\%) & $240(58.0)$ & $111(58.1)$ & $129(58.1)$ & 1.0 \\
\hline Systolic dysfunction $(\%)^{1}$ & $329(79.7)$ & $147(77.0)$ & $182(82)$ & 0.22 \\
\hline CAD & $281(68.0)$ & $126(66.0)$ & $145(69.8)$ & 0.46 \\
\hline RWMD & $163(39.5)$ & $77(40.3)$ & $85(38.3)$ & 0.78 \\
\hline Cardiomyopathy & $61(14.8)$ & $28(14.7)$ & 33 (19.9) & 0.87 \\
\hline COPD & $83(20.1)$ & $37(19.4)$ & $46(20.7)$ & 0.80 \\
\hline
\end{tabular}

Abbreviations: $\mathrm{BMI}=$ body mass index $\mathrm{CAD}=$ coronary artery disease, $\mathrm{RWMD}=$ regional wall motion defect, COPD $=$ chronic obstructive pulmonary disease. ${ }^{1}$ Systolic dysfunction defined as LVEF $\leq 45 \%$.

Of the 413 patients included, 191 (46\%) had a good image quality, while 157 (38\%) had a fair image quality and 65 (15.7\%) had a bad image quality. Thus, 191 (46\%) of the 
patients were classified as having an optimal image quality, and 222 (54\%) as having a suboptimal image quality. Patients in the group with suboptimal image quality had a higher body mass index. No difference was seen in the presence of systolic dysfunction, coronary artery disease, cardiomyopathy of chronic obstructive lung disease. The LVEFs assessed at the core laboratory ranged from $15 \%$ to $75 \%$, the ones assessed at the recruiting centers from $8 \%$ to $77 \% .171(41 \%)$ of the patients had a LVEF that was assessed as $\leq 35 \%$ at the core lab. Two hundred nine patients from the original TIME-CHF study population were not included in the present study, either because of the unavailability of digital image sets from two recruiting centers, or because of incomplete imaging datasets. Regarding the basic and clinical parameters, these 209 patients differed from the included patients in that they were younger $(75.8 \pm 7.6$ years vs. $77.5 \pm 7.5$ years, $\mathrm{p}=0.007)$, and in that they had a lower proportion of coronary artery disease (56.9\% vs $68.0 \%, p=0.008)$, but there were no differences regarding BMI, gender, systolic dysfunction, cardiomyopathy, COPD or LVEF as reported by the recruiting center.

\section{Variability in determination of $L V E F$}

For the whole patient population, there was a highly significant correlation between the two measurements of LVEF with an $\mathrm{R}^{2}$ of 0.62 . However, Bland-Altman analysis showed a wide $95 \%$ confidence interval of the differences ranging from -17.4 to $+17.8 \%$ despite a small overall bias of $0.2 \%$ (Figure 1), and the variability between the recruiting center and the core lab was $14.1 \pm 10.9 \%$. When the whole patient population was separated into patients with optimal image quality and patients with suboptimal image quality, the correlation for the two measurements remained highly significant for both subpopulations with an $R^{2}$ of 0.65 in the subpopulation with optimal image quality, and an $R^{2}$ of 0.59 in the subpopulation with suboptimal image quality. Bland-Altman analysis again showed small biases for both subpopulations (1.0\% in the subpopulation with good image quality, and $1.3 \%$ in the subpopulation with suboptimal image quality). However, the $95 \%$ confidence intervals were large for both subgroups, though somewhat smaller in the subpopulation with optimal image quality $195 \% \mathrm{Cl}-16.3 \%-+18.4 \%$ in the subpopulation with optimal image quality, $95 \% \mathrm{Cl}-16.5 \%-+19.1 \%$ in the subpopulation with suboptimal image quality) (Figure 2). Variability between LVEF assessment in the recruiting centers and measurements at the core lab was $14.5 \pm 10.3 \%$ for the subpopulation with optimal image quality, and $13.8 \pm 10.3 \%$ for the subgroup with suboptimal image quality ( $p=0.23$ for the difference between the 2 subgroups).

Overall, the median of the difference between the two LVEF measurements was 5.2\% (IQR 2.5-9.6). Only a limited number of significant predictors for this difference could be identified in univariate analysis. Thus, it was smaller in male subjects (median [IQR] 4.4\% [2.4-8.6\%]) compared to female (6.6 [2.6-11.1], $p=0.004)$. Furthermore, a larger difference was correlated with higher heart rate $(r=0.12, p=0.01)$, shorter $Q R S$ duration ( $r=-0.11, p=0.03$ ), and smaller LV ventricles (end-diastolic volume of LV 
[LVEDV] $r=-0.17, p=0.001)$. Other predictors were not significantly correlated with difference between the two measurements, particularly body mass index, presence of COPD, cause of heart failure, and the centre where initial assessment was done. In multivariate analysis, the only independent predictor was the LVEDV.

163 patients (39\%) in the whole study population had regional wall motion abnormalities. These patients showed a highly significant correlation between the two measurements of LVEF ( $R^{2}$ of $\left.0.38, p<0.0001\right)$. Again, Bland-Altman analysis showed a wide $95 \%$ confidence interval of the differences ranging from -15.5 to $+17.1 \%$ with an overall bias of $0.8 \%$.
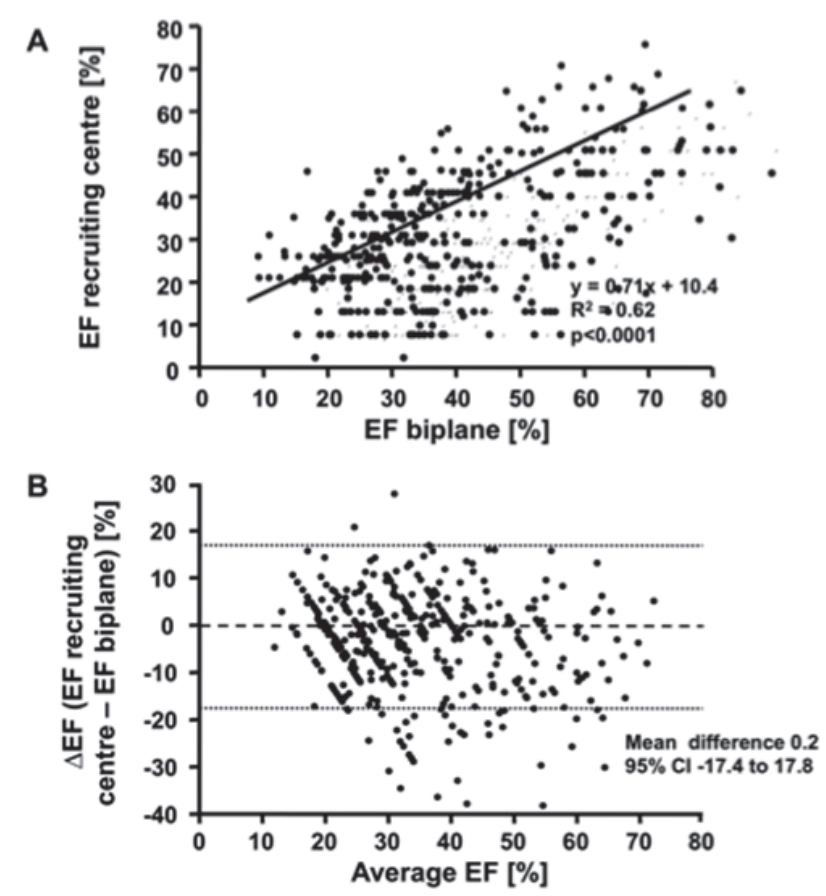

Fig. 1. (A) Correlation between LVEF measured at the recruiting center and biplane LVEF measured at the core laboratory by linear regression analysis (solid line) for the whole patient group. (B) Bland-Altmann plot of the same data, dashed lines specify mean difference of the measurements, dotted lines the corresponding $95 \%$ confidence interval (Cl). 
A

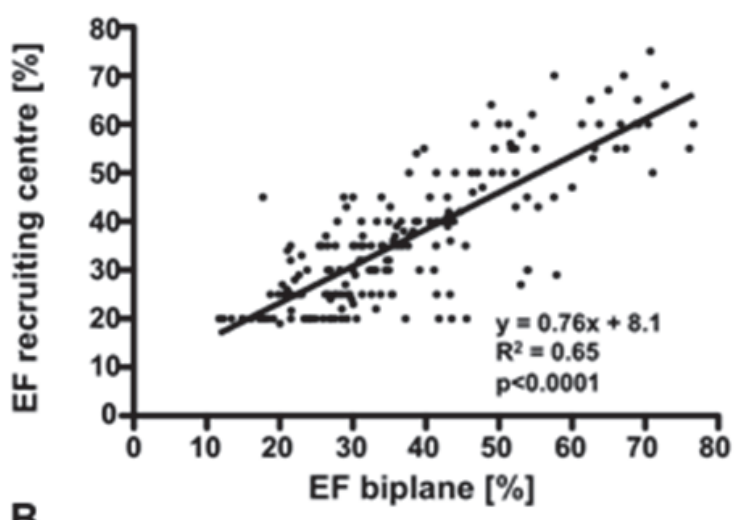

B

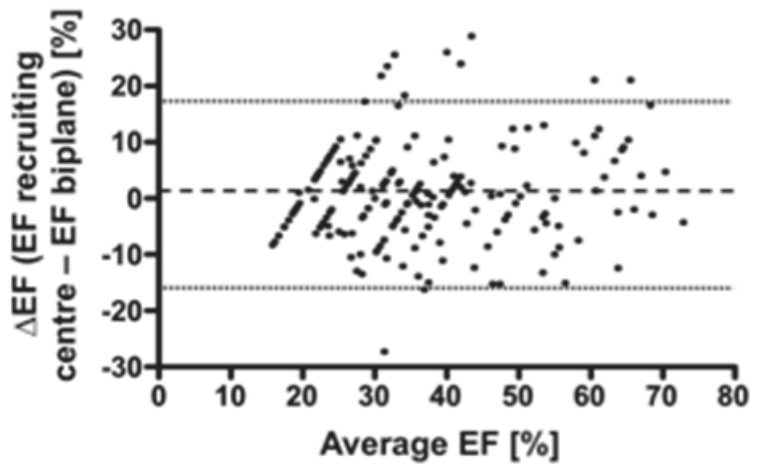

C
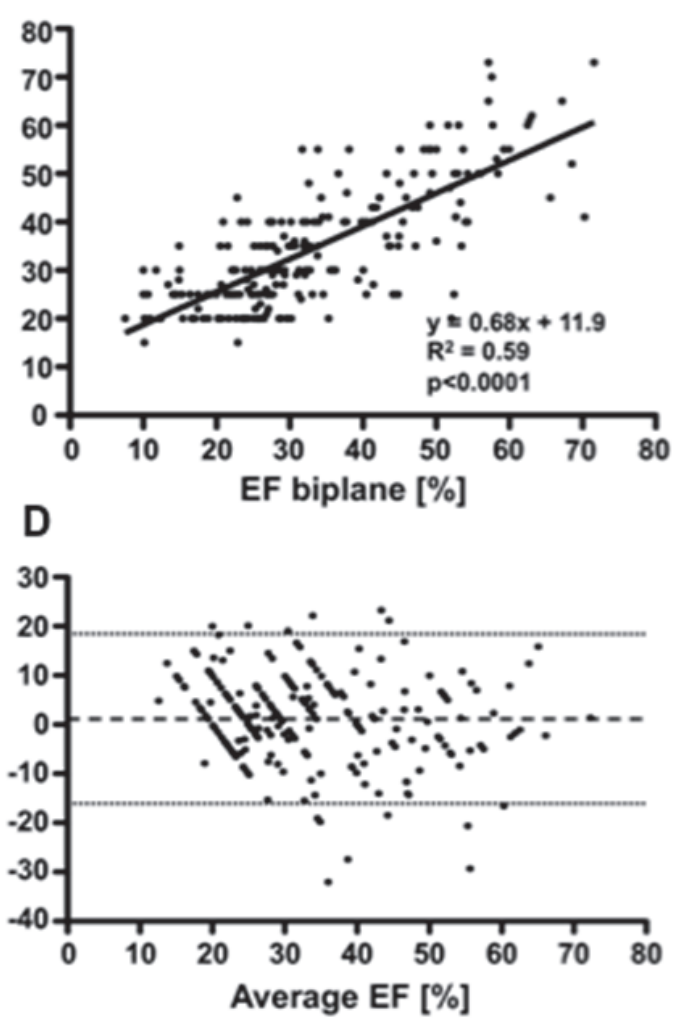

Fig. 2. (A) Correlation between LVEF measured th the recruiting center and biplane LVEF measured at the core laboratory by linear regression anatysis (solid line) for the patients with optimal image quality. (B) BlandAltmann plot of the same data, dashed lines specify mean difference of the measurements, dotted lines the corresponding 95\% confidence interval (Cl). (C) Correlation between LVEF measured the recruiting center and biplane LVEF measured al the core laboratory for the patients with suboptimal image quality. (D) BlandAltmamm plot of the same data.

Potential influence of the reliability in determination of LVEF on clinical decision-making Discrete LVEF thresholds are used for important clinical decisions, and therefore we examined what potential influence the intercenter reliability in the determination of LVEF could have on situating an individual patient above or below commonly used threshold values (Figure 3). For a threshold of an LVEF of $30 \%$, in the whole patient population, $21.1 \%$ of all patients changed from either $\leq 30 \%$ to $>30 \%$ or vice versa. For the same threshold, the percentages of re-assignment were $23.0 \%$ for patients with optimal image quality and $19.4 \%$ for patients with suboptimal image quality ( $p$ for the difference in proportions 0.40). For a threshold of an LVEF of $\leq 35 \%, 16.9 \%$ were reassigned in the whole patient population. For patients with optimal image quality the percentage of re-assignment was $16.2 \%$, for patients with suboptimal image quality $17.6 \%$ ( $p$ for the difference in proportions 0.79 ). For a threshold of an LVEF of $\leq 40 \%$, $13.6 \%$ were re-assigned in the whole patient population. For patients with optimal image quality the percentage of re-assignment was $13.1 \%$, for patients with suboptimal 
image quality $14.1 \%$ ( $p$ for the difference in proportions 0.39 ). For the threshold of a normal LVEF of $\geq 55 \%, 6.3 \%$ were re-assigned in the whole patient population, $5.9 \%$ in the subpopulation with optimal image quality, and $6.8 \%$ in the subpopulation with suboptimal image quality ( $p$ for the difference in proportions 0.69 ).

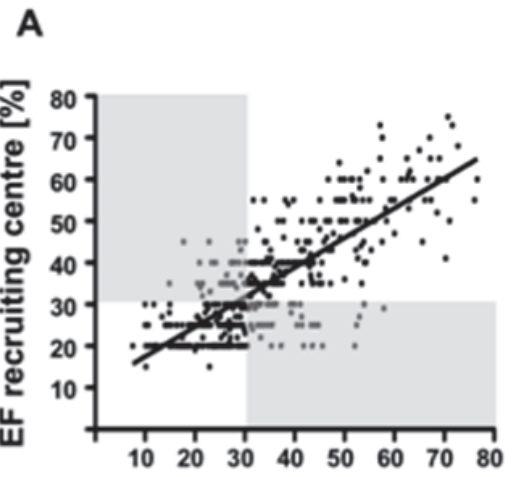

B

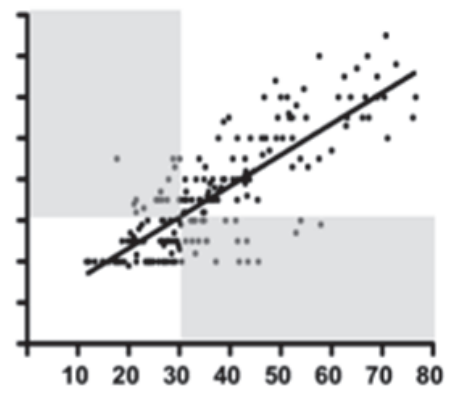

EF biplane [\%]
C

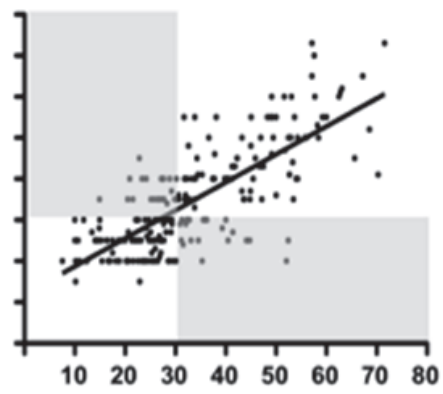

$\begin{array}{llllllll}10 & 20 & 30 & 40 & 50 & 60 & 70 & 80\end{array}$

Fig. 3. Correlation of LVEF measured at the recruiting center and biplane LVEF measured at the core laboratory for (A) the whole patient group, (B) patients with optimal image quality, and (C) patients with suboptimal image quality. Those datapoints for which a reclassification from $\leq 30 \%$ to $>30 \%$ occurred are shaded in grey.

Fig. 3. Correlation of LVEF measured at the recruiting center and biplane LVEF measured at the core laboratory for (A) the whole patient group, (B) patients with optimal image quality, and (C) patients with suboptimal image quality. Those datapoints for which a reclassification from $\leq 30 \%$ to $>30 \%$ occurred are shaded in grey.

\section{DISCUSSION}

Our study shows a high variability in the evaluation of LVEF in a large study of patients with heart failure with both reduced ejection fraction and normal ejection fraction. This high variability resulted despite the fact that the same image material was used for analysis at the recruiting center and at the echocardiography core laboratory. Using commonly accepted cut-offs for the implantation of ICD or CRT, 15-20\% of all patients were re-classified as having a LVEF above or below the cut-offs when the images were re-assessed. Neither the variability in LVEF assessment between hospital centers nor the percentages of patients re-classified depended significantly on the quality of the acquired ultrasound images. The only independent predictor of a high variability in LVEF measurements was a smaller left ventricle.

Interobserver reliability. The numeric assessment of LVEF is the single most important measurement in cardiology with a profound influence on diagnosis and management of patients. Hence, it is of utmost importance that this measurement is reliable and reproducible. While other imaging modalities in use in current cardiology practice (angiography, SPECT, MRI, CT, RNA) can provide measurements of LVEF, two- 
dimensional echocardiography is by far the most commonly employed method. Twodimensional echocardiography for the measurement of LVEF relies on either a visual assessment of ventricular function, or on tracings of the endocardial borders and calculation of LVEF using geometric models (Simpson's biplane analysis, area length method). In comparison to other imaging modalities, two-dimensional echocardiography has the disadvantages of (I) dependence on unequivocal endocardial border delineation, which is not uniformly achieved in all echocardiographic images, and (II) reliance on geometric assumptions in the case of LVEF measurement by Simpson's biplane method or area length method. The accuracy of these echocardiographic methods for the assessment of LVEF in comparison with SPECT, MRI and CT has been studied extensively ${ }^{16-18}$ and overall correlations have been shown to be good. Thus, 2-dimensional echocardiography is a valuable tool for assessing treatment effects in therapeutic trials. However, there has been concern regarding interstudy, interobserver and intraobserver variability of 2-dimensional echocardiographic measurements of LVEF, especially with regards to the serial assessment of changes in ejection fraction, and inclusion or exclusion of subjects into studies where LVEF thresholds are used as inclusion criteria. By the design of the present study, the LVEF assessment at the recruiting hospital was done either by visual assessment, Simpson's biplane or area length assessment, and thus the 95\% confidence interval of the differences do not represent a strict measurement of interobserver variability. Nevertheless, the variabilities in our study correspond to previously published values of interobserver variability ${ }^{19,20}$, while others have published lower values ${ }^{21-23}$. An important difference between the cited studies and our data is that we investigated the variability between hospital centers in the assessment of LVEF in a large, real world clinical heart failure trial, while the cited studies were conducted specifically to assess the accuracy and interobserver variability of different imaging methods for the assessment of LVEF in a lower number of subjects. Thus, our study can be assumed to more reliably indicate the variability of LVEF measurements as performed in routine cardiology practice. The variability in the assessment of LVEF in our study was largely independent of the quality of the echocardiographic images. Others have described a better agreement for echocardiography with other imaging techniques in those patients with better image quality ${ }^{15}$. In our study, $53.8 \%$ of patients were deemed to have a suboptimal image quality, which seems to be a relatively high number. The large percentage of patients with suboptimal image quality may be due to the fact that a real world population was examined. More importantly, the assessment of image quality was performed at the core lab to assess its effect on the reliability in measurement of LVEF and reclassification above or below a threshold. To our own surprise, image quality did not have a significant impact on variability, and thus image quality did not impact the main study results. However, our dataset is likely to more closely reflect a real-world situation than data from dedicated imaging studies. Importantly, only a very limited number of factors potentially influencing the variability could be 
indentified in this study. Of these variables, only the enddiastolic volume of the LV was an independent, albeit very limited predictor.

Reclassification. A large proportion of patients were reclassified below or above clinically relevant thresholds when the echocardiographic images were reassessed. These proportions were similar for cut-offs of $30 \%, 35 \%$, and $40 \%$, all of which are important for clinical decision-making. The proportion of reclassification was lower regarding the cut-off of a normal LVEF, but this seems to be due to the smaller number of patients included in this study with normal LVEF. Previous information on reclassification is very limited. Thus, Chuang et al compared 2 dimensional echocardiography with CMR with regards to classification of LVEF as normal ( $255 \%$ ), depressed (LVEF $>35 \%$ to $55 \%$ ) or severely depressed $(\leq 35 \%)$ in a total of 35 patients, and found that up to $44 \%$ of patients were classified differently by echocardiography. Similarly, Ray et al. ${ }^{24} \mathrm{com}-$ pared 2D echocardiography to radionuclide ventriculography in 70 patients, and found that $40 \%$ of patients would have been classified differently depending on the imaging test used. Given the far-reaching consequences of placing individuals above or below a certain LVEF threshold, especially with regards to device implantation, the high rate of reclassification in our study is worrying, as it may lead to higher numbers needed to treat both in clinical trials, but also in real world patients. Contrast-enhanced echocardiography and three-dimensional echocardiography have been shown to improve both the accuracy of LVEF determinations when compared to reference methods, as well as to reduce interobserver variability ${ }^{20,25}$. However, the benefit of these new techniques with regards to misclassification of patients above or below LVEF cutoffs remains to be determined. In addition, our data indicate that studies, especially single center studies, that are specifically designed to test the accuracy and reproducibility of imaging methods, tend to underestimate the measurement variability observed in clinical practice.

Study limitations. LVEF assessment at the recruiting hospital centers was done according to the preferences of the investigators with either visual assessment or biplane Simpson's method, whereas in the echocardiographic core laboratory all LVEFs were measured using biplane Simpson's method. Thus, we do not report a true interobserver variability. This may have increased the variability we report in this study. Also, the fact that the readers at the core lab, but not the cardiologist at the recruiting center were blinded for the clinical characteristics of the patient might have further increased variability. However, differences in the methodology used for LVEF assessment are the reality in clinical practice, and thus our data closely reflect the true variability of LVEF measurements with two-dimensional echocardiography in daily clinical practice. Also, the assessment of echocardiographic image quality was done on a subjective basis, while others have assessed image quality based on criteria that take into account the extent of endocardial border visualization ${ }^{21,25}$. However, there are no universally accepted criteria for image quality. For the purpose of assessing the influence of image quality on LVEF, which is a global measurement of left ventricular function, in our view, a subjective assessment is sufficient, particularly because this is also done accordingly in daily 


\section{CHAPTER 3}

clinical practice. Furthermore, the influence of image quality on the variability was very limited in this study.

Conclusions. Conventional 2D echocardiographic assessment of LVEF carries with it a considerable variability. This variability is not dependent on overall image quality and identification of factors potentially influencing this variability is very limited. A significant proportion of patients (i.e. 15-20\%) would have been re-assigned to a different LVEF category upon reassessment of the echocardiographic images. Thus, the reported variability in LVEF assessment appears to have an important potential impact on clinical decision-making, especially on the indication for the implantation of an ICD or CRT device. Whether the standard use of biplane Simpson's method for LVEF calculations or other imaging modalities would reduce variability of LVEF assessment and its impact on clinical decision-making, and, importantly, would also be applicable in clinical practice remains to be determined.

\section{Conflict of interest}

none declared

\section{Sources of funding}

Dr. Kaufmann is supported by a SCORE grant (SNF 32323B_123919/1) from the Swiss National Science Foundation. TIME-CHF was supported by the Horten Research Foundation (Lugano, Switzerland; $>55 \%$ of the study's budget), as well as by smaller unrestricted grants from AstraZeneca Pharma, Novartis Pharma, Menarini Pharma, Pfizer Pharma, Servier, Roche Diagnostics, Roche Pharma, and Merck Pharma. 


\section{REFERENCES}

1. Moss AJ, Zareba W, Hall WJ, Klein H, Wilber DJ, Cannom DS, Daubert JP, Higgins SL, Brown MW, Andrews ML. Prophylactic implantation of a defibrillator in patients with myocardial infarction and reduced ejection fraction. N Engl J Med. 2002;346:877-883

2. Bardy GH, Lee KL, Mark DB, Poole JE, Packer DL, Boineau R, Domanski M, Troutman C, Anderson J, Johnson G, McNulty SE, Clapp-Channing N, Davidson-Ray LD, Fraulo ES, Fishbein DP, Luceri RM, Ip JH. Amiodarone or an implantable cardioverter-defibrillator for congestive heart failure. N Engl J Med. 2005;352:225-237

3. Bristow MR, Saxon LA, Boehmer J, Krueger S, Kass DA, De Marco T, Carson P, DiCarlo L, DeMets D, White BG, DeVries DW, Feldman AM. Cardiac-resynchronization therapy with or without an implantable defibrillator in advanced chronic heart failure. N Eng/ J Med. 2004;350:2140-2150

4. Hunt SA, Abraham WT, Chin MH, Feldman AM, Francis GS, Ganiats TG, Jessup M, Konstam MA, Mancini DM, Michl K, Oates JA, Rahko PS, Silver MA, Stevenson LW, Yancy CW. 2009 focused update incorporated into the acc/aha 2005 guidelines for the diagnosis and management of heart failure in adults: A report of the american college of cardiology foundation/american heart association task force on practice guidelines: Developed in collaboration with the international society for heart and lung transplantation. Circulation. 2009;119:e391-479

5. Dickstein K, Cohen-Solal A, Filippatos G, McMurray JJ, Ponikowski P, Poole-Wilson PA, Stromberg A, van Veldhuisen DJ, Atar D, Hoes AW, Keren A, Mebazaa A, Nieminen M, Priori SG, Swedberg K. Esc guidelines for the diagnosis and treatment of acute and chronic heart failure 2008: The task force for the diagnosis and treatment of acute and chronic heart failure 2008 of the european society of cardiology. Developed in collaboration with the heart failure association of the esc (hfa) and endorsed by the european society of intensive care medicine (esicm). Eur Heart J. 2008;29:2388-2442

6. White HD, Norris RM, Brown MA, Brandt PW, Whitlock RM, Wild CJ. Left ventricular end-systolic volume as the major determinant of survival after recovery from myocardial infarction. Circulation. 1987;76:44-51

7. Wong M, Johnson G, Shabetai R, Hughes V, Bhat G, Lopez B, Cohn JN. Echocardiographic variables as prognostic indicators and therapeutic monitors in chronic congestive heart failure. Veterans affairs cooperative studies v-heft i and ii. V-heft va cooperative studies group. Circulation. 1993;87:VI65-70

8. Randomised, placebo-controlled trial of carvedilol in patients with congestive heart failure due to ischaemic heart disease. Australia/new zealand heart failure research collaborative group. Lancet. 1997;349:375-380

9. Effect of enalapril on survival in patients with reduced left ventricular ejection fractions and congestive heart failure. The solvd investigators. N Engl J Med. 1991;325:293-302

10. Effects of metoprolol $\mathrm{cr}$ in patients with ischemic and dilated cardiomyopathy : The randomized evaluation of strategies for left ventricular dysfunction pilot study. Circulation. 2000;101:378-384

11. Lang RM, Badano LP, Tsang W, Adams DH, Agricola E, Buck T, Faletra FF, Franke A, Hung J, de Isla LP, Kamp O, Kasprzak JD, Lancellotti P, Marwick TH, McCulloch ML, Monaghan MJ, Nihoyannopoulos P, Pandian NG, Pellikka PA, Pepi M, Roberson DA, Shernan SK, Shirali GS, Sugeng L, Ten Cate FJ, Vannan MA, Zamorano JL, Zoghbi WA. Eae/ase recommendations for image acquisition and display using threedimensional echocardiography. J Am Soc Echocardiogr. 2012;25:3-46

12. Pfisterer $M$, Buser $P$, Rickli $H$, Gutmann $M$, Erne $P$, Rickenbacher $P$, Vuillomenet $A$, Jeker $U$, Dubach $P$, Beer H, Yoon SI, Suter T, Osterhues HH, Schieber MM, Hilti P, Schindler R, Brunner-La Rocca HP. Bnp-guided vs symptom-guided heart failure therapy: The trial of intensified vs standard medical therapy in elderly patients with congestive heart failure (time-chf) randomized trial. JAMA. 2009;301:383-392

13. Brunner-La Rocca HP, Buser PT, Schindler R, Bernheim A, Rickenbacher P, Pfisterer M. Management of elderly patients with congestive heart failure--design of the trial of intensified versus standard medical therapy in elderly patients with congestive heart failure (time-chf). Am Heart J. 2006;151:949-955

14. Lang RM, Bierig M, Devereux RB, Flachskampf FA, Foster E, Pellikka PA, Picard MH, Roman MJ, Seward J, Shanewise JS, Solomon SD, Spencer KT, Sutton MS, Stewart WJ. Recommendations for chamber quantification: A report from the american society of echocardiography's guidelines and standards committee 


\section{CHAPTER 3}

and the chamber quantification writing group, developed in conjunction with the european association of echocardiography, a branch of the european society of cardiology. J Am Soc Echocardiogr. 2005;18:14401463

15. Tighe DA, Rosetti M, Vinch CS, Chandok D, Muldoon D, Wiggin B, Dahlberg ST, Aurigemma GP. Influence of image quality on the accuracy of real time three-dimensional echocardiography to measure left ventricular volumes in unselected patients: A comparison with gated-spect imaging. Echocardiography. 2007;24:1073-1080

16. Erbel R, Schweizer P, Lambertz H, Henn G, Meyer J, Krebs W, Effert S. Echoventriculography -- a simultaneous analysis of two-dimensional echocardiography and cineventriculography. Circulation. 1983;67:205215

17. Schalla S, Nagel E, Lehmkuhl H, Klein C, Bornstedt A, Schnackenburg B, Schneider U, Fleck E. Comparison of magnetic resonance real-time imaging of left ventricular function with conventional magnetic resonance imaging and echocardiography. Am J Cardiol. 2001;87:95-99

18. Bellenger NG, Burgess MI, Ray SG, Lahiri A, Coats AJ, Cleland JG, Pennell DJ. Comparison of left ventricular ejection fraction and volumes in heart failure by echocardiography, radionuclide ventriculography and cardiovascular magnetic resonance; are they interchangeable? Eur Heart J. 2000;21:1387-1396

19. Chuang ML, Hibberd MG, Salton CJ, Beaudin RA, Riley MF, Parker RA, Douglas PS, Manning WJ. Importance of imaging method over imaging modality in noninvasive determination of left ventricular volumes and ejection fraction: Assessment by two- and three-dimensional echocardiography and magnetic resonance imaging. J Am Coll Cardiol. 2000;35:477-484

20. Hoffmann R, von Bardeleben S, ten Cate F, Borges AC, Kasprzak J, Firschke C, Lafitte S, Al-Saadi N, KuntzHehner S, Engelhardt M, Becher H, Vanoverschelde JL. Assessment of systolic left ventricular function: A multi-centre comparison of cineventriculography, cardiac magnetic resonance imaging, unenhanced and contrast-enhanced echocardiography. Eur Heart J. 2005;26:607-616

21. Whalley GA, Gamble GD, Walsh HJ, Wright SP, Agewall S, Sharpe N, Doughty RN. Effect of tissue harmonic imaging and contrast upon between observer and test-retest reproducibility of left ventricular ejection fraction measurement in patients with heart failure. Eur J Heart Fail. 2004;6:85-93

22. Shahgaldi K, Gudmundsson P, Manouras A, Brodin LA, Winter R. Visually estimated ejection fraction by two dimensional and triplane echocardiography is closely correlated with quantitative ejection fraction by real-time three dimensional echocardiography. Cardiovasc Ultrasound. 2009;7:41

23. Cannesson M, Tanabe M, Suffoletto MS, McNamara DM, Madan S, Lacomis JM, Gorcsan J, 3rd. A novel two-dimensional echocardiographic image analysis system using artificial intelligence-learned pattern recognition for rapid automated ejection fraction. J Am Coll Cardiol. 2007;49:217-226

24. Ray SG, Metcalfe MJ, Oldroyd KG, Pye M, Martin W, Christie J, Dargie HJ, Cobbe SM. Do radionuclide and echocardiographic techniques give a universal cut off value for left ventricular ejection fraction that can be used to select patients for treatment with ace inhibitors after myocardial infarction? $\mathrm{Br}$ Heart J. 1995;73:466-469

25. Jenkins C, Bricknell K, Hanekom L, Marwick TH. Reproducibility and accuracy of echocardiographic measurements of left ventricular parameters using real-time three-dimensional echocardiography. J Am Coll Cardiol. 2004;44:878-886 


\section{CHAPTER 4}

Head-to-Head Comparison of Two- and Three-Dimensional Echocardiographic Methods for Left Atrial Chamber Quantification with Magnetic Resonance Imaging

Ronny R. Buechel, MD, Gregor Sommer, MD, Frank Peter Stephan, MD, Jens Bremerich, MD, Michael J. Zellweger, MD and Beat A. Kaufmann, MD

J Am Soc Echocardiogr. 2013; 26: 428-435 


\section{CHAPTER 4}

\section{ABSTRACT}

Background: Limited data is available on the accuracy of quantification methods for left atrial (LA) volumes using two-dimensional (2DE) and particularly real-time threedimensional (RT3DE) echocardiographic methods in comparison with a reference standard. Our aim was to perform a head-to-head comparison between 2DE and RT3DE methods with magnetic resonance imaging (MRI) as standard of reference.

Methods: LA volumes derived from 2DE methods (i.e. biplane modified Simpson's, biplane area-length and prolate ellipse method) and from RT3DE methods (i.e. 4D LA Analysis and $Q L A B$ ) of sixty consecutive patients were compared to MRI. Offline analysis time was recorded.

Results: Biplane modified Simpson's and the area-length method showed good intraclass correlation with MRI for maximum ( $r=0.70$ and $r=0.69, p<0.001)$ and minimum $(r=0.83$ and $r=0.82, p<0.001)$ volumes. While RT3DE methods led to a moderate increase in correlation for maximum $(r=0.94$ and $0.70, p<0.001)$ and minimum $(r=0.95$ and $r=0.90, p<0.001$ ) volumes and narrower Bland-Altman limits of agreement than 2DE methods, offline analysis time was higher for RT3DE (155-161 versus 103-144 seconds).

Conclusions: Simpson's and area-length methods offer reasonable accuracy for LA chamber quantification across a broad range of volumes, while RT3DE methods lead to a moderate improvement in accuracy at the cost of more elaborate offline analysis. 


\section{INTRODUCTION}

As a consequence of the ongoing technical advancements of echocardiography, novel methods for the measurement of left atrial (LA) volumes have been developed. While M-Mode measurement of the LA anterior-posterior diameter represents a simple unidimensional assessment of LA size, the introduction of two-dimensional echocardiography $(2 D E)$ has led to volume based methods. The latter, namely the biplane arealength formula or the biplane modified Simpson's rule, are recommended by the guidelines for measuring LA volumes because of their higher accuracy and stronger prognostic value. ${ }^{1,2}$ 2DE methods, however, rely heavily on estimates based mathematical formulas based on geometrical assumptions. Finally, the advent of real-time threedimensional echocardiography (RT3DE) has enabled volumetric and functional quantification based on real anatomical configurations. Initially, RT3DE LA analysis was performed using free hand ${ }^{3}$ or semi-automated slice-by-slice contouring, ${ }^{4,5}$ and later by the utilization of software analysis tools using semi-automated contour-tracing or edgedetection algorithms originally developed for left ventricular quantification. ${ }^{6,7}$ Software tools specifically dedicated to LA quantification have only recently been introduced. ${ }^{8}$

The accuracy of LA assessment may be of clinical importance as it has repeatedly been suggested that size and function serve as an independent predictor for adverse outcomes in a variety of clinical conditions such as myocardial infarction, atrial fibrillation, or heart failure. ${ }^{9-15}$ Even though some of the available techniques for LA chamber quantification have been compared with each other ${ }^{4,5,16-18}$ and to some extent have been validated against independent reference standards such as magnetic resonance imaging (MRI) or computer tomography, ${ }^{6-8,19-21}$ there remains a lack of comprehensive data comparing 2DE quantification methods and the more recently established RT3DE techniques against an independent reference standard. Particularly, the gain in accuracy through the use of presumably more elaborate RT3DE techniques remains to be elucidated.

Therefore, the aim of the present study was to perform a comprehensive head-tohead comparison of commonly available techniques for LA chamber quantification using 2DE and 3DRTE with cardiac MRI serving as standard of reference. ${ }^{20}$ 


\section{METHODS}

\section{Patient Population}

Sixty consecutive patients scheduled for pulmonary vein isolation due to symptomatic persistent or paroxysmal atrial fibrillation who underwent clinically indicated cardiac MRI were prospectively enrolled in the present study. Echocardiography was performed in all patients on the same day as MRI and patients were enrolled regardless of the quality of the acoustic window obtained during acquisition. Informed consent was obtained from all patients and the study protocol was approved by the local institutional review board.

\section{Echocardiographic Image Acquisition and Quantification}

Image acquisition was performed in all participants in the lateral recumbent position using a commercially available echocardiography system (iE33, Philips Medical Systems) equipped with a 2.5-MHz to 3.5-MHz matrix array transducer (X3-1 and X5-1, Philips Medical Systems) by a trained sonographer and following a standardized protocol. Parasternal long-axis and apical long-axes were acquired for 2D imaging. For RT3DE, an apical view enabling full coverage of the LA was selected and lateral sector size was carefully adjusted to achieve the highest possible frame rate during image acquisition. Trigger delay was set to 300ms after the electrocardiographic QRS complex to ensure temporal coverage of the entire diastole using a full-volume loop. The gain settings were adjusted to a high-midrange level to allow for additional adjustments during postprocessing. All datasets were acquired during a breathhold. Two to four datasets were obtained per patient. All datasets were transferred to a dedicated workstation for offline analysis. Measurements of 3D LA volumes were performed with two different 3D quantification software packages: QLAB Advanced Quantification (Version 8.1, Philips Medical Systems) and 4D LA Analysis (TomTec Imaging Systems). QLAB was initially developed for left ventricular analysis and requires identification of five anatomic landmarks (septal, lateral, anterior, and inferior mitral annulus and posterior wall of the left atrium) at end-diastole and end-systole. Automatic edge detection is then performed, and LA borders are tracked throughout the entire cardiac cycle (Figure 1). In contrast, 4D LA Analysis (TomTec Imaging Systems) is a novel software analysis tool developed specifically for RT3DE analysis of the LA. Using this analysis tool, the reader first identifies mitral valve closure and mitral valve opening to manually define the end-diastolic frame representing minimum LA volume and the end-systolic frame representing maximum LA volume. In a further step, initial contours of the LA at end-diastole and endsystole are manually defined for the apical four-chamber, two-chamber, and long-axis view (Figure 2). A polyhedron model of the LA is then automatically created by the software tool using an automated border-detection technique. In the following step the 
contours are manually corrected, if necessary. Pulmonary vein orifices and/or left atrial appendage were not included into the contour. For both methods, maximum ( $\left(\mathrm{A}_{\max }\right)$ and minimum $\left(L A_{\min }\right) L A$ volumes were calculated.
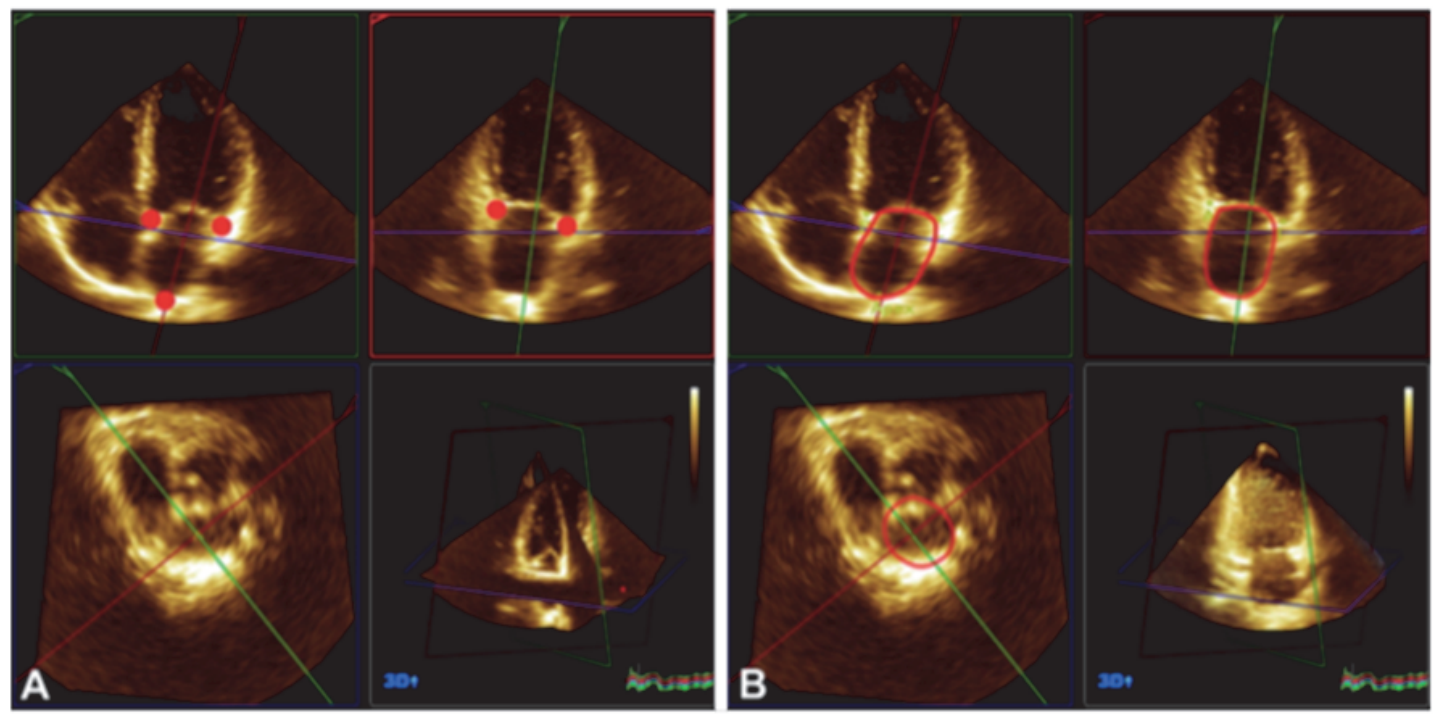

Fig. 1. Left atrial chamber quantification using QLAB. Semi-automatic left atrial border tracing by placing markers at 4 mitral annular points (lateral, septal. inferior, anterior) and an atrial superior dome point opposite the annulus (A). The automatic border tracing is then shown by the software (B). before the corresponding volumes are calculated in a further step. (Point markers and contours are manually enhanced for visibility purposes).
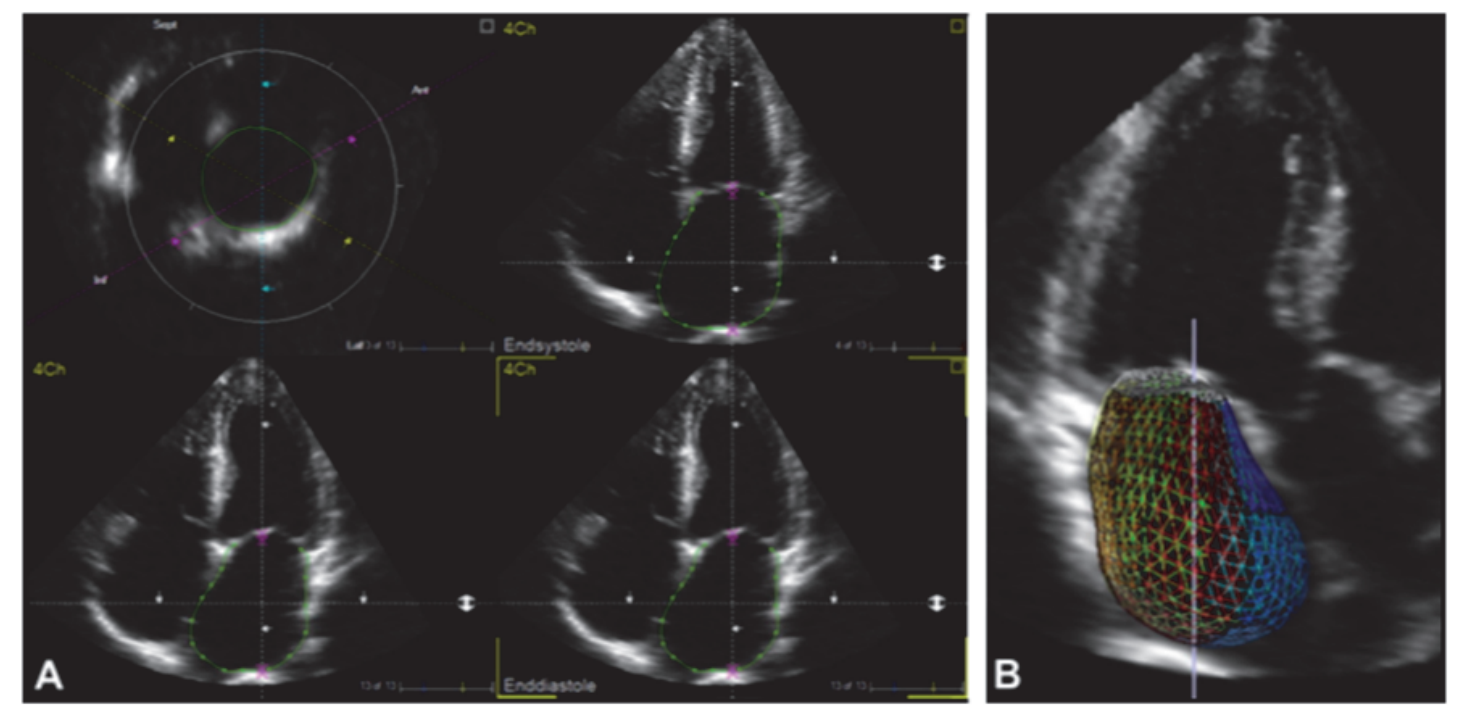

Fig. 2. Left atrial chamber quantification using 40 LA Analysis. Manual contouring is required at end-systole and end-diastole in apical 4-chamber (A), 3-chamber, and 2-chamber views. before a polyhedron-model (B) is automatically created based on left atrial endocardial border tracing and the corresponding volumes are calculated. 
For 2DE LA volume quantification, three commonly used methods were applied: The biplane area-length method $(A L)$ uses the formula $V=8(A 1)(A 2) / 3 \pi(L)$, where $A 1$ and A2 represent areas obtained from LA planimetry in a four-chamber and two-chamber view, and $L$ is the shortest length from center of the mitral annular plane to the superior aspect of the LA (Figure 3, Panel A and B). The biplane modified Simpson's rule (Simpson), assuming the stacked disks are circular, uses the formula $V=\pi / 4(L) \sum(A 1)(A 2)$, where $V$ is volume, $L$ is the length from the center of the mitral annular plane to the superior aspect of the $L A$, and $A 1$ and $A 2$ represents the 20 discs obtained from fourchamber and two-chamber views (Figure 3, Panel A and B). The prolate ellipse method $(P E)$, using the formula $V=0.523(D 1)(D 2)(D 3)$, where $D 1$ is measured from the middle of the plane of the mitral annulus to the superior aspect of the LA in a four-chamber view, D2 is the orthogonal short-dimension to D1, and D3 reflects the anterior-posterior diameter (AP) measured in a parasternal long-axis (Figure 3, Panel $C$ and D). All 2DE measurements were performed at end-diastole and end-systole to obtain $L A_{\max }$ and $L A_{\text {min. }}$. As with RT3DE, end-diastole and end-systole was identified using mitral valve closure and mitral valve opening as a reference point to assure identical timings.
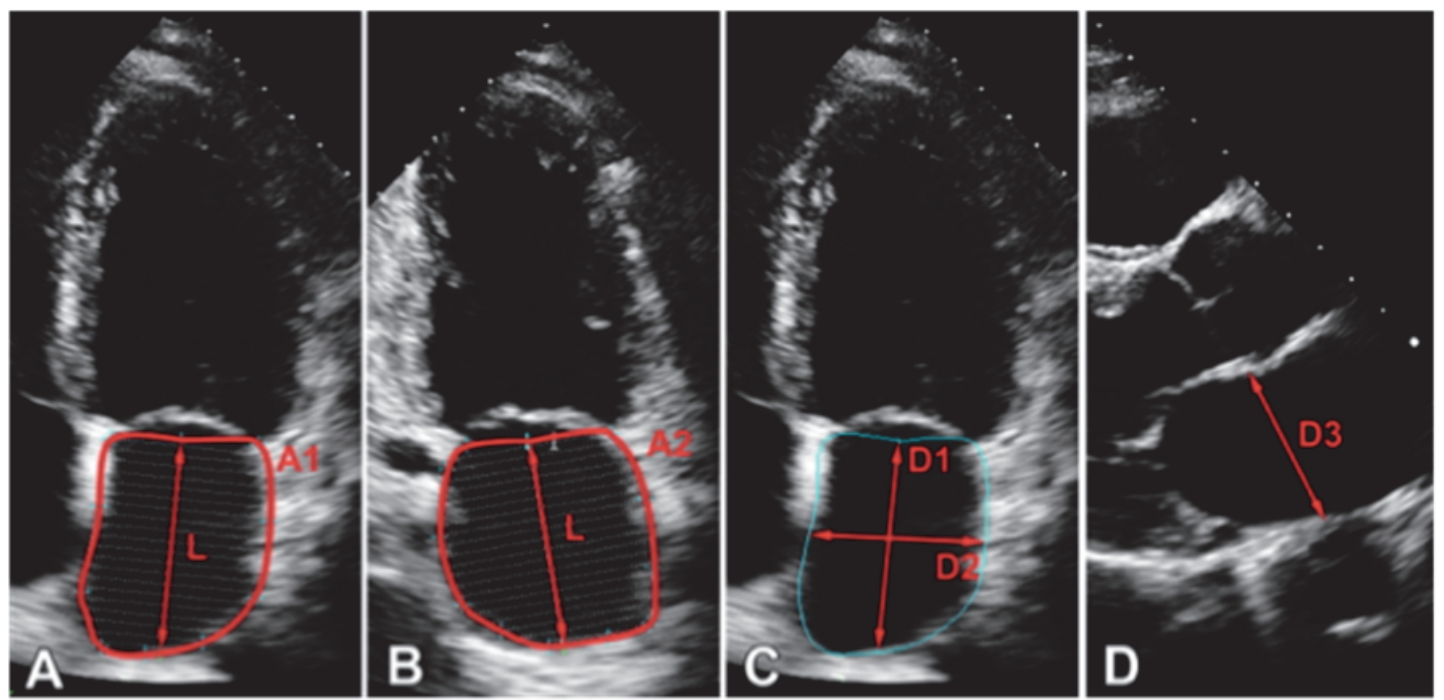

Figure 3. Left atrial chamber quantification using the area-length method and biplane modified Simpson's rule (A and B). A1 and A2. represent areas acquired in apical long-axis views consisting of a stack of 20 disks for Simpson's rule, while $\mathrm{L}$ is the atrial length measured from the middle of the mitral annular plane to the back wall. For the prolate ellipse method ( $C$ and $D)$, D1 is measured from the middle of the plane of the mitral annulus to the superior aspect of the LA in a four-chamber view, while D2 is the orthogonal short-dimension to D1. D3 represents the anterior-posterior diameter measured in a parasternal long-axis.

Analysis time in seconds was recorded for all echocardiographic methods from the time the datasets were loaded into the respective software tool until $L A_{\max }$ and $L A_{\min }$ were calculated.

Intraobserver and interobserver agreement was assessed by repeated measurement from 15 randomly selected subjects at least two months after the first analysis. The 
second observer used the same datasets for offline analysis as the first observer but was blinded to the results or the identity of the subjects. A dedicated workstation was used for analysis by the second observer.

\section{MR Image Acquisition and Quantification}

A 1.5 T clinical system (Magnetom Espree, Siemens Medical Solutions, Erlangen Germany) with multi-channel phased-array receiver coils (Total imaging matrix, Siemens Medical Solutions) was used to perform MRI acquisition with the patients in supine position. Localizing scans were followed by a series of transversely oriented cine acquisitions using a balanced steady state free precession ( $b$-SSFP) sequence (TR=40ms, TE $=1.2 \mathrm{ms,}$ flip angle $63^{\circ}$, matrix $192 \times 156$, in plane resolution $1.6 \times 1.6 \mathrm{~mm}^{2}$ ). Fifteen to twenty slices were acquired during breath-hold at inspiration to cover the the entire LA and the left ventricular mitral anular plane with retrospective electrocardiogram gating. Section thickness was $6 \mathrm{~mm}$ without inter-slice gaps. Temporal resolution was 25 frames per heart cycle using parallel imaging with an acceleration factor of 2.

MRI datasets were transferred to a dedicated workstation and were analyzed by an experienced radiologist blinded to the results of echocardiography. A dedicated software tool was used (Argus, Siemens Medical Solutions) and the LA border was contoured in axial slices. Pulmonary vein orifices and/or left atrial appendage were not included into the contour. Timing of LA volume measurements and calculation of functional parameters were identical to that described for echocardiographic acquisition methods. Namely, mitral valve closure and opening was used as a reference point to identify end-diastole and end-systole.

\section{Statistics}

SPSS 18 (SPSS Inc.) was used for statistical testing. Quantitative variables were expressed as mean \pm 1 standard deviation and categorical variables as frequencies or percentages. Paired t-test and Wilcoxon rank-sum test were used for parametric and non-parametric variables, respectively, to test for differences between parameters acquired during the scans. The correlation of dimensional and functional parameters between echocardiographic volumes and MRI was assessed using intraclass correlation analysis (absolute agreement). A test using Fisher's z transformation was applied to test for differences between correlation coefficients. ${ }^{22}$ In addition, Bland-Altman limits of agreement were calculated. The correlation between AP diameter and MRI was assessed by calculating the Pearson correlation coefficient. Interobserver and intraobserver variability was assessed by calculating the absolute differences expressed as a percentage of the mean of repeated measurements. A two-tailed probability value of 0.05 or less was considered statistically significant and the $95 \%$ confidence intervals are presented. 
CHAPTER 4

\section{RESULTS}

Patient Population

Patient baseline characteristics are presented in Table 1.

Table 1 Patient baseline characteristics $(n=60)$

\begin{tabular}{ll}
\hline Variable & Value \\
\hline Men & $70 \%$ \\
Age $(\mathrm{yrs})$ & $61 \pm 10(32-77)$ \\
$\mathrm{BMI}\left(\mathrm{kg} / \mathrm{m}^{2}\right)$ & $27 \pm 5(19-40)$ \\
Left ventricular ejection fraction (\%) & $55 \pm 8(35-75)$ \\
Cardiovascular risk factors & \\
Obesity (BMI > $\left.30 \mathrm{~kg} / \mathrm{m}^{2}\right)$ & $22 \%$ \\
Smoking & $12 \%$ \\
Diabetes mellitus & $5 \%$ \\
Hypertension & $57 \%$ \\
Dyslipidemia & $45 \%$ \\
Positive family history & $30 \%$ \\
Type of atrial fibrillation & \\
Paroxysmal & $68 \%$ \\
Persistent & $32 \%$ \\
\hline
\end{tabular}

BMI, Body mass index. Data are expressed as mean \pm SD (range) or as percentages

Image Acquisition

Heart rates during acquisition did not differ significantly between echocardiography ( $67 \pm 16 \mathrm{bpm}$, range $42 \mathrm{bpm}-110 \mathrm{bpm}$ ) and MRI (63 $\pm 13 \mathrm{bpm}$, range $40 \mathrm{bpm}-97 \mathrm{bpm}$ ). Mean frame rates during echocardiographic image acquisition were $50 \pm 4 \mathrm{~Hz}$ (range 39$69 \mathrm{~Hz}$ ) for $2 \mathrm{DE}$ and $26 \pm 4 \mathrm{~Hz}$ (range $16-37 \mathrm{~Hz}$ ) for RT3DE. All datasets were of evaluable quality and were used for analysis and comparison. Fifty-three patients had the same rhythm during both echocardiographic and MRI data acquisitions, while 7 patients showed different rhythms during acquisitions (i.e. 6 patients were in sinus rhythm during echocardiography but in atrial fibrillation during MRI and 1 patient was in atrial fibrillation during echocardiography but in atrial fibrillation during MRI).

\section{Left Atrial Volumes and Correlation of Echocardiography with MRI}

Mean indexed LA volumes as derived by each method are depicted in Table 2. Intraclass correlation coefficients for $\mathrm{LA}_{\max }$ were 0.94 (95\% confidence interval $[\mathrm{Cl}] 0.88-0.97$, 
$p<0.001)$ for 4D LA Analysis versus MRI, 0.80 (95\% Cl $0.21-0.93, p<0.001)$ for QLAB versus MRI, $0.70(95 \% \mathrm{Cl} 0.46-0.83, \mathrm{p}<0.001)$ for Simpson versus $\mathrm{MRI}, 0.69(95 \% \mathrm{Cl}$ $0.42-0.83, p<0.001)$ for $A L$ versus $M R I$, and $0.24(95 \% \mathrm{Cl} 0.09-0.57, p<0.001)$ for $\mathrm{PE}$ versus MRI. For $L A_{\min }$, intraclass correlation analysis revealed coefficients of 0.95 ( $95 \%$ $\mathrm{Cl} 0.87-0.98, \mathrm{p}<0.001)$ for 4D LA Analysis versus MRI, 0.90 (95\% Cl $0.64-0.96$, $\mathrm{p}<0.001)$ for $\mathrm{QLAB}$ versus MRI, $0.83(95 \% \mathrm{Cl} 0.74-0.90, \mathrm{p}<0.001)$ for Simpson versus $\mathrm{MRI}, 0.82$ (95\% Cl $0.71-0.89, \mathrm{p}<0.001)$ for AL versus $\mathrm{MRI}$, and 0.36 (95\% Cl $0.09-0.67$, $\mathrm{p}<0.001$ ) for PE versus MRI. When comparing the AP diameter with MRI, Pearson correlation coefficients were found to be $0.63(95 \% \mathrm{Cl} p>0.001)$ for $L A_{\max }$, and $0.64(p<0.001)$ for $L A_{\min }$. Intraclass correlation coefficients for 4D LA Analysis volumes were significantly higher than in all other methods, while volumes derived from PE correlated significantly less (Figure 4).

Table 2. Indexed LA volumes ( $n=60)$

\begin{tabular}{|c|c|c|c|c|c|c|}
\hline Volume & MRI & 4D LA Analysis & QLAB & $\begin{array}{l}\text { Biplane } \\
\text { Simpson's rule }\end{array}$ & $\mathrm{AL}$ & PE \\
\hline $\operatorname{La}_{\max }\left(\mathrm{ml} / \mathrm{m}^{2}\right)$ & $58 \pm 17(27-106)$ & $56 \pm 16(27-106)$ & $49 \pm 15(26-96)$ & $51 \pm 15(26-94)$ & $51 \pm 15(24-91)$ & $31 \pm 10(13-57)$ \\
\hline $\operatorname{La}_{\max }\left(\mathrm{ml} / \mathrm{m}^{2}\right)$ & $40 \pm 19(13-102)$ & $36 \pm 7(13-101)$ & $34 \pm 17(12-91)$ & $38 \pm 15(14-76)$ & $37 \pm 15(11-75)$ & $21 \pm 9(5-53)$ \\
\hline
\end{tabular}

Data are expressed as mean \pm SD (range).

Compared to $\mathrm{MRI}$, Bland-Altman analysis for $L A_{\max }$ revealed limits of agreement of -29 $\mathrm{ml}$ to $19 \mathrm{ml}$ for 4D LA Analysis, $-49 \mathrm{ml}$ to $15 \mathrm{ml}$ for QLAB, -63 $\mathrm{ml}$ to $36 \mathrm{ml}$ for Simpson, $65 \mathrm{ml}$ to $36 \mathrm{ml}$ for $\mathrm{AL}$, and $-109 \mathrm{ml}$ to $3 \mathrm{ml}$ for PE. Similarly, limits of agreement for $L A_{\min }$ were $-26 \mathrm{ml}$ to $13 \mathrm{ml}$ for $4 \mathrm{D}$ LA Analysis, $-37 \mathrm{ml}$ to $15 \mathrm{ml}$ for QLAB, $-44 \mathrm{ml}$ to $38 \mathrm{ml}$ for Simpson, $-48 \mathrm{ml}$ to $38 \mathrm{ml}$ for $\mathrm{AL}$, and $-92 \mathrm{ml}$ to $19 \mathrm{ml}$ for PE (Figure 5). Thus, for $95 \%$ of measurements, percent difference from MRI measurements can be expected to be within $28 \%$ of the reference value for 4D LA Analysis, 37\% for QLAB, 49\% for Simpson, $52 \%$ for $\mathrm{AL}$, and within $68 \%$ for PE. On the other hand, for $4 \mathrm{D}$ LA Analysis $54 \%$ of measurements are within $10 \%$ of the reference value. For QLAB this can be expected in $42 \%$ of measurements, for Simpson and AL in $23 \%$. By contrast, for the PE method only $4 \%$ of measurements fall within the $10 \%$ range. 

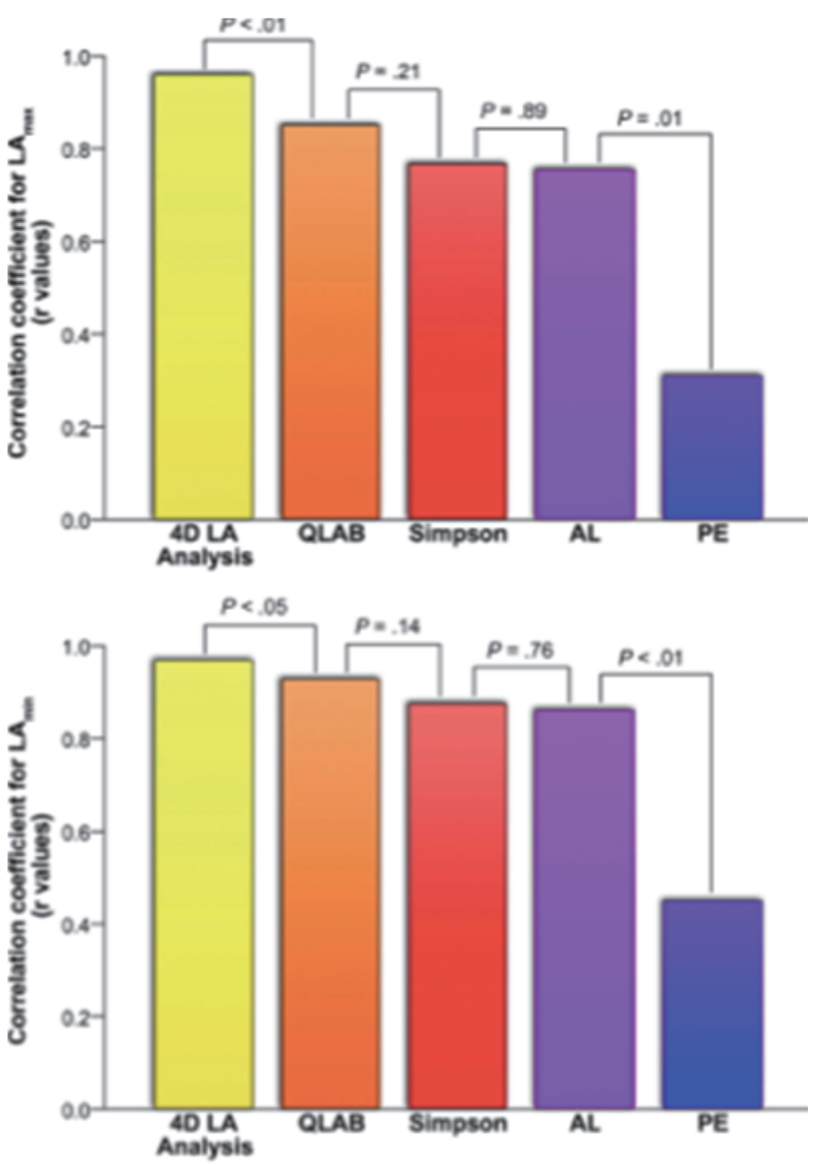

Figure 4. Intraclass correlation with magnetic resonance imaging of maximum (left) and minimum (right) left atrial volumes acquired by different eohocardiographic methods. Correlation coefficients for 4D LA Analyses and prolate ellipsoide method differed significantly from the coefficients of all other echocardiographic metlhods.

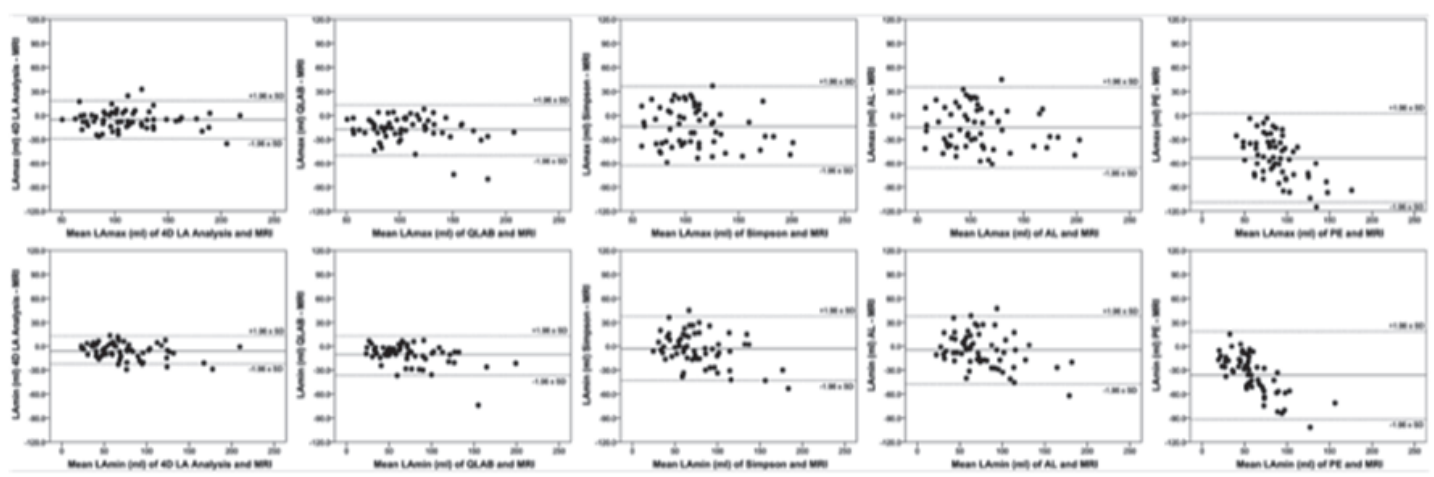

Figure 5. Bland-Altman plots comparing maximum (top row) and minimum (bottom row) left atrial volume measurements obtained by different echocardiographic methods and MRI serving as reference. 
Of note, when analyzed separately, there was no significant difference when comparing intraclass correlations between the patients who had the same heart rhythm during both echocardiography and MRI and patients who did not.

Intraclass correlations coefficients and percentage differences from the mean for intraobserver repeat measurements for maximum volumes were $r=0.97$ and $+2.8 \%$ for 4D LA Analysis, $r=0.99$ and $-0.8 \%$ for $Q L A B, r=0.96$ and $+3.6 \%$ for Simpson, $r=0.95$ and $0.7 \%$ for $A L$, and $r=0.95$ and $+3.4 \%$ for $P E$. For $L A_{\min }$, they were $r=0.98$ and $+1.0 \%$ for $4 D$ LA Analysis, $r=0.99$ and $+4.0 \%$ for QLAB, $r=0.98$ and $+2.4 \%$ for the biplane Simpson's method, $r=0.98$ and $+7.0 \%$ for $A L$, and $r=0.95$ and $-0.4 \%$ for PE. For interobserver repeat measurements, intraclass correlation coefficients and percentage differences from the mena for $L A_{\max }$ were $r=0.96$ and $-4.3 \%$ for $4 D$ LA Analysis, $r=0.92$ and $-5.5 \%$ for $Q L A B$, $r=0.88$ and $-9.0 \%$ for the biplane Simpson's method, $r=0.889$ and $-6.8 \%$ for $A L$, and $r=0.91$ and $+2.4 \%$ for PE. For LAmin, they were $r=0.94$ and $-5.4 \%$ for 4D LA Analysis, $r=$ 0.89 and $-2.4 \%$ for QLAB, $r=0.92$ and $-7.2 \%$ for the biplane Simpson's method, $r=0.89$ and $-5.3 \%$ for $\mathrm{AL}$, and $\mathrm{r}=0.95$ and $-6.8 \%$ for $\mathrm{PE}$.

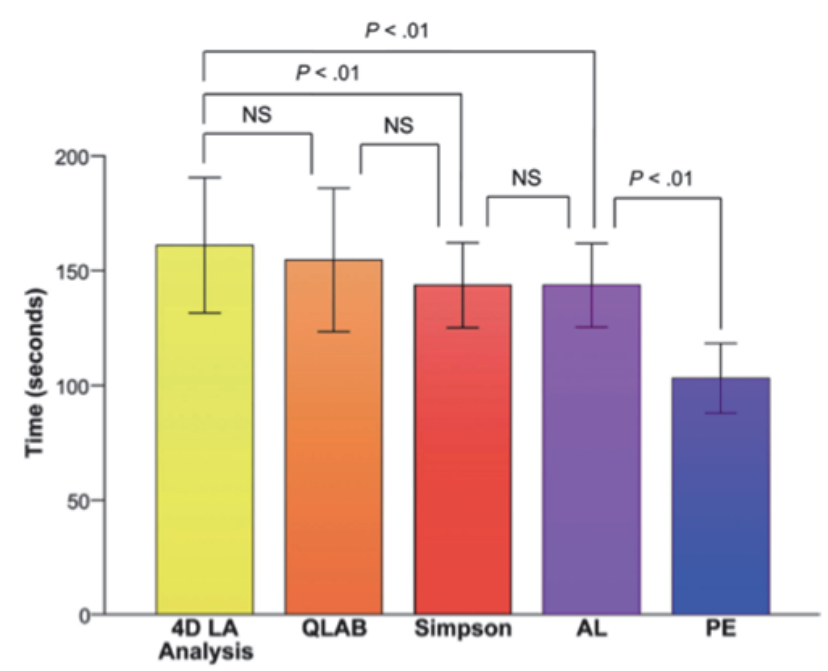

Figure 6. Comparison of analysis time associated with the use of different methods for offline calculation of maximum and minimum echocardiographic left atrial volumes.

\section{Echocardiographic Analysis Time}

The required amount of time for offline LA volume calculation was $161 \pm 29$ seconds (range 84 - 227) for 4D LA Analysis, 155 \pm 31 seconds (range 81 - 226) for QLAB, 144 \pm 19 seconds (range 97 - 190) for Simpson, $144 \pm 18$ seconds (range 102 - 196) for AL, and $103 \pm 15$ seconds (range 69 - 144) for PE. LA volume analysis using PE was significantly shorter than for all other methods. On the contrary, 4D LA analysis and QLAB both required significantly more time for analysis than Simpson and $A L$, which were equally time consuming (Figure 6). 


\section{DISCUSSION}

Although previous studies have compared different echocardiographic methods for LA quantification with each other ${ }^{4,5,16-18}$ or a single echocardiographic method with cardiac $M R I^{3,6,8}$ the present study represents the first head-to-head comparison of all commonly available and routinely used 2DE and novel RT3DE methods and provides comprehensive data in terms of accuracy and feasibility by using cardiac MRI as the standard of reference and by providing information on analysis time for offline analysis associated with each method. In terms of accuracy, a novel RT3DE analysis tool (i.e., 4D LA Analysis) dedicated specifically to the assessment of the left atrium was correlated significantly better with MRI than all other echocardiographic methods, while the second RT3DE analysis tool originally developed for the analysis of the left ventricle (i.e., QLAB) performed slightly (but not significantly) better than the biplane modified Simpson's rule and AL. By contrast, LA volumes as assessed using PE were correlated substantially less with the findings from MRI. Similar to the correlation analysis, Bland-Altman analysis revealed the narrowest limits of agreement for the 4D LA Analysis software tool, while the limits of agreement grew wider with the other methods. Of note, BlandAltman analysis of PE revealed a massive underestimation of volumes, particularly in severely enlarged atria, while all other methods showed slight, but consistent underestimations of both $L A_{\min }$ and $L A_{\max }$ throughout the entire range. $A$ likely explanation that has been proposed for this finding is the disproportionately larger change in volume

that is associated with a small increase in a single major LA axis, ${ }^{5,17,24,25}$ thus leading to volume underestimation in methods assuming a consistent relation to other LA dimensions, which may be particularly true for PE. In pathologic conditions, the left atrium dilates to a larger extent in the longitudinal and anterior-posterior dimensions compared to the mediolateral dimension. This asymmetric dilation may more closely be accounted for with RT3DE methods, as these rely on anatomic contouring of the LA endocardium in all dimensions, thus leading to more accurate and consistent volumes than with 2D methods, which may be insensitive to such nonuniform changes in spatial LA configuration. Furthermore, the higher spatial resolution of MRI in comparison with echocardiography has been repeatedly suggested as a likely explanation for the general underestimation of LA volumes by echocardiography. ${ }^{6}$ As a consequence, echocardiography cannot for example distinguish volumes within intratrabecular atrial areas. Additionally, the apical four-chamber view places the left atrium at the far field of the ultrasound beam, resulting in an additional loss of image resolution.

Although these novel RT3DE methods enable more accurate LA volume quantification, they require more elaborate contouring and manual refinement of the semiautomatic endocardial border detection than in traditional 2DE methods, especially in lowquality data sets. In the present study, analysis time associated with offline analysis was slightly higher for 4D LA Analysis compared with 2DE analysis. However, although our data do not include the additional effort of RT3DE image acquisition itself and thus may 
underestimate differences, the additional time needed for analysis may potentially be compensated by additional functional parameters that accompany RT3DE LA analysis, such as information on segmental atrial wall motion, the LA active contraction component, and even dyssynchrony parameters. Furthermore, recent developments applying fully automated endocardial contour detection to obtain left ventricular volumes from RT3DE imaging ${ }^{26}$ may eventually be adopted for LA analysis in the near future, ${ }^{27}$ potentially leading to a further substantial reduction in analysis time. This in turn may provide the basis for a more widespread use of RT3DE methods for LA volume quantification in the clinical arena. However, further studies are needed to assess the prognostic value of overall and in particularly for regional LA functional parameters in various conditions to justify the additional effort of LA assessment using RT3DE imaging and to fully reveal its presumed clinical potential in the near future.

It may be perceived as a limitation that the present study evaluated patients referred for pulmonary vein isolation rather than a normal population. Thus, as expected, a majority of patients presented with dilated left atria, and we cannot therefore conclusively comment on the number of subjects being correctly classified as having normalsized atria. However, this fact may also be perceived as a strength of the present study, because it allowed us to include a broader range of volumes in the comparison.

Correlation coefficients for RT3DE imaging using QLAB and for the 2D techniques were notably smaller than in previous reports comparing echocardiographic methods for the left atrium with MRI. ${ }^{3,4}$ This may be because of different inclusion criteria; in the present study, LA volumes were substantially larger than in previous works because we included patients referred for pulmonary vein isolation. This may have led to lower agreement with MRI, particularly for large volumes. Furthermore, we applied intraclass correlation analysis rather than simple linear regression analysis, which does not appropriately reflect systematic underestimation or overestimation of volumes.

Furthermore, the time required for RT3DE analysis using QLAB was substantially higher in the present study than reported in a previous work ${ }^{6}(155 \pm 31$ vs $56 \pm 8 \mathrm{sec}$ ). In our experience, the most time consuming part of LA analysis by RT3DE imaging consists of manual recontouring and optimization of the contours after applying automated border detection algorithms. Such manual recontouring may not have been performed in previous work, leading to reduced comparability of analysis times.

Finally, the fact that approximately one third of patients were in atrial fibrillation during retrospectively gated MRI and/or RT3DE image acquisition may have led to decreased image quality, which may in turn have had an impact on exact endocardial border delineation. We cannot comment with certainty on the impact this may have had on the calculated volumes in terms of overestimation or underestimation, because there is no noninvasive imaging gold standard for the determination of LA volumes that is independent of cardiac rhythm. However, the fact that intraclass correlation coefficients for patients who had different cardiac rhythms during echocardiography and MRI did not differ significantly from those for patients who had the same cardiac rhythm for 


\section{CHAPTER 4}

both imaging techniques suggests that cardiac rhythm had only a limited impact on the measured LA volumes.

\section{Conclusions}

Commonly used biplane methods from conventional 2D echocardiography (i.e., the biplane modified Simpson's rule and AL) offer reasonable accuracy for LA chamber quantification across a broad range of volumes, while RT3DE methods lead to a moderate improvement in accuracy at the cost of more time needed for offline analysis.

\section{Acknowledgements}

We acknowledge that a vast majority of the patient population analyzed in the present study is shared with another scientific article published in a different journal ${ }^{28}$. Whereas the present work focuses on comparison of various 2DE and RT3DE methods for LA volume analysis with MRI serving as standard of reference, the aforementioned study's aim was the validation of the RT3DE software analysis tool 4D LA Analysis, in particular its ability to assess the active component of atrial ejection. Of note, the same RT3DE and MRI acquisitions used in the present study were also included in the other study. However, offline analysis of raw data sets was separately and independently performed for both works. Thus, although certain methodologic aspects are identical for both, the studies' backgrounds, results, scope, and conclusions differ substantially. 


\section{REFERENCES}

1. Lang RM, Bierig M, Devereux RB, Flachskampf FA, Foster E, Pellikka PA, et al. Recommendations for chamber quantification: a report from the American Society of Echocardiography's Guidelines and Standards Committee and the Chamber Quantification Writing Group, developed in conjunction with the European Association of Echocardiography, a branch of the European Society of Cardiology. J Am Soc Echocardiogr. 2005;18:1440-63.

2. Tsang TS, Abhayaratna WP, Barnes ME, Miyasaka Y, Gersh BJ, Bailey KR, et al. Prediction of cardiovascular outcomes with left atrial size: is volume superior to area or diameter? J Am Coll Cardiol. 2006;47:1018-23.

3. Keller AM, Gopal AS, King DL. Left and right atrial volume by freehand three-dimensional echocardiography: in vivo validation using magnetic resonance imaging. Eur J Echocardiogr. 2000;1:55-65.

4. Jenkins C, Bricknell K, Marwick TH. Use of real-time three-dimensional echocardiography to measure left atrial volume: comparison with other echocardiographic techniques. J Am Soc Echocardiogr. 2005;18:991-7.

5. Maddukuri PV, Vieira ML, DeCastro S, Maron MS, Kuvin JT, Patel AR, et al. What is the best approach for the assessment of left atrial size? Comparison of various unidimensional and two-dimensional parameters with three-dimensional echocardiographically determined left atrial volume. J Am Soc Echocardiogr. 2006;19:1026-32.

6. Artang R, Migrino RQ, Harmann L, Bowers M, Woods TD. Left atrial volume measurement with automated border detection by 3-dimensional echocardiography: comparison with Magnetic Resonance Imaging. Cardiovasc Ultrasound. 2009;7:16.

7. Miyasaka Y, Tsujimoto S, Maeba H, Yuasa F, Takehana K, Dote K, et al. Left atrial volume by real-time three-dimensional echocardiography: validation by 64-slice multidetector computed tomography. J Am Soc Echocardiogr. 2011;24:680-6.

8. Rohner A, Brinkert M, Kawel N, Buechel RR, Leibundgut G, Grize L, et al. Functional assessment of the left atrium by real-time three-dimensional echocardiography using a novel dedicated analysis tool: initial validation studies in comparison with computed tomography. Eur J Echocardiogr. 2011;12:497-505.

9. Benjamin EJ, D'Agostino RB, Belanger AJ, Wolf PA, Levy D. Left atrial size and the risk of stroke and death. The Framingham Heart Study. Circulation. 1995;92:835-41.

10. Matsuda M, Matsuda Y. Mechanism of left atrial enlargement related to ventricular diastolic impairment in hypertension. Clin Cardiol. 1996;19:954-9.

11. Modena MG, Muia N, Sgura FA, Molinari R, Castella A, Rossi R. Left atrial size is the major predictor of cardiac death and overall clinical outcome in patients with dilated cardiomyopathy: a long-term follow-up study. Clin Cardiol. 1997;20:553-60.

12. Tsang TS, Barnes ME, Gersh BJ, Bailey KR, Seward JB. Left atrial volume as a morphophysiologic expression of left ventricular diastolic dysfunction and relation to cardiovascular risk burden. Am J Cardiol. 2002;90:1284-9.

13. Moller JE, Hillis GS, Oh JK, Seward JB, Reeder GS, Wright RS, et al. Left atrial volume: a powerful predictor of survival after acute myocardial infarction. Circulation. 2003;107:2207-12.

14. Osranek M, Fatema K, Qaddoura F, Al-Saileek A, Barnes ME, Bailey KR, et al. Left atrial volume predicts the risk of atrial fibrillation after cardiac surgery: a prospective study. J Am Coll Cardiol. 2006;48:779-86.

15. Suh IW, Song JM, Lee EY, Kang SH, Kim MJ, Kim JJ, et al. Left atrial volume measured by real-time 3dimensional echocardiography predicts clinical outcomes in patients with severe left ventricular dysfunction and in sinus rhythm. J Am Soc Echocardiogr. 2008;21:439-45.

16. Rodevan O, Bjornerheim R, Ljosland M, Maehle J, Smith HJ, Ihlen H. Left atrial volumes assessed by threeand two-dimensional echocardiography compared to MRI estimates. Int J Card Imaging. 1999;15:397410.

17. Jiamsripong P, Honda T, Reuss CS, Hurst RT, Chaliki HP, Grill DE, et al. Three methods for evaluation of left atrial volume. Eur J Echocardiogr. 2008;9:351-5. 


\section{CHAPTER 4}

18. Russo C, Hahn RT, Jin Z, Homma S, Sacco RL, Di Tullio MR. Comparison of echocardiographic single-plane versus biplane method in the assessment of left atrial volume and validation by real time threedimensional echocardiography. J Am Soc Echocardiogr. 2010;23:954-60.

19. Kircher B, Abbott JA, Pau S, Gould RG, Himelman RB, Higgins CB, et al. Left atrial volume determination by biplane two-dimensional echocardiography: validation by cine computed tomography. Am Heart J. 1991;121:864-71.

20. Jarvinen VM, Kupari MM, Hekali PE, Poutanen VP. Right atrial MR imaging studies of cadaveric atrial casts and comparison with right and left atrial volumes and function in healthy subjects. Radiology. 1994;191:137-42.

21. Vandenberg BF, Weiss RM, Kinzey J, Acker M, Stark CA, Stanford W, et al. Comparison of left atrial volume by two-dimensional echocardiography and cine-computed tomography. Am J Cardiol. 1995;75:754-7.

22. Childs H, Ma L, Ma M, Clarke J, Cocker M, Green J, et al. Comparison of long and short axis quantification of left ventricular volume parameters by cardiovascular magnetic resonance, with ex-vivo validation. J Cardiovasc Magn Reson 2011;13:40.

23. Dunn OJ, Clark V. Comparison of Tests of the Equality of Dependent Correlation Coefficients. J Am Stat Assoc. 1971;66:904.

24. Schabelman S, Schiller NB, Silverman NH, Ports TA. Left atrial volume estimation by two-dimensional echocardiography. Cathet Cardiovasc Diagn. 1981;7:165-78.

25. Lester SJ, Ryan EW, Schiller NB, Foster E. Best method in clinical practice and in research studies to determine left atrial size. Am J Cardiol. 1999;84:829-32.

26. Thavendiranathan P, Liu S, Verhaert D, Calleja A, Nitinunu A, Van Houten T, et al. Feasibility, Accuracy, and Reproducibility of Real-Time Full-Volume 3D Transthoracic Echocardiography to Measure LV Volumes and Systolic Function: A Fully Automated Endocardial Contouring Algorithm in Sinus Rhythm and Atrial Fibrillation. JACC Cardiovasc Imaging. 2012;5:239-51.

27. Hung J, Lang R, Flachskampf F, Shernan SK, McCulloch ML, Adams DB, et al. 3D echocardiography: a review of the current status and future directions. J Am Soc Echocardiogr. 2007;20:213-33.

28. Buechel RR, Sommer G, Leibundgut G, Rohner A, Riede F, Kessel-Schaefer A, et al. Assessment of left atrial functional parameters using a novel dedicated analysis tool for real-time three-dimensional echocardiography: validation in comparison to magnetic resonance imaging. Int J Cardiovasc Imaging. 2012. 


\section{CHAPTER 5}

Improvement in left ventricular ejection fraction and reverse remodelling in elderly heart failure patients on intense NT-proBNPguided therapy

Beat A. Kaufmann MD, Kaatje Goetschalckx MD, Son Y. Min MD, Micha T. Maeder MD, Urs Bucher MD, Fabian Nietlispach MD, Alain M. Bernheim MD, Matthias E. Pfisterer MD, Hans-Peter Brunner-La Rocca MD

Int J Cardiol. 2015;191:286-293 


\section{CHAPTER 5}

\section{ABSTRACT}

Background: In chronic heart failure, left ventricular ejection fraction (LVEF) is considered to be stable. Intensified therapy may improve survival, but little is known whether this is associated with reverse remodelling and dependent on age and NT-proBNP guidance. We aimed to define the evolution of LVEF under intensified therapy in relation to age and NT-proBNP guidance.

Methods and Results: Echocardiography was performed at baseline, 12 and 18 months in TIME-CHF, a trial comparing NT-proBNP versus symptom-guided therapy in patients aged 60 to 74 and $\geq 75$ years. LVEF, LV end diastolic volume index (LVEDVI) and end systolic volume index (LVESVI) were assessed. LVEF increased from $31 \pm 11 \%$ to $40 \pm 12 \%$ at 12 ( $p<0.001)$, and $42 \pm 13 \%$ at 18 months ( $p=0.004$ vs 12 months). The increase in LVEF was significantly larger in the NT-proBNP-guided treatment group ( $p$ for interaction $=0.006$ ). LVEDVI decreased from $88 \pm 34 \mathrm{ml} / \mathrm{m}^{2}$ to $77 \pm 39 \mathrm{ml} / \mathrm{m}^{2}$ and $70 \pm 33 \mathrm{ml} / \mathrm{m}^{2}$ $(p<0.001)$, as did LVESVI $(p<0.001)$, without influence by study group allocation.

Conclusions: In elderly heart failure patients, intensified medical therapy leads to an improvement in LVEF and to reverse remodeling. NT-proBNP guided therapy was associated with a larger improvement in LVEF than symptom guided therapy, independent of age. 


\section{INTRODUCTION}

In patients with heart failure and a left ventricular ejection fraction (LVEF) below 45\%, prognosis is related to left ventricular remodeling ${ }^{1,2}$. Angiotensin-converting enzyme inhibitors, betablockers, mineralocorticoid antagonists, cardiac resynchronization therapy, and ivabradine have all been shown to either slow the decrease or to lead to a modest improvement of LVEF with only resynchronization therapy showing a larger effect, albeit in a more selected patient population ${ }^{3-8}$. All of these studies have been conducted in patients with an average age $<70$ years, whereas the majority of patients with heart failure in the general population are older ${ }^{9}, 10$. Once medical therapy has been established in chronic heart failure, LVEF is considered to be relatively stable, but data from studies prospectively evaluating changes in LVEF over time are scarce. LVEF and left ventricular volumes have been examined in the PROTECT trial over time ${ }^{11,12}$, but this was a smaller trial and the patients included were on average 10 years younger than in the general heart failure population.

The Trial of Intensified versus standard Medical therapy in Elderly patients with Congestive Heart Failure (TIME-CHF) provided an excellent opportunity to investigate if LVEF remains stable on chronic heart failure therapy also in elderly patients comparable to the general heart failure population and whether intensified medical therapy could also lead to improvements in LVEF and reverse remodelling. TIME CHF compared a NTproBNP-guided, intensified treatment strategy with a standard symptom-guided therapy in patients aged 75 years or older compared with patients aged 60 to 74 years ${ }^{13}$. It showed that younger patients benefitted from intensified heart failure therapy regarding survival in contrast to patients $>75$ years of age. Reasons for this age difference remained unclear. It is to note, that all patients were on standard heart failure medication at baseline, but drug therapy and doses were significantly increased during the course of the study. This change in therapy differed between the two groups with patients allocated to NT-proBNP guided therapy receiving more intense therapy. Thus, the questions came up, whether a more intense heart failure therapy would be associated with a greater change in LV function and reverse remodeling and whether this was different between the two age groups. Accordingly, the specific aims of this echocardiographic substudy of the TIME-CHF trial were (a) to evaluate the effect of intensified NT-proBNP-guided versus symptom-guided heart failure therapy on LVEF and left ventricular remodeling, (b) to assess the effect of age on these parameters and (c) to investigate the impact of changes in LVEF on outcome. 


\section{CHAPTER 5}

\section{METHODS}

\section{Study population and trial design}

TIME-CHF was a multicenter trial comparing an intensified, NT-proBNP-guided treatment strategy with a symptom-guided treatment strategy in patients aged 60 years or more with heart failure irrespective of LVEF. The design of the TIME-CHF trial has been described elsewhere in detail ${ }^{13,14}$. Briefly, patients with dyspnea (New York Heart Association class II or higher on current therapy), a history of hospitalization for heart failure within the past year, and an elevated NT-proBNP level $(>400 \mathrm{pg} / \mathrm{ml}$ in patients $<75$ years, and $>800 \mathrm{pg} / \mathrm{ml}$ in patients $\geq 75$ years of age) were recruited in 15 tertiary and secondary hospital centers in Switzerland and Germany. Exclusion criteria were dyspnea not mainly due to heart failure, valvular heart disease requiring surgery, acute coronary syndrome within ten days before study inclusion, angina pectoris $\geq \operatorname{CCS} 2$, revascularization within the month before study inclusion, body mass index $>35$, serum creatinine $>2.49 \mathrm{mg} / \mathrm{dL}$, a life expectancy of less than 3 years due to non-cardiovascular causes, inability to give informed consent, follow-up impossible, or participation in another study. All patients gave written informed consent. The study was approved by the local ethics committees of each participating center. Overall, 499 patients with a systolic dysfunction defined as an LVEF of $\leq 45 \%$ were included in the original TIME-CHF trial and were eligible for the present substudy.

Patients were stratified into two pre-specified age groups (60-74 years, and $\geq 75$ years) and then randomized to either symptom-guided therapy or NT-proBNP-guided therapy. Patients, but not the treating physicians were blinded for treatment allocation. Patients were evaluated clinically at baseline, and after 1, 3, 6, 12, and 18 months. At baseline, a medical history together with clinical signs of heart failure and vital signs was recorded. The duration of heart failure was estimated from the patient history and categorized as up to 2 months, $>2$ months up to 12 months, and $>12$ months. NTproBNP was measured centrally at the University Hospital of Basel with a standard assay kit (Roche Diagnostics, Switzerland). Results were made available to the treating physicians for all patients at baseline, and thereafter only for patients allocated to intensified therapy. Medical treatment in patients randomized to the symptom guided group was adjusted with the goal of reducing symptoms to NYHA $\leq I$, whereas in patients randomized to the NT-proBNP group, treatment was adjusted with the goal of reducing NTproBNP levels to $<400 \mathrm{pg} / \mathrm{ml}$ in patients aged $60-74$ years, and $<800 \mathrm{pg} / \mathrm{ml}$ in patients aged $\geq 75$, and symptoms to NYHA $\leq 1$. Treatment escalation followed standardized protocols ${ }^{14}$ up to 12 months. 


\section{Echocardiography}

Patients had transthoracic echocardiography performed at the recruiting center by a board certified cardiologist trained in echocardiography. Standard clinical ultrasound equipment was used for acquisition of cine loops documenting left ventricular function and dimensions from parasternal and apical acoustic windows with broadband transducers operating in harmonic imaging mode. The echocardiographic studies were stored digitally and transferred to the echocardiography core laboratory at the University Hospital of Basel. The studies were read at the core laboratory by 2 trained cardiologists (S.-Y. M., K.G.) blinded to treatment allocation. Apical four chamber and apical two chamber views were used to derive LVEF, left ventricular enddiastolic volume index (LVEDVI) and left ventricular endsystolic volume index (LVESVI) using the biplane Simpson's method according to the recommendation of the American Society of Echocardiography ${ }^{15}$. In patients who had only an apical four chamber view available for analysis at either of the visits, the apical four chamber view was used for analysis at all timepoints. Patients, in whom 4 or more segments of the 17-segment LV model (ASE segmental model) showed an insufficient delineation of the endocardial border, were excluded from the analysis. The left atrial antero-posterior diameter (LA) and tricuspid annular plane systolic excursion (TAPSE) were measured according to current guidelines 16. Transmitral inflow velocities and left ventricular outflow tract velocities were obtained from pulsed wave Doppler tracings with the sample volume placed between the tips of the mitral valve leaflets and in the left ventricular outflow tract, respectively. The right ventricular-right atrial pressure gradient was obtained from the peak tricuspid regurgitation jet velocity.

Changes in the measured echocardiographic parameters were examined over time and according to age and NT-proBNP-guided versus symptom-guided treatment. For the purpose of assessing the effect of changes in LVEF on clinical outcome, patients with complete echocardiographic datasets at 0 and 12 months were divided into patients with an increase in LVEF by $\geq 10$ absolute percentage points and into patients with a smaller increase or a decrease in LVEF. This cut-off was selected based on previous data indicating 10 percentage points as the upper limit of temporal variability of twodimensional measurements of ejection fraction ${ }^{17}$.

\section{Statistics}

Statistical analyses were performed using SPSS Version 21.0 (SPSS Inc.). Results are shown as frequencies or means $\pm 1 S D$, unless noted otherwise. Between group comparisons were performed using a t-test or a Mann-Whitney test, as appropriate. A general linear model for repeated measures with Bonferroni-corrected post hoc tests was used to assess changes over time. General linear models with Pillai's trace test were used for assessing the influence of age and group assignment on LVEF over time. Kaplan-Meier 


\section{CHAPTER 5}

curves were used for assessing time dependent occurrence of events in these two patient groups. The log-rank test was used to compare the occurrence of endpoints between groups. A two-sided $p$ value $<0.05$ was considered statistically significant.

\section{RESULTS}

\section{Patient characteristics}

Of 499 patients eligible for the present substudy, 2 were lost to follow up and 59 withdrew consent before completing the 18 months follow up. Among the remaining 438 patients, 84 died within the first 12 months after study entry, and another 17 died between 12 and 18 months after study entry. Of the 337 patients available for comparison at 0,12 and 18 months, 168 (49.9\%, n=60 aged 60-74 years, and $n=108$ aged $\geq 75)$ had complete, evaluable echocardiographic datasets at all three time-points; these 168 patients comprise the main study population reported here.

Ancillary analyses were also conducted in 188 patients (55.7\% of those eligible) who had complete echocardiographic datasets at 0 and 18 months, and in 233 patients (65.8\% of those eligible) who had complete echocardiographic datasets at 0 and 12 months.

The baseline characteristics of the 168 patients included in the main study population are shown in table 1 in comparison with baseline characteristics of the remaining patients of the original TIME-CHF study $(n=331)$. Among patients within the reported main study population of the present report there were no significant differences both between the symptom-guided and the NT-proBNP-guided study groups nor did these patients differ from the total TIME-CHF study population with reduced LVEF except for fewer male patients in the main population of the present report.

\section{Left ventricular ejection fraction}

In 168 patients who had evaluable images at all three time-points, LVEF significantly increased during the study period from $30.8 \pm 11.2 \%$ to $39.7 \pm 12.3 \%$ from 0 to 12 months and further to $41.6 \pm 12.7 \%$ from 12 to 18 months (Figure 1A). The increase in LVEF was not influenced by age $(p=0.091$ for interaction; increase from $29.4 \pm 11.7 \%$ to $42.6 \pm 12.1 \%$ in patients aged $60-74$ years, and from $31.5 \pm 10.9 \%$ to $41.1 \pm 13.1 \%$ from 0 to 18 months in patients $\geq 75$ years). Also, the changes in LVEF were independent of the prior duration of heart failure with patients with less than 2 months history of heart failure increasing from an LVEF of $31.4 \pm 11.9 \%$ at baseline to $41.2 \pm 13.4 \%$ at 18 months, patients with 2-12 months 
Table 1. Baseline characteristics of the substudy population and comparison with the population of the TIMECHF main study.

\begin{tabular}{|c|c|c|c|}
\hline & \multicolumn{2}{|l|}{$\begin{array}{l}\text { Included } \\
(n=168)\end{array}$} & \multirow{2}{*}{$\begin{array}{l}\text { Not Included } \\
(n=331) \\
\text { Main TIME CHF Study } \\
(n=331)\end{array}$} \\
\hline & $\begin{array}{l}\text { Symptom guided } \\
(n=81)\end{array}$ & $\begin{array}{l}\text { NT-proBNP-guided } \\
(\mathrm{n}=87)\end{array}$ & \\
\hline Age (years) & $76.8 \pm 7.8$ & $76.6 \pm 7.3$ & $75.8 \pm 7.5$ \\
\hline Male (\%) & $44(54) *$ & $56(64)$ & $227(69)$ \\
\hline Current smoking & $13(16)$ & $30(16)$ & $68(12)$ \\
\hline $\mathrm{BMI}(\mathrm{kg} / \mathrm{m} 2)$ & $24.9 \pm 4.2$ & $25.5 \pm 3.8$ & $25.4 \pm 4.2$ \\
\hline \multicolumn{4}{|l|}{ Cardiac parameters } \\
\hline Heart rate (bpm) & $77 \pm 17$ & $76 \pm 15$ & $75 \pm 14$ \\
\hline Systolic BP (mmHg) & $120 \pm 18$ & $118 \pm 19$ & $118 \pm 18$ \\
\hline Diastolic BP (mmHg) & $74 \pm 12$ & $70 \pm 11$ & $71 \pm 12$ \\
\hline eGFR (ml/min/1.73m2) & $54 \pm 16.0$ & $55 \pm 20$ & $51 \pm 22$ \\
\hline NT-proBNP (pg/ml) & $3723(1798-5648)$ & 3595 (1176-6014) & 4684 (1866-7502) \\
\hline \multicolumn{4}{|l|}{ NYHA class } \\
\hline Class II (\%) & $20(25)$ & $24(28)$ & $84(25)$ \\
\hline Class III (\%) & $52(64)$ & $54(62)$ & $200(60)$ \\
\hline Class IV (\%) & $9(11)$ & $9(10)$ & $47(14)$ \\
\hline \multicolumn{4}{|l|}{ Cause of heart failure } \\
\hline CAD (\%) & $44(54)$ & $44(51)$ & $199(60)$ \\
\hline DCM (\%) & $20(25)$ & $19(22)$ & $49(15)$ \\
\hline HHD (\%) & $14(17)$ & $22(25)$ & $71(22)$ \\
\hline VHD (\%) & $3(3.7)$ & $1(1)$ & $10(3)$ \\
\hline Diabetes & $26(32)$ & $27(31)$ & $119(36)$ \\
\hline Stroke & $9(11)$ & $13(15)$ & $54(16)$ \\
\hline Atrial fibrillation & $24(30)$ & $27(31)$ & $107(32)$ \\
\hline \multicolumn{4}{|l|}{ Medication } \\
\hline ACE-inhibitor & $67(83)$ & $65(75)$ & $260(79)$ \\
\hline $\begin{array}{l}\text { Angiotensin receptor } \\
\text { blocker }\end{array}$ & $10(12)$ & $13(15)$ & $59(18)$ \\
\hline $\begin{array}{l}\text { Renin-Angiotensin } \\
\text { blockade }\end{array}$ & $77(95)$ & $78(90)$ & $318(96)$ \\
\hline$\beta$-blocker & $65(80)$ & $71(82)$ & $256(77)$ \\
\hline Loop diuretics & $73(90)$ & $76(87)$ & $317(96)$ \\
\hline Spironolactone & $27(33)$ & $32(37)$ & $143(43)$ \\
\hline Nitrates & $30(37)$ & $30(34)$ & $83(25)$ \\
\hline Digoxin & $15(19)$ & $13(15)$ & $69(21)$ \\
\hline Statin & $42(52)$ & $37(43)$ & $156(47)$ \\
\hline Aspirin & $43(53)$ & $38(44)$ & $151(46)$ \\
\hline Oral anticoagulation & $42(52)$ & $49(56)$ & 194 (59) \\
\hline
\end{tabular}

Data are presented as numbers of patients (\%) or means $\pm 1 \mathrm{SD}$, except for NT-proBNP, where median and interquartile range is given. $\mathrm{BMI}$, body mass index; bpm, beats per minute; $\mathrm{BP}$, blood pressure; eGFR, estimated glomerular filtration rate, MDRD formula; CAD, coronary artery disease; DCM, dilated cardiomyopathy; VHD, valvular heart disease. ${ }^{*} p<0.05$ vs. patients not included in the study. 


\section{CHAPTER 5}

history of heart failure increasing from $31.3 \pm 13.4 \%$ to $43.9 \pm 13.2 \%$, and patients with $>12$ months history of heart failure increasing from $30.3 \pm 10.2 \%$ to $41.2 \pm 12.3 \%$ ( $p=0.92$ for the interaction of the duration of heart failure history with changes in LVEF).

Ancillary analyses performed in 188 patients with a complete dataset at 0 and 18 months confirmed the above results and are shown in table 2 .

Table 2. Evolution of left ventricular ejection fraction and left ventricular volumes in 188 patients ( $n=88$ symptom-guided, n=100 NT-proBNP-guided) with complete echocardiographic data at baseline and 18 months.

\begin{tabular}{|c|c|c|c|c|}
\hline & Baseline & 18 months & $\begin{array}{l}\mathrm{p} \text { baseline vs. } \\
18 \text { months }\end{array}$ & $\mathrm{p}$ for interaction \\
\hline LVEF (\%) & $30.9 \pm 10.9$ & $41.3 \pm 12.6$ & $<0.001$ & 0.006 \\
\hline Symptom-guided & $31.6 \pm 10.6$ & $39.0 \pm 11.6$ & $<0.001$ & \\
\hline NT-proBNP-guided & $30.4 \pm 11.1$ & $43.3 \pm 13.0$ & $<0.001$ & \\
\hline LVEDVI (ml/m2) & $87.2 \pm 34.0$ & $70.8 \pm 33.9$ & $<0.001$ & 0.46 \\
\hline Symptom-guided & $85.7 \pm 32.9$ & $70.4 \pm 31.7$ & $<0.001$ & \\
\hline NT-proBNP-guided & $88.4 \pm 35.1$ & $71.2 \pm 35.8$ & $<0.001$ & \\
\hline LVESVI (ml/m2) & $62.2 \pm 30.6$ & $44.3 \pm 30.0$ & $<0.001$ & 0.13 \\
\hline Symptom-guided & $60.7 \pm 28.7$ & $45.2 \pm 27.7$ & $<0.001$ & \\
\hline NT-proBNP-guided & $63.5 \pm 32.3$ & $43.5 \pm 32.0$ & $<0.001$ & \\
\hline LVOT VTI (mm) & $14.9 \pm 5.2$ & $16.8 \pm 5.4$ & 0.009 & 0.37 \\
\hline Symptom-guided & $14.0 \pm 4.5$ & $15.1 \pm 5.6$ & 0.37 & \\
\hline NT-proBNP-guided & $15.5 \pm 5.6$ & $17.9 \pm 5.0$ & 0.007 & \\
\hline $\mathrm{E}(\mathrm{cm} / \mathrm{s})$ & $78 \pm 29$ & $71 \pm 31$ & 0.003 & 0.53 \\
\hline Symptom-guided & $79 \pm 30$ & $71 \pm 33$ & 0.02 & \\
\hline NT-proBNP-guided & $77 \pm 29$ & $72 \pm 29$ & 0.07 & \\
\hline $\mathrm{A}(\mathrm{cm} / \mathrm{s})$ & $64 \pm 29$ & $75 \pm 27$ & $<0.001$ & 0.37 \\
\hline Symptom-guided & $65 \pm 26$ & $74 \pm 22$ & 0.02 & \\
\hline NT-proBNP-guided & $63 \pm 31$ & $76 \pm 30$ & $<0.001$ & \\
\hline$E / A$ & $1.46 \pm 1.14$ & $0.99 \pm 0.67$ & $<0.001$ & 0.87 \\
\hline Symptom-guided & $1.40 \pm 1.09$ & $0.91 \pm 0.56$ & 0.002 & \\
\hline NT-proBNP-guided & $1.51 \pm 1.19$ & $1.06 \pm 0.75$ & 0.002 & \\
\hline $\mathrm{LA}(\mathrm{mm})$ & $46.6 \pm 6.4$ & $44.3 \pm 7.2$ & 0.01 & 0.39 \\
\hline Symptom-guided & $45.7 \pm 5.6$ & $44.8 \pm 7.7$ & 0.27 & \\
\hline NT-proBNP-guided & $45.5 \pm 7.1$ & $43.9 \pm 6.9$ & 0.01 & \\
\hline TAPSE (mm) & $15.7 \pm 4.9$ & $18.6 \pm 5.6$ & $<0.001$ & 0.67 \\
\hline Symptom-guided & $15.6 \pm 4.6$ & $18.6 \pm 4.5$ & $<0.001$ & \\
\hline NT-proBNP-guided & $15.9 \pm 5.2$ & $18.6 \pm 5.7$ & $<0.001$ & \\
\hline dP TR (mmHg) & $32.4 \pm 10.6$ & $30.3 \pm 10.3$ & 0.07 & 0.07 \\
\hline Symptom-guided & $33.9 \pm 9.8$ & $29.5 \pm 9.3$ & 0.01 & \\
\hline NT-proBNP-guided & $31.3 \pm 11.1$ & $30.9 \pm 11.1$ & 0.81 & \\
\hline
\end{tabular}

Data are presented as means $\pm 1 S D$. LVEF=left ventricular ejection fraction. LVEDVI=left ventricular enddiastolic volume. LVESVI=left ventricular end-systolic volume. $P$ for interaction denotes $p$ values for the interaction of NT-proBNP guided treatment with evolution of the individual parameters. 
NT-proBNP-guided heart failure therapy significantly interacted with the changes in LVEF at 18 months, with patients in the symptom-guided group showing a less pronounced increase than patients in the NT-proBNP-guided group ( $p=0.006$ for the effect of NT-proBNP-guided treatment on LVEF over time; Figure 1B). The effect of NTproBNP-guided therapy was also present when patients with only a single plane measurement of LVEF at either baseline or 18 months $(n=24)$ were excluded $(p=0.044$ for the effect of NT-proBNP-guided treatment on LVEF over time). The effect of NT-proBNPguided therapy was present both in patients $60-74$ years old and in patients $\geq 75$ years old ( $p=0.019$ for both groups, Figure $1 C$ and $D)$. The improvement was numerically more pronounced in patients $\geq 75$ years, however, this difference did not reach statistical significance.

A

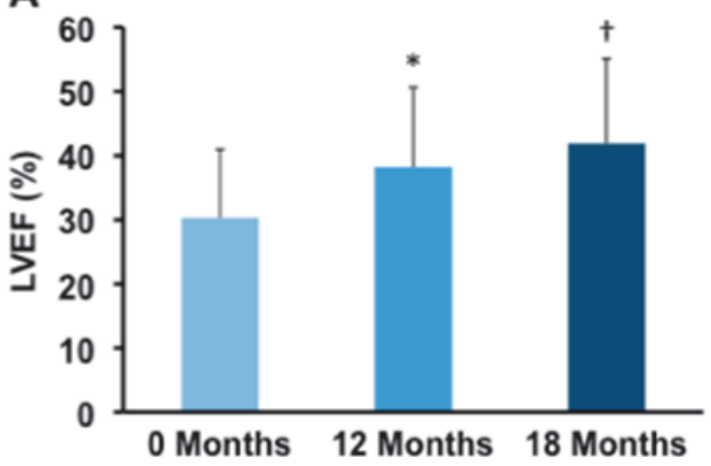

C

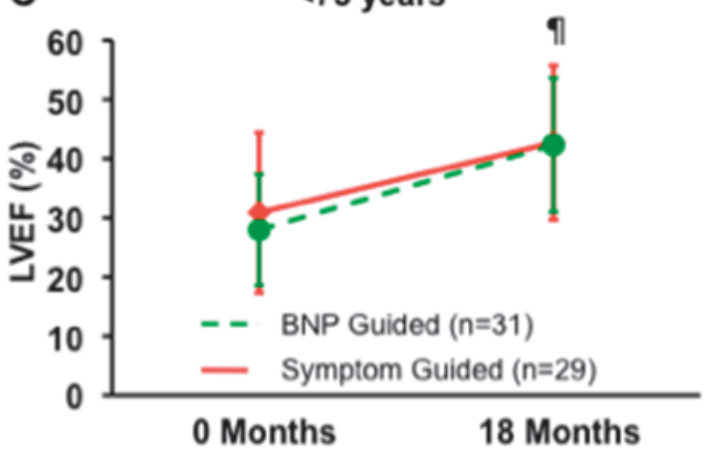

B

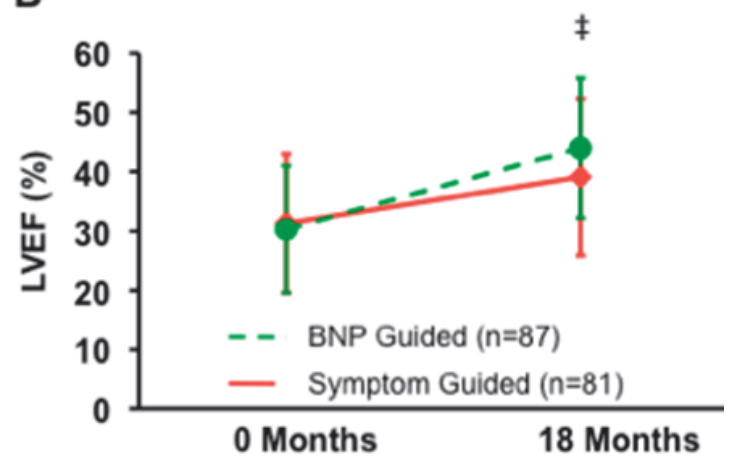

D

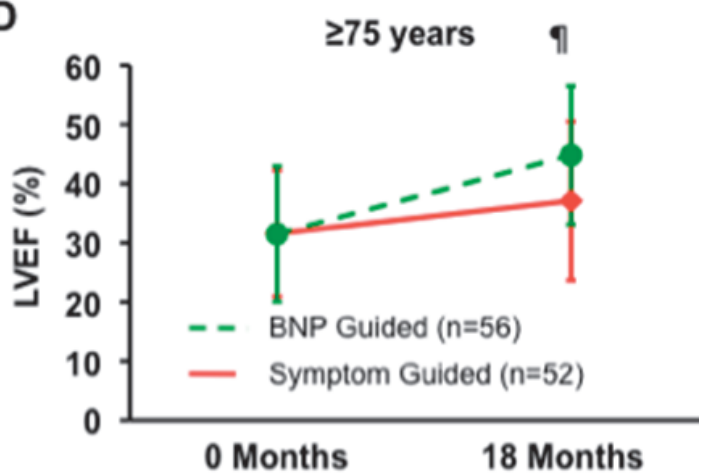

Figure 1. Evolution of LVEF in the study population over time. (A) LVEF in subjects who had evaluable images at 0,12 and 18 months ( $n=168),{ }^{*} p<0.001$ vs baseline, $† p: 0.004$ vs 12 months. (B) LVEF at baseline and 18 months according to assignment to BNP guided or symptom guided treatment strategy, $\ddagger p=0.006$. (C) and (D) evolution of LVEF according to treatment assignment and age. I $p=0.019$

Left ventricular volumes

Reductions in left ventricular volumes occurred throughout the study period in the whole substudy population, with LVEDVI decreasing significantly from 0 to 12 months, and further from 12 to 18 months (Figure $2 \mathrm{~A}, \mathrm{p}<0.001$ for the change from 0 to 12 


\section{CHAPTER 5}

months and from 12 to 18 months). Similarly, LVESVI decreased significantly from 0 to 12 months and further from 12 to 18 months (Figure 2A, $p<0.001$ for the change from 0 to 12 months and from 12 to 18 months). The changes in LVEDVI and LVESVI were not influenced age or by NT-proBNP-guided therapy (Figure $2 \mathrm{~B}$ and $\mathrm{C} ; \mathrm{p}=0.396$ for the effect of NT-proBNP-guided therapy on the change in LVESVI at 18 months, $p=0.853$ for the effect of NT-proBNP-guided therapy on the change in LVEDVI at 18 months).

A
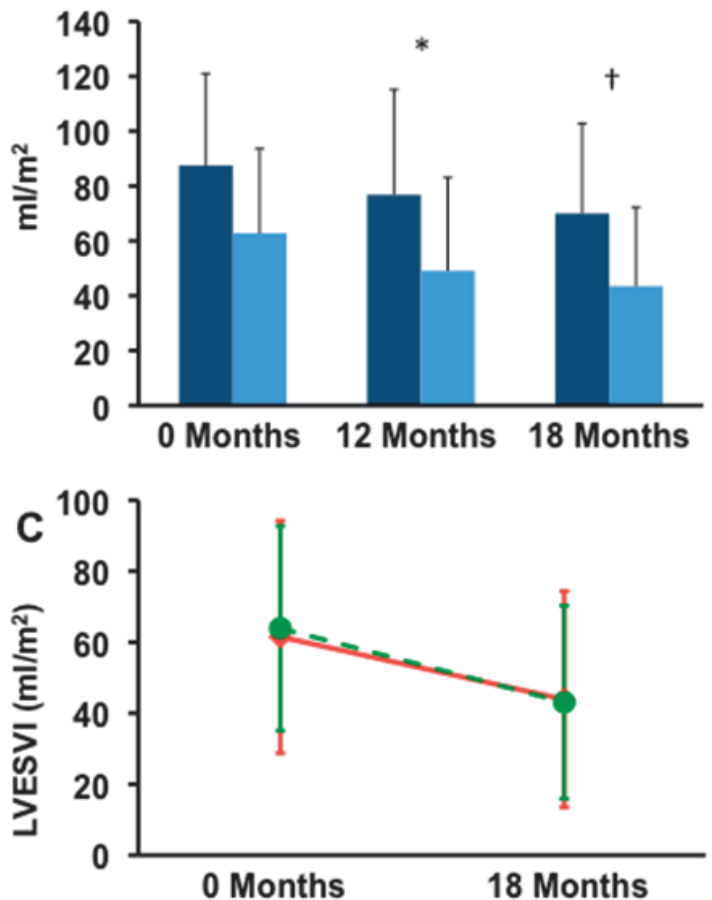

B

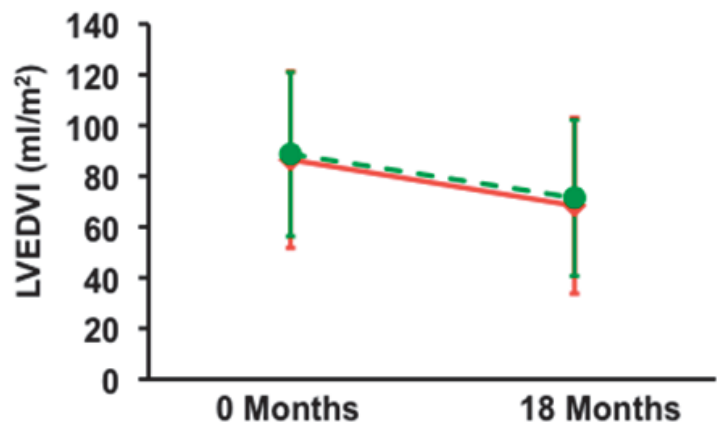

Figure 2. Left ventricular remodeling in the study population over time. (A) LVEDVI and LVESVI in subjects who had evaluable images at 0,12 and 18 months ( $n=168$ ), * $p<0.001$ vs 0 months, $\dagger p<0.001$ vs 12 months. (B) LVEDI at baseline and 18 months according to assignment toNT-proBNP guided or symptom-guided treatment strategy. $p$ for interaction 0.853. (C) LVESVI at 0 and 18 months according to treatment assignment. $p$ for interaction 0.396

Left atrial size, diastolic left ventricular function, and right ventricular function

Additional echocardiographic data were analyzed in 188 patients (table 2). Overall, there was no significant interaction between NT-proBNP-guided treatment and changes in these additional parameters. Compared to baseline, the velocity time integral, and thus flow, in the left ventricular outflow tract increased significantly in patients on NTproBNP-guided therapy but not in those on symptom-guided therapy. Left atrial size decreased over time, this decrease tended to be larger in patients with NT-proBNPguided therapy. Likewise, the E/A ratio decreased significantly in both patient groups. 
TAPSE increased in both groups, while the right ventricular-right atrial pressure gradient decreased significantly only in patients on symptom-guided therapy.

\section{Effect of changes in Left Ventricular Ejection Fraction on Outcome}

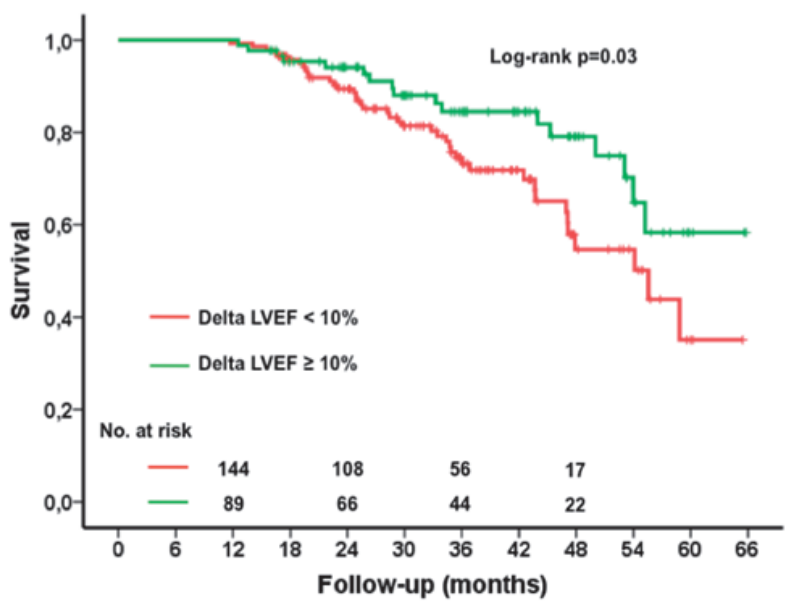

Figure 3. Survival in patients with $<10 \%$ vs. $10 \%$ improvement in LVEF.

The present TIME-CHF echocardiographic substudy was underpowered to detect an effect of NT-proBNP-guided therapy on outcome according to echocardiographic parameters. However, to obtain some measure of the effect of changes in LVEF on therapy on outcome, 233 patients with complete echocardiography datasets at 0 and 12 months were divided into those with an absolute increase in LVEF of $\geq 10 \%$ at 12 months versus those with a smaller increase or a decrease in LVEF. Kaplan Meier curves showed a significantly better 18-month survival in patients with an increase in LVEF $\geq 10 \%$ compared to those with less change in LVEF ( $\log$ rank $p=0.030$; Figure 3 ). Of note, the proportion of patients experiencing an increase in LVEF $\geq 10 \%$ did not differ between the age groups or between treatment strategies (data not shown). Also, baseline NT-proBNP did not differ between patients experiencing an increase in LVEF $\geq 10 \%$ versus those experiencing an increase $<10 \%$ (median 3891 (interquartile range 1575-6207) $\mathrm{pg} / \mathrm{ml}$ versus 3563 (1466-5680) $\mathrm{pg} / \mathrm{ml}, \mathrm{p}=0.79)$.

\section{DISCUSSION}

In this substudy of TIME-CHF, intensified heart failure treatment led to reverse remodeling that continued up to 18 months after enrolment. In particular, intensified NTproBNP-guided treatment significantly interacted with the increase in LVEF independent of age and the duration of heart failure. Changes in left ventricular function had an 
influence on survival, as patients with an absolute increase in LVEF by 10 or more percentage points at one year experienced a better survival than patients without.

While the prognostic value of LVEF in populations including heart failure patients with preserved ejection fraction has been questioned ${ }^{18}$, the extent of reverse remodeling expressed either as improvement in LVEF or decrease in left ventricular volumes has been associated with improved prognosis in patients with reduced LVEF irrespective of heart failure etiology ${ }^{19,20}$. Interestingly, the degree of ventricular remodeling seen in the present substudy of TIME-CHF by intensifying standard heart failure medication was substantial and comparable to the improvements found with resynchronization therapy ${ }^{5}$. Changes in LVEF reported for drug interventions with angiotensin converting enzyme inhibitors, angiotensin receptor blockers and betablockers range from a neutral effect in the SOLVD echocardiography substudy ${ }^{20}$ to an increase by app. 5 percentage points in the Val-HeFT echocardiographic substudy and the MOCHA study ${ }^{21,}{ }^{22}$. When comparing our results with these studies, it should be noted that we analyzed only patients with available echocardiograms at all time-points (baseline, 12 and 18 months). In contrast, in the aforementioned trials baseline echocardiograms of patients who later died or dropped out of the study and did not have follow-up echocardiography were also included in the analyses. Thus, the respective effects of the therapeutic intervention on left ventricular remodeling may even have been overestimated due to lacking follow-up in patients with a low LVEF at study inclusion who died. Moreover, most patients in the present study were on standard heart failure medication already at baseline and improvement in LVEF was also seen in those with long-standing heart failure. Further, the mean age of patients included in the mentioned trials ranged from 60 to 65 years and was considerably lower than in TIME-CHF. Our data, therefore, question the notion that reverse remodeling by medical therapy is limited or even absent in chronic heart failure and highlight that reverse remodeling can be also achieved with intensified heart failure therapy. This may be even achieved in elderly, which represent the majority of heart failure patients. Importantly, reverse remodeling was independent of the prior duration of heart failure, implying that even in advanced heart failure, uptitration of heart failure therapy may be of benefit.

Increased natriuretic peptides are powerful predictors of outcome in heart failure. As a consequence, several studies have examined the value of NT-proBNP-guided therapy on outcome. Since the first small trial suggesting a benefit ${ }^{23}$, several though not all studies have found a benefit of NT-proBNP-guided therapy on outcome, which, however, seems to be restricted to patients $<75$ years of age ${ }^{11,24}$, as first described in TIME$\mathrm{CHF}$, which included a large number of patients $\geq 75$ years. In contrast to the findings on the main outcome measures in TIME-CHF but also other studies ${ }^{24}$ and a recent metaanalysis ${ }^{25}$, NT-proBNP-guided heart failure treatment in that patient group significantly interacted with the increase in LVEF similar to that observed in younger patients. We therefore speculate that the neutral results of NT-proBNP-guided treatment strategies in the elderly do not relate to a lack of effectiveness of intensified heart failure treat- 
ment, but rather to the dilution of the effect by the large number of comorbidities which significantly impacted on outcome but were not influenced by intensifying heart failure therapy. In that aspect, the GUIDing Evidence based therapy using biomarker Intensified Treatment (GUIDE-IT) trial will include a larger patient population and thus will be able to further define the value of biomarker guided therapy in elderly patients with regards to clinical endpoints.

Overall, our results are in line with the results from the PROTECT trial that had shown reverse remodeling both in patients on symptom-guided and on NT-proBNPguided therapy, with a larger effect in the latter. However, there are important differences between the two studies that should be noted. Our study population was on average 13 years older than in PROTECT, which significantly adds to our understanding, given the differences found in clinical outcome depending on age ${ }^{25}$. Also, TIME-CHF was a multicenter trial that recruited patients from smaller, non-tertiary care centers too. Thus, the patient population included in TIME-CHF is likely closer to a real world heart failure population. In addition, our patients had higher NT-proBNP values at inclusion (median NT-proBNP in PROTECT $2118 \mathrm{pg} / \mathrm{ml}$ vs. $3832 \mathrm{pg} / \mathrm{ml}$ in our study), and no patients with a biventricular pacemaker were included in the present study vs. $40 \%$ of all patients in PROTECT. Thus, our results confirm the results of PROTECT, but extend the findings to a broader and more real-world heart failure patient population. Additional echocardiographic parameters showed a reduction in left atrial size, improvement in diastolic and right ventricular function and a reduction in pulmonary pressure. In contrast to PROTECT, these changes did not depend on NT-proBNP-guided therapy. Nevertheless, these data are important, because they add further support to the notion that intensified heart failure therapy is able to reverse the functional consequences of heart failure with reduced ejection fraction.

We conducted additional analyses exploring the association in improvement in left ventricular ejection fraction with outcome. The selection of a cut-off of 10 percent improvement in ejection fraction was based on previous data indicating 10 percentage points as the upper limit of temporal variability of two-dimensional measurements of ejection fraction ${ }^{17}$, and thus changes $\geq 10 \%$ can be assumed to represent true improvements in individual patients. While the survival analyses presented here are of exploratory character and do not take into account treatment strategy assignment, they suggest that improvements in left ventricular ejection fraction did translate into advantages in prognosis.

There are limitations to this study. The TIME-CHF trial was designed to compare two strategies for treatment of heart failure in patients over the age of 60 years. While the two subgroups were defined a priori, the age groups were not large enough to investigate the influence of left ventricular remodeling on outcome according to age group, which would have been of particular interest in patients $\geq 75$ years. Evaluable echocardiograms were available only in two thirds of eligible patients. However, this proportion is similar to values reported from other heart failure trials ${ }^{6,21}$, and the baseline charac- 


\section{CHAPTER 5}

teristics of patients included in the present substudy did not differ from the total patient population.

In summary, we show that clinically significant reverse remodeling occurs with intensified medical therapy in heart failure patients independent of age, including a significant number of patients aged $\geq 75$ years. NT-proBNP-guided treatment significantly interacted with the improvement in LVEF in both age groups suggesting that larger increase in heart failure therapy is associated with a greater change in LV function, reverse remodelling and as a consequence better outcome than standard therapy, independent of age. 


\section{IMPROVEMENT IN LEFT VENTRICULAR EJECTION FRACTION AND ...}

\section{REFERENCES}

1. Solomon SD, Anavekar N, Skali $\mathrm{H}$ et al. Influence of ejection fraction on cardiovascular outcomes in a broad spectrum of heart failure patients. Circulation. 2005;112:3738-3744

2. White HD, Norris RM, Brown MA, Brandt PW, Whitlock RM, Wild CJ. Left ventricular end-systolic volume as the major determinant of survival after recovery from myocardial infarction. Circulation. 1987;76:44-51

3. Greenberg B, Quinones MA, Koilpillai C et al. Effects of long-term enalapril therapy on cardiac structure and function in patients with left ventricular dysfunction. Results of the solvd echocardiography substudy. Circulation. 1995;91:2573-2581

4. Doughty RN, Whalley GA, Gamble G, MacMahon S, Sharpe N. Left ventricular remodeling with carvedilol in patients with congestive heart failure due to ischemic heart disease. Australia-new zealand heart failure research collaborative group. J Am Coll Cardiol. 1997;29:1060-1066

5. St John Sutton M, Ghio S, Plappert T et al. Cardiac resynchronization induces major structural and functional reverse remodeling in patients with new york heart association class i/ii heart failure. Circulation. 2009;120:1858-1865

6. Tardif JC, O'Meara E, Komajda M et al. Effects of selective heart rate reduction with ivabradine on left ventricular remodelling and function: Results from the shift echocardiography substudy. Eur Heart J. 2011;32:2507-2515

7. Ezekowitz JA, McAlister FA. Aldosterone blockade and left ventricular dysfunction: A systematic review of randomized clinical trials. Eur Heart J. 2009;30:469-477

8. Weir RA, Mark PB, Petrie CJ et al. Left ventricular remodeling after acute myocardial infarction: Does eplerenone have an effect? Am Heart J. 2009;157:1088-1096

9. Cleland JG, Swedberg K, Follath F et al. The euroheart failure survey programme-- a survey on the quality of care among patients with heart failure in europe. Part 1: Patient characteristics and diagnosis. Eur Heart J. 2003;24:442-463

10. Roger VL, Go AS, Lloyd-Jones DM et al. Heart disease and stroke statistics--2012 update: A report from the american heart association. Circulation. 2012;125:e2-e220

11. Januzzi JL, Jr., Rehman SU, Mohammed AA et al. Use of amino-terminal pro-b-type natriuretic peptide to guide outpatient therapy of patients with chronic left ventricular systolic dysfunction. J Am Coll Cardiol. 2011;58:1881-1889

12. Weiner RB, Baggish AL, Chen-Tournoux A et al. Improvement in structural and functional echocardiographic parameters during chronic heart failure therapy guided by natriuretic peptides: Mechanistic insights from the probnp outpatient tailored chronic heart failure (protect) study. Eur J Heart Fail. 2013;15:342-351

13. Pfisterer $\mathrm{M}$, Buser $\mathrm{P}$, Rickli $\mathrm{H}$ et al. Bnp-guided vs symptom-guided heart failure therapy: The trial of intensified vs standard medical therapy in elderly patients with congestive heart failure (time-chf) randomized trial. JAMA. 2009;301:383-392

14. Brunner-La Rocca HP, Buser PT, Schindler R et al. Management of elderly patients with congestive heart failure--design of the trial of intensified versus standard medical therapy in elderly patients with congestive heart failure (time-chf). Am Heart J. 2006;151:949-955

15. Lang RM, Bierig M, Devereux RB et al. Recommendations for chamber quantification: A report from the american society of echocardiography's guidelines and standards committee and the chamber quantification writing group, developed in conjunction with the european association of echocardiography, a branch of the european society of cardiology. J Am Soc Echocardiogr. 2005;18:1440-1463

16. Rudski LG, Lai WW, Afilalo J et al. Guidelines for the echocardiographic assessment of the right heart in adults: A report from the american society of echocardiography endorsed by the european association of echocardiography, a registered branch of the european society of cardiology, and the canadian society of echocardiography. J Am Soc Echocardiogr. 2010;23:685-713; quiz 786-688 


\section{CHAPTER 5}

17. Thavendiranathan P, Grant AD, Negishi T, Plana JC, Popovic ZB, Marwick TH. Reproducibility of echocardiographic techniques for sequential assessment of left ventricular ejection fraction and volumes: Application to patients undergoing cancer chemotherapy. J Am Coll Cardiol. 2013;61:77-84

18. van Veldhuisen DJ, Linssen GC, Jaarsma T et al. B-type natriuretic peptide and prognosis in heart failure patients with preserved and reduced ejection fraction. J Am Coll Cardiol. 2013;61:1498-1506

19. Di Biase L, Auricchio A, Sorgente A et al. The magnitude of reverse remodelling irrespective of aetiology predicts outcome of heart failure patients treated with cardiac resynchronization therapy. Eur Heart J. 2008;29:2497-2505

20. Hoshikawa E, Matsumura Y, Kubo T et al. Effect of left ventricular reverse remodeling on long-term prognosis after therapy with angiotensin-converting enzyme inhibitors or angiotensin ii receptor blockers and beta blockers in patients with idiopathic dilated cardiomyopathy. Am J Cardiol. 2011;107:1065-1070

21. Wong $M$, Staszewsky L, Latini $R$ et al. Valsartan benefits left ventricular structure and function in heart failure: Val-heft echocardiographic study. J Am Coll Cardiol. 2002;40:970-975

22. Bristow MR, Gilbert EM, Abraham WT et al. Carvedilol produces dose-related improvements in left ventricular function and survival in subjects with chronic heart failure. Mocha investigators. Circulation. 1996;94:2807-2816

23. Troughton RW, Frampton CM, Yandle TG, Espiner EA, Nicholls MG, Richards AM. Treatment of heart failure guided by plasma aminoterminal brain natriuretic peptide ( $\mathrm{n}$-bnp) concentrations. Lancet. 2000;355:1126-1130

24. Lainchbury JG, Troughton RW, Strangman KM et al. N-terminal pro-b-type natriuretic peptide-guided treatment for chronic heart failure: Results from the battlescarred (nt-probnp-assisted treatment to lessen serial cardiac readmissions and death) trial. J Am Coll Cardiol. 2009;55:53-60

25. Troughton RW, Frampton CM, Brunner-La Rocca HP et al. Effect of b-type natriuretic peptide-guided treatment of chronic heart failure on total mortality and hospitalization: An individual patient metaanalysis. Eur Heart J. 2014 


\section{CHAPTER 6}
Altered left ventricular geometry and
torsional mechanics in high altitude-induced pulmonary hypertension:
a 3D echocardiographic study

B.W. De Boeck MD, S. Kiencke MD, C. Dehnert MD, Stefanie Zügel MS, K. Auinger MD, M. Maggiorini MD, B.A. Kaufmann MD

Under Review 


\section{CHAPTER 6}

\section{ABSTRACT}

Introduction: Changes in left ventricular (LV) torsion have been related to LV geometry in patients with concomitant long-standing myocardial disease or pulmonary hypertension $(\mathrm{PH})$. We evaluated the effect of acute high altitude-induced PH on LV geometry, volumes, systolic function and torsional mechanics.

Methods: 23 volunteers were studied at low altitude and after the second ("D3") and third night ("D4") at high altitude $(4559 \mathrm{~m})$. LV ejection fraction, multidirectional strains and torsion, LV volumes, sphericity, and eccentricity were derived by speckle tracking on 3D echocardiographic datasets. Pulmonary pressure was estimated from the transtricuspid pressure gradient (TRPG), LV preload from enddiastolic LV volume and transmitral over mitral annular E velocity (E/e').

Results: At high altitude, oxygen saturation decreased by 15-20\%, heart rate and cardiac index increased by $15-20 \%$, and TRPG increased from $21 \pm 2$ to $37 \pm 9 \mathrm{mmHg}(p<0.01)$. LV volumes, preload, ejection fraction, multidirectional strains and sphericity remained unaffected, but diastolic $(1.04 \pm 0.07$ to $1.09 \pm 0.09$ on D3/D4, $p<0.05)$ and systolic $(1.00 \pm 0.06$ to $1.08 \pm 0.1$ (D3) and $1.06 \pm 0.07$ (D4), $p<0.05)$ eccentricity slightly increased, indicating mild LV D-shaping. LV torsion decreased from $2.14 \pm 0.85$ to $1.34 \pm 0.68$ $(p<0.05)$ and $1.65 \pm 0.54(p=0.08)$ degrees/cm on D3/D4, respectively. Changes in torsion were weakly but significantly related to changes in systolic $(r=-0.369, p=0.013)$ and diastolic ( $r=-0.329, p=0.032$ ) eccentricity, but not to changes in TRPG, heart rate or preload.

Conclusion: High altitude exposure was associated with mild D-shaping of the ventricle and reduced ventricular torsion at unchanged global left ventricular function and preload, suggesting a relation between LV geometry and torsional mechanics. 


\section{INTRODUCTION}

The left ventricle reshapes during the cardiac cycle in order to reduce the volume of its cavity against the load imposed on it. Besides myocardial shortening and thickening, this deformation encompasses a characteristic wringing motion of the left ventricle along its longitudinal axis, referred to as left ventricular (LV) twist or torsion ${ }^{1}$. LV torsion is characterized by a small clockwise rotation of the base, and a larger counterclockwise in the apical region, attributed to a dynamic interaction between shortening in nearly perpendicularly oriented myofibre helices in the subendocardial and subepicardial layers ${ }^{1}$. By deforming the subendocardial fibre matrix in the cross-fibre direction (cross-fibre shortening), torsion during ejection in turn is believed to act as a systolic amplification mechanism, contributing to the transformation of a $15-20 \%$ shortening of the active contractile elements (myofibres) into a volume reduction of about $60 \%$ at the left ventricular cavity level ${ }^{2}$. Consistent with the dependence on both myofibre shortening as well as myofibre orientation across and along the LV myocardium, a number of studies have not only identified myocardial loading and contractility, but also concentric remodelling to influence torsion in the human heart. Studies reporting on the specific role of LV geometry have been scarce and generally limited to patient populations with longstanding myocardial disease, remodelling and LV loading as potential confounders ${ }^{3-7}$. We sought to assess the influence of short-term cardiac shape changes and pulmonary hypertension ( $\mathrm{PH}$ ) on LV torsion in healthy volunteers rapidly ascending to high altitude. Given the three-dimensional (3D) nature of LV geometry and deformation as well as the advantage of 3D echocardiography with regard to plane alignment standardization, 3D speckle tracking echocardiography was used to calculate LV volumes, geometry, torsion and multidirectional deformation (strains).

\section{METHODS}

\section{Study participants}

The study recruited 25 healthy volunteers between 18 and 65 years for a multidisciplinary high altitude physiology study. Criteria of exclusion included chronic intake of medication, an abnormal baseline echocardiogram (mild valvular regurgitation was accepted), or known cardiopulmonary and other chronic diseases. Furthermore, we excluded volunteers who had spent more than 5 nights at an altitude higher than $2500 \mathrm{~m}$ within the last 30 days. The study conformed to the Declaration of Helsinki and was approved by the Ethical Committee of the University of Zurich. All subjects gave written informed consent to participation. Two subjects were excluded from analysis because of poor 3D echocardiographic windows. 


\section{CHAPTER 6}

\section{Study Protocol}

Three to four weeks after baseline measurements at the University Hospital of Zurich ("Base", 450 m), study participants travelled to Alagna (Italy, $1205 \mathrm{~m}$ ) from where they were carried by cable car to an altitude of $2900 \mathrm{~m}$. They continued on foot to the Gnifetti hut $(3647 \mathrm{~m})$ where they spend the night. After an ascent of 4 to 6 h they reached the Capanna Regina Margherita research facility (Italy, $4559 \mathrm{~m}$ ) around noon of the following day (day 1). Cardiopulmonary testing including standard and 3D echocardiography was performed on the morning following the first ("D2"), second ("D3") and third night ("D4") spent at the Margherita hut. Peripheral arterial O2-saturation (SatO2) was measured by pulse oximetry, Blood pressure was measured immediately prior to echocardiography, resting heart rate during the 3D-acquisition was derived from the 3lead electrocardiogram of the ultrasound machine.

\section{Echocardiography}

Resting transthoracic echocardiography was performed on a high-end echocardiographic system (Aplio Artida, Toshiba Medical Systems Corp., Tokyo, Japan) equipped with a phased array 2D (PST-30SBT) and a matrix array 3D (PST-25SX) transducer. Pulsed-, continuous-wave and colour-coded Doppler, and 2D-acquisitions were performed at end-expiratory breath-hold and measurements averaged over 2-4 beats. For the 3Dbased analysis, wide-angled $\left(80^{\circ}\right.$ by $\left.80^{\circ}\right)$ 'full volume' mode was used, in which four to six wedge-shaped sub-volumes were acquired over four to six consecutive cardiac cycles during a single breath-hold, resulting in a pyramidal 4D dataset at 17 to 25 frames (mean 18.7+1.2) per second ${ }^{8,9}$.

\section{Doppler and 2D based echocardiographic analysis}

From the mitral-inflow pattern, the peak of the early (E) and the effective late (Aeff) filling velocities and their ratio were determined, with Aeff calculated as $A-E$ at $A$, with $E$ at $A$ representing the residual $E$ velocity at onset of the A-wave in case of partial fusion. The tissue Doppler early systolic (LV-s') and diastolic ( $\left.e^{\prime}\right)$ velocity registered at the inferoseptal and lateral mitral annulus was averaged; the ratio $\mathrm{E} / \mathrm{e}^{\prime}$ was considered to reflect left ventricular filling pressure ${ }^{10}$. Right atrial pressure was estimated from the inferior vena cava size and respiratory collapsibility ${ }^{11,12}$. Systolic (RVPGs) and diastolic (RVPGd) right ventricular pressure gradients were derived from the modified Bernoulli equation on the continuous-wave Doppler traces of the transtricuspid and transpulmonary regurgitant jets, respectively ${ }^{11,12}$. For the assessment of RV function and size, RV end-diastolic (RV-EDA), and end-systolic area (RV-ESA), the resulting fractional area change (RV-FAC), the tissue Doppler-derived systolic velocity (RV-s') and M-mode de- 
rived tricuspid annular plane systolic excursion (TAPSE) were measured on apical fourchamber views ${ }^{12}$.

\section{Three-dimensional speckle tracking deformation analysis}

Pyramidal 3D datasets were analysed using 3D wall motion tracking software (Toshiba Medical Systems) by a single, experienced investigator. The 3-D dataset was aligned along the LV maximal long axis crossing the true apex and the mitral annular plane. Then, a vertical long axis (VLA) image plane parallel to the interventricular septum (IVS), a horizontal long axis (HLA) image plane perpendicular to the VLA plane and bisecting the IVS, and 3 equally distributed short-axis sections were reconstructed (Figure 1$)^{13}$. After defining an endocardial region of interest adjusted to include trabeculae and papillary muscles in the LV, the endocardial surface and epicardial bounderies were defined semi-automatically. Finally, automatic speckle tracking of the resulting 3D region of interest was performed and fine-tuned using the cine-loop play feature as previously described in detail ${ }^{8,9}$. For the purpose of the current study, global torsion was analyzed and compared to global LV strains and geometry. LV mass, enddiastolic (LVEDV) and endsystolic (LVESV) as well as stroke (LVSV) volumes were derived from the volumetric data, respectively.

\section{D speckle tracking-based LV geometry}

LV-sphericity was defined as the actual 3D LV volume, divided by the volume of a sphere with the LV major long axis length as diameter ${ }^{14}$. To express septal flattening (Dshaping) on the global LV level, LV eccentricity was defined as (area VLA/area HLA). Local eccentricity was calculated from the basal, mid-ventricular, and apical short axis views as D-VLA/D-HLA, where D-VLA and D-HLA represent the local diameters in the VLA and HLA planes, respectively (Figure 1) ${ }^{12,15}$. All measures were performed at enddiastole and end-systole using the speckle tracking-derived endocardial boundaries on the axis-corrected views. 


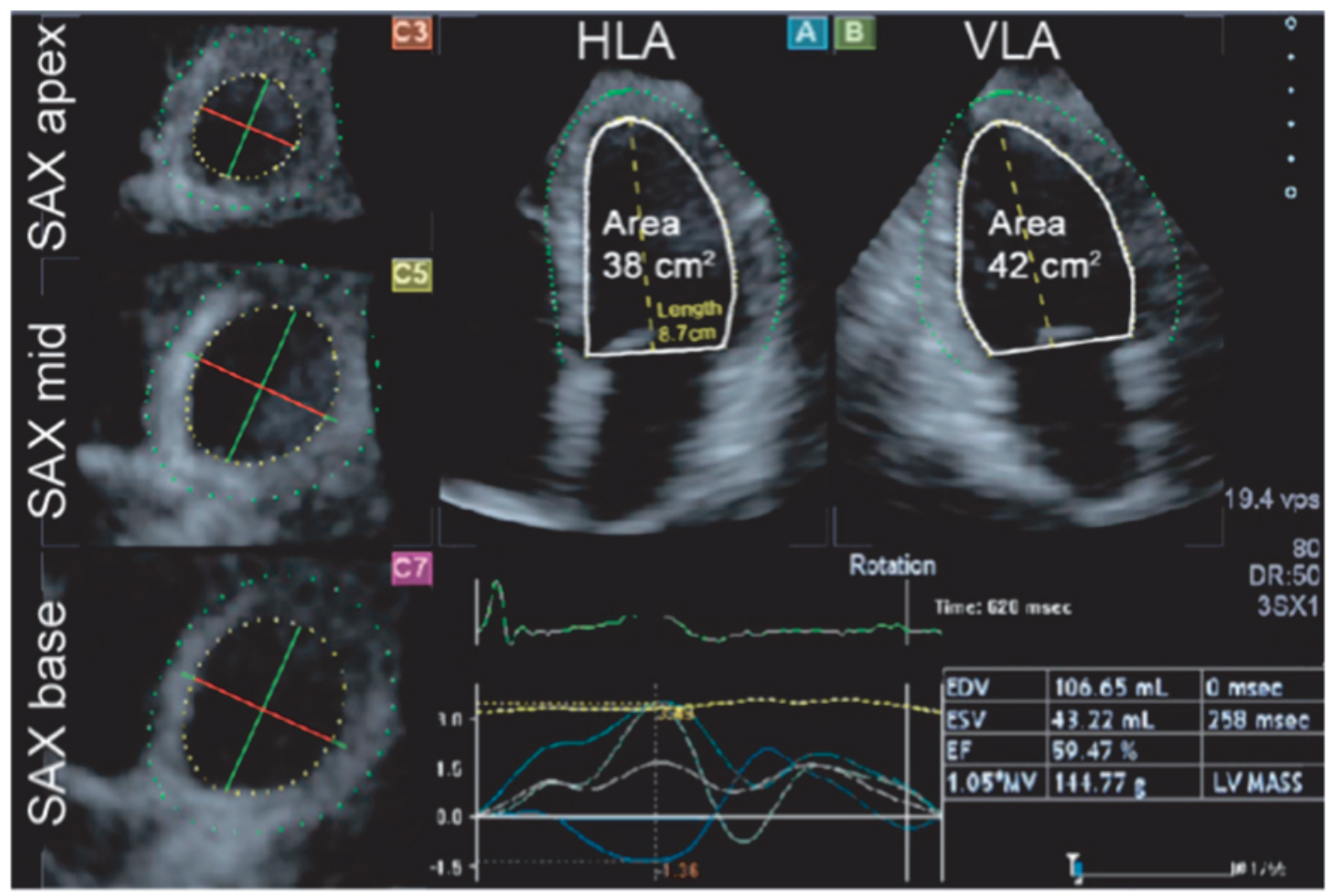

Figure 1. 3D plane alignment and geometry measurements on an end-diastolic frame. Top middle and right panels show the HLA and VLA planes aligned along the LV longitudinal axis, left panels the true short axis images perpendicular to the latter (see text for details). The green and red section lines on the true short axis images correspond to the VLA and HLA planes and determine the local D-VLA and D-HLA length at each level, respectively. For clarity, D-VLA (in green) is projected under the D-HLA direction (in red). Note that LV eccentricity (area VLA/area HLA) as well as local eccentricity (D-VLA/D-HLA) at the base and midventricular level is $>1$.

\section{Reproducibility}

In a random sample of 20 studies, 3D speckle tracking was repeated at least 4 months after the initial analysis by the same observer, blinded to the results of prior measurements. The coefficient of variation ranged from 7.5\% (LV mass and LV-EDV) to $14 \%$ (LVESV) for 3D-derived volumes, from 6.5\% (area strain) to $16.7 \%$ (radial strain) for global LV strain measurements and was $24 \%$ for LV torsion. Systematic bias expressed as a percentage of the initial measurement, was $\leq 3.5 \%$ for all, except for radial strain (8.4\%) For LV diastolic / systolic eccentricity the coefficient of variation was 7.1\% / 9.7\% with a bias $\leq 2.0 \%$.

\section{Statistical analysis}

Continuous variables are presented as mean $\pm S D$, categorical data as numbers and percentages. Because of a low number of technically adequate 3D acquisitions on D2 due to more pronounced tachycardia, invalidating mountain sickness and resting dyspnoea, 
D2 measurements were excluded from the analysis. A repeated measures analysis of variance with Sidak-Bonferroni post hoc testing to correct for the inflation of type 1 error was used to test for significant differences in hemodynamics, LV geometry, deformation and function measures between the 3 time points. When systolic and diastolic parameters were compared at a particular stage, a paired T-test was applied. The relation between changes in LV torsion, in hemodynamics, RV/LV functional parameters and LV geometry was assessed using linear regression analysis. Statistical analyses were performed with SPSS version 20.0 (SPSS, Inc., Chicago, IL). A p value $<.05$ was considered statistically significant.

\section{RESULTS}

The final study population comprised 23 healthy (age $43 \pm 9$ years, 8 female, BSA $1.73 \pm 0.16 \mathrm{~m} 2, \mathrm{BMI} 24 \pm 2 \mathrm{~kg} / \mathrm{m} 2$ ) volunteers. Resting hemodynamic data and changes induced by altitude are displayed in Figure 2. Exposure to altitude was associated with a drop in $\mathrm{SpO} 2$ of about $15-20 \%$, which appeared to be largely compensated for by a rise in cardiac output of 15-20\% (Figure 2A\&B). Most of this increased cardiac output at rest could in turn be ascribed to a comparable rise in resting heart rate, implying that stroke volume did not change. Exposure to high altitude markedly augmented systolic and diastolic RVPG (RVPGs/RVPGd), from a mean of 21/4 mmHg at baseline to a mean of $38 / 13$ and 36/12 mmHg at D3 and D4, respectively (Figure 2C). Systemic blood pressures remained unchanged throughout all stages (Figure 2D). 

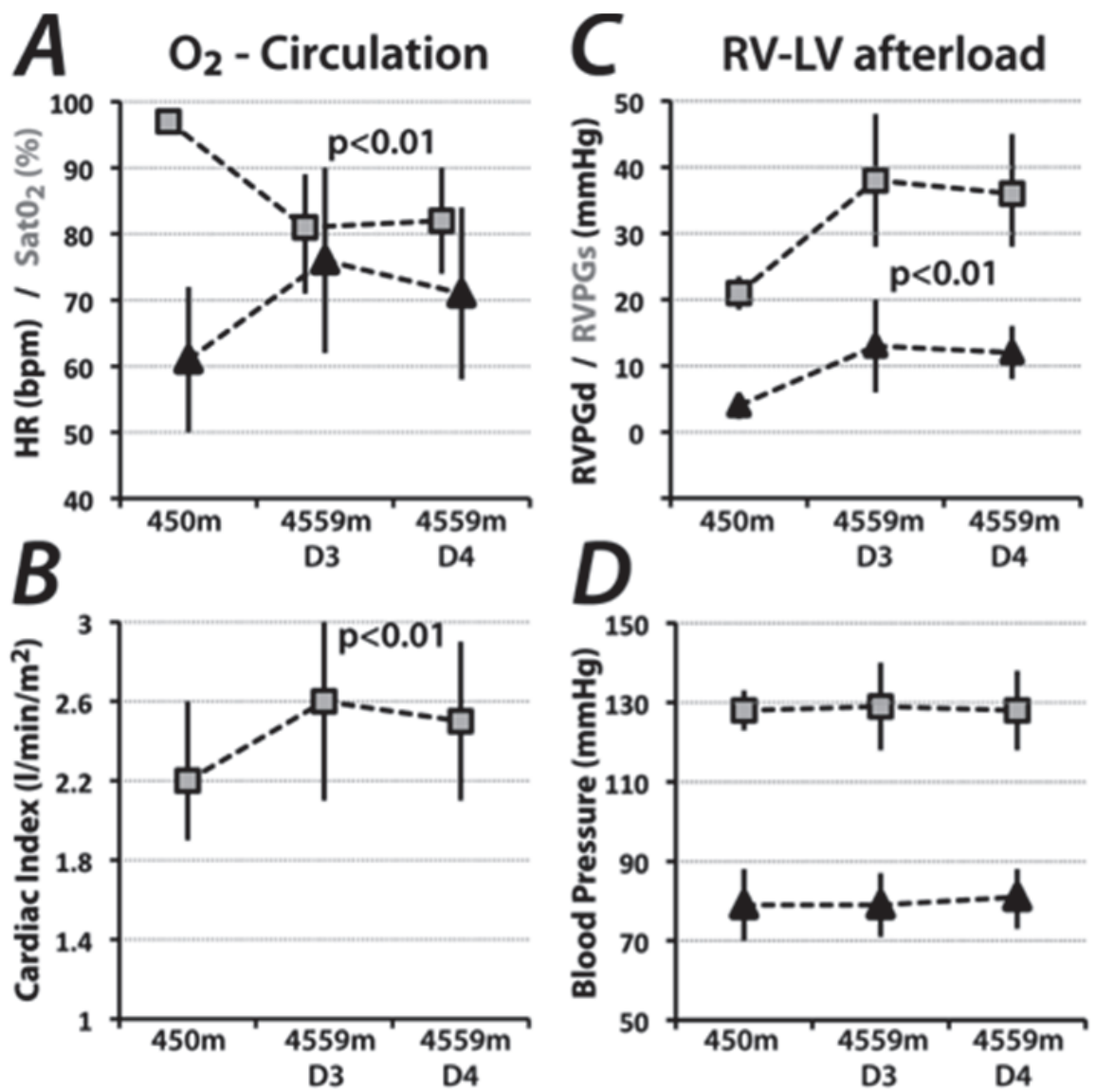

Figure 2. Hemodynamics and ventricular loading at baseline and attitude. HR (bpm): heart rate in beats/minute, all other abbreviations as explained in the main document (methods). The p-values indicated in each graph refer to the significance level for both D3 and D4 compared to baseline, and for each parameter (on y-axis) within the graph. RVPGd/RVPGs and blood pressure are regarded as markers of RV and LV afterload, respectively.

\section{LV and RV function by echocardiography}

Echocardiographic characteristics at baseline and at altitude are shown in Table 1. Compared to the baseline measurements, LV torsion was impaired at altitude. After correction for multiple comparisons statistical significance was retained at D3, with a strong trend at D4 ( $p=0.08$ versus baseline). Conversely, systolic annular velocities significantly increased. Other than in torsion and systolic annular velocities, no changes were noticed between baseline and altitude at the LV side, whether considering parameters reflecting overall LV pumping and diastolic function, the individual strain components, or the volumes needed to maintain this systolic function. On the RV side, altitude exposure was associated with increases in RV-EDA and RV-ESA, which reached significance at D3. Except for RV-s' at D4, RV systolic function parameters at altitude did not significantly differ from the baseline values.. 
Table 1. RV and LV echocardiograpic data at baseline and altitude

\begin{tabular}{llll}
\hline & $450 \mathrm{~m}$ & $4559 \mathrm{~m} \mathrm{D} 3$ & $4559 \mathrm{~m} \mathrm{D4}$ \\
\hline RV EDA $\left(\mathrm{cm}^{2}\right)$ & $20 \pm 4.6$ & $22 \pm 4.3+$ & $22 \pm 4.6$ \\
RV ESA $\left(\mathrm{cm}^{2}\right)$ & $11 \pm 3.0$ & $13 \pm 3.0 \#$ & $12 \pm 2.9$ \\
RV FAC (\%) & $46 \pm 9.9$ & $42 \pm 8.3$ & $45 \pm 9.0$ \\
RV-s' & $15.7 \pm 0.7$ & $16.7 \pm 0.8$ & $18.5 \pm 0.8+*$ \\
TAPSE (mm) & $26 \pm 5$ & $25 \pm 3$ & $27 \pm 5 *$ \\
LV mass (mg/ml) & $154 \pm 33$ & $153 \pm 33$ & $155 \pm 30$ \\
LV EDV (ml) & $117 \pm 22$ & $114 \pm 27$ & $118 \pm 22$ \\
LV ESV (ml) & $50 \pm 11$ & $50 \pm 15$ & $49 \pm 12$ \\
LV SV (ml) & $67 \pm 13$ & $64 \pm 13$ & $69 \pm 13 *$ \\
LV EF (\%) & $57 \pm 4.6$ & $57 \pm 4.3$ & $59 \pm 5.2$ \\
LV-s' & $9.7 \pm 1.7$ & $11.5 \pm 2.0+$ & $12.3 \pm 2.0 \#$ \\
E/A eff & $1.4 \pm 0.3$ & $1.5 \pm 0.6$ & $1.6 \pm 0.6$ \\
e' & $10.7 \pm 0.5$ & $10.8 \pm 0.6$ & $13.3 \pm 1.2$ \\
E / e' & $6.9 \pm 1.5$ & $6.7 \pm 1.8$ & $6.7 \pm 1.5$ \\
Circum. Strain (\%) & $-28.3 \pm 4.0$ & $-27.6 \pm 3.1$ & $-29.2 \pm 4.6$ \\
Longit. Strain (\%) & $-16.0 \pm 2.8$ & $-16.4 \pm 1.8$ & $-17.3 \pm 2.1$ \\
Area Strain (\%) & $-41.5 \pm 4.5$ & $-41.3 \pm 3.2$ & $-43.2 \pm 4.6$ \\
Radial Strain (\%) & $34.8 \pm 7.1$ & $32.5 \pm 7.5$ & $35.6 \pm 6.7$ \\
LV Torsion (\%/cm) & $2.14 \pm 0.85$ & $1.34 \pm 0.68+$ & $1.65 \pm 0.54$ \\
\hline
\end{tabular}

Abbreviations: see text (methods). Values are means $\pm S D+p<0.05$ versus baseline, $\# p<0.01$ versus baseline, * $p<0.05$ versus D3

\section{Geometry}

Both systolic and diastolic eccentricity were affected by altitude exposure, whilst LV sphericity remained unaltered (Figure $3 \mathrm{~A}$ and $3 \mathrm{~B}$ ). At baseline, LV geometry resembled a cone with a nearly circular cross-section, as reflected by a LV sphericity well under and an eccentricity close to unity both at end-diastole and end-systole. Both end-diastolic and end-systolic eccentricity values significantly increased at D3 and D4 but the difference between LV diastolic and systolic eccentricity at baseline (1.04 \pm 0.07 vs $1.00 \pm 0.06$, $\mathrm{p}<0.05$ ) disappeared at high altitude (Figure 3B), indicating that altitude exposure was associated with a mild, predominantly systolic ventricular D-shaping. Figures $3 \mathrm{C}$ and $3 \mathrm{D}$ display the effect of altitude exposure on the regional eccentricity as measured at the basal, midventricular and apical level. Although eccentricity increased numerically at every level, the effect could statistically be demonstrated most consistently at the midventricular level. 

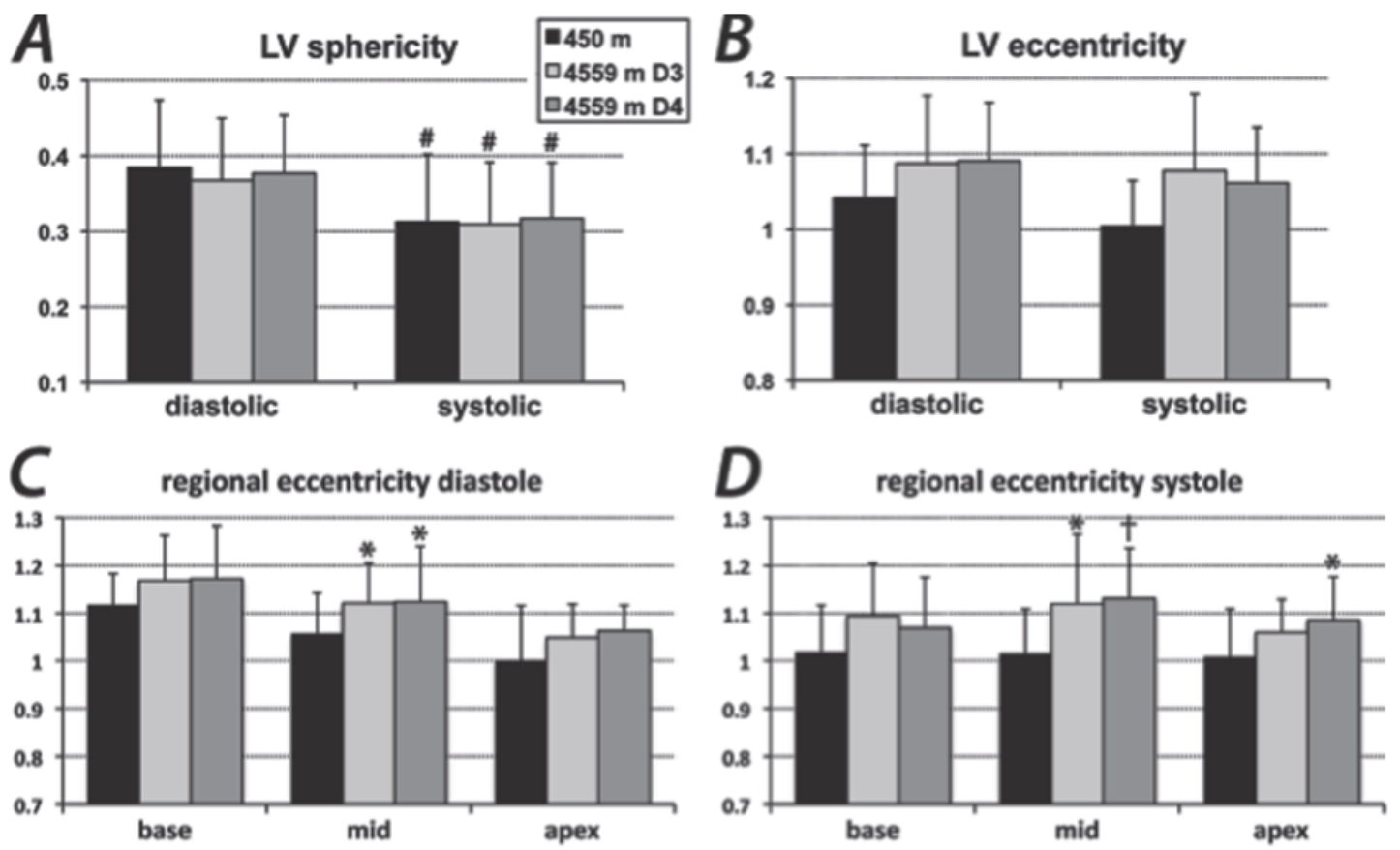

Figure 3. Global and local geometry in diastole and systole at baseline and altitude. Baseline at 450m is represented by black bars. D3 and D4 at $4559 \mathrm{~m}$ by light and dark gray bars respectively. \# $p<0.05$ versus systolic value at same altitude (not calculated for bottom panels). ${ }^{*} p<0.05$ versus baseline. $\dagger p<0.01$

\section{Interrelation between geometrical, torsional and haemodynamic changes}

Regression analysis demonstrated that the altitude-induced drop in $\mathrm{SpO} 2$ correlated with a rise in heart rate $(r=-0.517, p<0.001)$ and cardiac index $(r=-0.355, p=0.027)$. A positive relation to $\operatorname{LVEF}(r=0.378, p=0.018)$ and TAPSE $(r=0.342, p=0.035)$ was found despite their values on average remaining unchanged at altitude. Remarkably, no association between SpO2 and RVPGs could be demonstrated. However, the range of SpO2 (49-91\%) and RVPGs (27-58mmHg) at altitude was much larger than at baseline (Figure 2) and intra-individual values at D3 and D4 showed good agreement both for SpO2 ( $r=$ $0.658, p<0.001)$ and RVPGs $(r=0.757, p<0.001)$, suggesting that large inter-individual differences in response to altitude and hypoxia played a dominant role ${ }^{11,16}$. With regard to a potential direct relationship of $\mathrm{SpO} 2$ with either geometrical or torsional changes, no association reached statistical significance. Systolic ( $r=0.370, p=0.017)$ but not diastolic ( $r=0.241, p=0.130$ ) eccentricity significantly related to RVPGs.

Regarding torsional changes, regression analysis yielded weak, linear relations to changes in TAPSE ( $r=0.409, p=0.009)$ and $\operatorname{LVEF}(r=0.305, p=0.042)$, again despite average values of both the latter parameters remaining unchanged at altitude. In addition, a significant inverse relation was found between changes in torsion and in diastolic ( $r=-$ $0.329, p=0.032)$ and systolic $(r=-0.369, p=0.013)$ eccentricity. This relation between torsional changes and eccentricity remained significant after correcting for LVEF (diastolic $r=-0.319, p=0.035$; systolic $r=-0.336, p=0.026$ ) and TAPSE (diastolic $r=-0.337$, 
$p=0.035$; systolic $r=-0.401, p=0.009$ ). The strength of the relationship remained largely unaffected also after correcting for changes in RVPGs (diastolic $r=0.295, p=0.064$; systolic $r=0.371, p=0.018$ ), SpO2 (diastolic $r=-0.287, p=0.081$; systolic $r=-0.331, p=0.043$ ), heart rate (diastolic $r=-0.326, p=0.031$; systolic $r=-0.384, p=0.010$ ) and cardiac output (diastolic $r=-0.356, p=0.018$; systolic $r=-0.420, p=0.004$ ).

\section{DISCUSSION}

The main finding of the present study is that acute exposure to high-altitude leads to a decrease in LV torsion, which in turn relates to LV geometry, in particular to the extent of ventricular D-shaping.

Whereas previous studies explored twist in patients with a distorted LV geometry associated with long-standing alterations in ventricular structure, loading, and/or function, we investigated the relation between torsion and geometry by a paired analysis in healthy subjects, using a well-known short-term intervention (high-altitude exposure), and using 3D speckle tracking echocardiography. By substantiating that LV torsion decreases as LV eccentricity increases, and that it does so even in the absence of noticeable effects on LV structural remodelling, loading or pump function, our study suggests LV eccentricity to be an independent determinant of torsion.

\section{Geometry and torsional mechanics; comparison to previous studies}

A number of 2D speckle tracking studies have hinted towards a link between cardiac shape and torsional mechanics ${ }^{3-6,17}$. In particular, LV systolic twist has been reported to be impaired in the presence of chronic RV volume ${ }^{3,5,6}$ as well as pressure overload ${ }^{4}$, conditions typically associated with LV D-shaping. In patients with isolated atrial septum defects undergoing transcatheter closure, Dong and colleagues found an immediate restoration of twist mechanics ${ }^{3}$ after relief of RV volume overload. Furthermore, in patients with persistent RV dilatation and dysfunction after surgical correction of tetralogy of Fallot, impaired LV twist was related both to increased LV eccentricity ${ }^{6}$ as well as to reduced RV free wall function ${ }^{5}$. Finally, an inverse relationship between twist and LV eccentricity has been demonstrated also in a study by Puwanant comparing normal controls to patients with RV dilatation and RV dysfunction due to chronic pulmonary hypertension ${ }^{4}$.

Whereas our study confirms the inverse relation between LV eccentricity and torsional mechanics, a fundamental difference is that our study involved healthy subjects, a pairwise comparison, and an intervention brief and mild enough to avoid remodelling and relevant LV loading alterations. Indeed, chronic pulmonary hypertension as in the study of Puwanant, is associated with a significant decline in LV enddiastolic and stroke volumes, as well as impaired LV diastolic filling ${ }^{4,18}$. This is important, as LV torsion is 


\section{CHAPTER 6}

loading dependent, with lower LV end-diastolic volumes producing lower LV torsion ${ }^{19}$. The preserved LVEDV, LV-SV, as well as E/A ratio and E/E' in both the present and previous studies suggest that pulmonary hypertension induced at such an altitude is not associated with measurable decreases in stroke volume or changes in diastolic filling markers ${ }^{16,20,21}$. In addition, in most of the mentioned studies, some kind of RV dysfunction and/or remodeling was documented. Chronic RV overload may instigate gradual RV and septal remodeling, eventually leading to deterioration of contraction and structure beyond that explained by altered loading itself ${ }^{22}$. The short study duration and the paired analyses in healthy subjects practically exclude such structural and contractile remodeling effects as potential confounders in our observations. In fact, considering the elevated afterload (RVPGs), the preserved RV-FAC and TAPSE and mildly increased annular tissue Doppler velocities even suggest RV function to be enhanced during short-term RV pressure overload at high altitude ${ }^{21,23}$. Thus, our data established an inverse relation between eccentricity and torsion over a relatively large range of mostly mild PH without remodeling, RV dysfunction or relevant LV loading changes as potential confounders.

\section{Effects of altitude exposure on hemodynamics and biventricular geometry}

The primary mechanism underlying $\mathrm{PH}$ at altitude is hypoxia-induced arteriolar vasoconstriction. Exposure to an altitude of $4559 \mathrm{~m}$, equivalent to an inspired oxygen concentration of roughly half $(\approx 0.12)$ that at sea level $(0.21)$, augments systolic pulmonary pressure to an average of about $40 \mathrm{mmHg}$, typically with a considerable interindividual variability ${ }^{16}$ also seen in the present study. During initial altitude exposure, oxygen delivery at rest is maintained constant by an adrenergic surge that raises both heart rate and cardiac output by roughly $20 \%$ without affecting LV filling, LVEF and stroke volume ${ }^{16,21,24,25}$. Importantly, altitude-induced PH occurs without any alteration in pulmonary artery wedge pressure ${ }^{16}$. The increases in heart rate, cardiac output and pulmonary pressures observed in our study are in that same range without evidence of reduced LV stroke volume or LV preload.

The increased LV eccentricity in response to altitude-induced PH is similar to other conditions associated with acute and chronic $\mathrm{PH}^{4,12,21}$. RV adaptation to pressure overload typically involves a change in geometry from a crescent to a more spherical configuration with increased diastolic and systolic areas and volumes ${ }^{26}$. Increased RV pressures and RV dilatation cause a decreased transseptal pressure gradient with flattening and displacement of the IVS towards the LV ${ }^{18,27,28}$, resulting in D-shaping of the left ventricle ${ }^{15,22}$. The fact that altitude-induced PH mainly affects systolic RV pressures probably explains why this effect was most evident for systolic eccentricity in our study ${ }^{27}$. 


\section{Potential explanations for the inverse relation between torsion and LV eccentricity}

The exact mechanisms relating eccentricity to torsion in the present study remain elusive. Torsion is most commonly ascribed to the interaction between epicardial and endocardial fibre sheets running at oblique angles within the myocardial wall. These two fibre families produce rotational forces with opposite moments. For the generation of a net torsion, a mechanical advantage of the epicardial fibers over the endocardial fibers is therefore required ${ }^{1}$. This mechanical advantage is commonly attributed to the superior radius and thus leverage of subepicardial relative to subendocardial fibers, although their relative angles and transmural gradients in their electrical activation, loading and contractility also have been ascribed a role ${ }^{1,29}$. Interestingly, not only multidirectional strains but also LV ejection fraction remained unaffected in our study despite decreased torsion, potentially indicating that rather than a diminished mechanical interaction between the epicardial and endocardial fibers itself, the effect of geometry on torsion involves alterations in epicardial vs endocardial loading and/or torque angle through septal flattening ${ }^{29}$. It is conceivable that the geometric distortion associated with septal flattening leads to a change in the arrangement of myocardial fibers. Also, septal flattening reduces the torque angle difference between the right and left sided septal fibers. Thus, a combined effect of relative unloading of the septal "epicardial" fibers and equalisation of epi- and endocardial radii may play an important role. In computer models Gibbons-Kroeker suggested that the effect of such abnormal bending forces at the septum may extend into to the free wall as well ${ }^{28}$. They found major distortions particularly at the RV insertion points, where left and right ventricular fibers intertwine and fibrosis occurs in chronic disease. This may in part explain the link between RVfunction and LV torsion found in most studies ${ }^{3,5,6}$.

\section{Study limitations}

Previous studies used 2D imaging and speckle tracking to determine LV twist and geometry. As within the LV volume both regional shape as well as rotation progressively vary from base to apex, proper standardisation of the images planes and/or correcting for distance between two measurement is mandatory when comparing values within and between subjects ${ }^{30}$. 3D speckle tracking echocardiography has major advantages in this regard, but inherently suffers from lower sampling rates and image quality. This precludes a judicious analysis of (un-)twisting rates in a study with a limited size like ours. In addition, even when employing a single observer and standardized method, measurement variability of LV torsion was high. As bias was negligible and measurement variability only a fraction of the observed intervention-effect, we believe this issue to have introduced noise rather than spurious observations. 


\section{CHAPTER 6}

Uncertainties remain in particular regarding right-sided filling pressures, and thus on the potential effect of diastolic transseptal pressure gradients on the observed LV eccentricity and torsional changes at altitude. We did not notice changes in hepatic vein flow patterns or inferior caval vein collapsibility in our study. On the other hand, considering the elevated RV diastolic (and systolic) areas at altitude it appears that the RV reacted to increased afterload with augmentation of its operating volumes, i.e. by preload recruitment ${ }^{21}$.

\section{CONCLUSION}

Altitude-induced pulmonary hypertension and LV D-shaping reduced LV torsion even in the absence of noticeable effects on LV loading and biventricular pump function. This effect was appreciated in a time frame too short to induce structural remodeling. Changes in torsion were inversely related to LV eccentricity (D-shaping) even after correcting for other parameters relating to torsion, suggesting a direct relation between LV shape and torsional mechanics. 


\section{ALTERED LEFT VENTRICULAR GEOMETRY AND TORSIONAL MECHANICS ...}

\section{REFERENCES}

1. Sengupta PP, Tajik AJ, Chandrasekaran K, Khandheria BK. Twist mechanics of the left ventricle: principles and application. JACC Cardiovasc Imaging 2008; 1(3):366-376.

2. Rademakers FE, Rogers WJ, Guier WH, Hutchins GM, Siu CO, Weisfeldt ML, Weiss JL, Shapiro EP. Relation of regional cross-fiber shortening to wall thickening in the intact heart. Three-dimensional strain analysis by NMR tagging. Circulation 1994; 89(3):1174-1182.

3. Dong L, Zhang F, Shu X, Zhou D, Guan L, Pan C, Chen H. Left ventricular torsional deformation in patients undergoing transcatheter closure of secundum atrial septal defect. Int J Cardiovasc Imaging 2009; 25(5):479-486.

4. Puwanant S, Park M, Popovic ZB, Tang WH, Farha S, George D, Sharp J, Puntawangkoon J, Loyd JE, Erzurum SC, Thomas JD. Ventricular geometry, strain, and rotational mechanics in pulmonary hypertension. Circulation 2010; 121(2):259-266.

5. van der Hulst AE, Delgado V, Holman ER, Kroft LJ, de Roos A, Hazekamp MG, Blom NA, Bax JJ, Roest AA. Relation of left ventricular twist and global strain with right ventricular dysfunction in patients after operative "correction" of tetralogy of fallot. Am J Cardiol 2010; 106(5):723-729.

6. Cheung YF, Wong SJ, Liang XC, Cheung EW. Torsional mechanics of the left ventricle in patients after surgical repair of tetralogy of Fallot. Circ J 2011; 75(7):1735-1741.

7. Mor-Avi V, Jenkins C, Kuhl HP, Nesser HJ, Marwick T, Franke A, Ebner C, Freed BH, SteringerMascherbauer R, Pollard H, Weinert L, Niel J, Sugeng L, Lang RM. Real-time 3-dimensional echocardiographic quantification of left ventricular volumes: multicenter study for validation with magnetic resonance imaging and investigation of sources of error. JACC Cardiovasc Imaging 2008; 1(4):413-423.

8. Nesser HJ, Mor-Avi V, Gorissen W, Weinert L, Steringer-Mascherbauer R, Niel J, Sugeng L, Lang RM. Quantification of left ventricular volumes using three-dimensional echocardiographic speckle tracking: comparison with MRI. Eur Heart J 2009; 30(13):1565-1573.

9. Tanaka H, Hara H, Saba S, Gorcsan J, 3rd. Usefulness of three-dimensional speckle tracking strain to quantify dyssynchrony and the site of latest mechanical activation. Am J Cardiol 2010; 105(2):235-242.

10. De Boeck BW, Cramer MJ, Oh JK, van der Aa RP, Jaarsma W. Spectral pulsed tissue Doppler imaging in diastole: a tool to increase our insight in and assessment of diastolic relaxation of the left ventricle. Am Heart J 2003; 146(3):411-419.

11. Allemann Y, Sartori C, Lepori M, Pierre S, Melot C, Naeije R, Scherrer U, Maggiorini M. Echocardiographic and invasive measurements of pulmonary artery pressure correlate closely at high altitude. Am J Physiol Heart Circ Physiol 2000; 279(4):H2013-2016.

12. Rudski LG, Lai WW, Afilalo J, Hua L, Handschumacher MD, Chandrasekaran K, Solomon SD, Louie EK, Schiller NB. Guidelines for the echocardiographic assessment of the right heart in adults: a report from the American Society of Echocardiography endorsed by the European Association of Echocardiography, a registered branch of the European Society of Cardiology, and the Canadian Society of Echocardiography. J Am Soc Echocardiogr 2010; 23(7):685-713; quiz 786-688.

13. Cerqueira MD, Weissman NJ, Dilsizian V, Jacobs AK, Kaul S, Laskey WK, Pennell DJ, Rumberger JA, Ryan T, Verani MS. Standardized myocardial segmentation and nomenclature for tomographic imaging of the heart: a statement for healthcare professionals from the Cardiac Imaging Committee of the Council on Clinical Cardiology of the American Heart Association. Circulation 2002; 105(4):539-542.

14. Mannaerts HF, van der Heide JA, Kamp O, Stoel MG, Twisk J, Visser CA. Early identification of left ventricular remodelling after myocardial infarction, assessed by transthoracic 3D echocardiography. Eur Heart J 2004; 25(8):680-687.

15. Ryan T, Petrovic O, Dillon JC, Feigenbaum H, Conley MJ, Armstrong WF. An echocardiographic index for separation of right ventricular volume and pressure overload. J Am Coll Cardiol 1985; 5(4):918-927.

16. Maggiorini M, Melot C, Pierre S, Pfeiffer F, Greve I, Sartori C, Lepori M, Hauser M, Scherrer U, Naeije R. High-altitude pulmonary edema is initially caused by an increase in capillary pressure. Circulation 2001; 103(16):2078-2083. 


\section{CHAPTER 6}

17. van Dalen BM, Kauer F, Vletter WB, Soliman OI, van der Zwaan HB, Ten Cate FJ, Geleijnse ML. Influence of cardiac shape on left ventricular twist. J Appl Physiol 2010; 108(1):146-151.

18. Lumens J, Blanchard DG, Arts T, Mahmud E, Delhaas T. Left ventricular underfilling and not septal bulging dominates abnormal left ventricular filling hemodynamics in chronic thromboembolic pulmonary hypertension. Am J Physiol Heart Circ Physiol 2010; 299(4):H1083-1091.

19. Weiner RB, Weyman AE, Khan AM, Reingold JS, Chen-Tournoux AA, Scherrer-Crosbie M, Picard MH, Wang TJ, Baggish AL. Preload dependency of left ventricular torsion: the impact of normal saline infusion. Circ Cardiovasc Imaging 2010; 3(6):672-678.

20. Bernheim AM, Kiencke S, Fischler M, Dorschner L, Debrunner J, Mairbaurl H, Maggiorini M, Brunner-La Rocca HP. Acute changes in pulmonary artery pressures due to exercise and exposure to high altitude do not cause left ventricular diastolic dysfunction. Chest 2007; 132(2):380-387.

21. Huez S, Faoro V, Guenard H, Martinot JB, Naeije R. Echocardiographic and tissue Doppler imaging of cardiac adaptation to high altitude in native highlanders versus acclimatized lowlanders. Am J Cardiol 2009; 103(11):1605-1609.

22. Bristow MR, Zisman LS, Lowes BD, Abraham WT, Badesch DB, Groves BM, Voelkel NF, Lynch DM, Quaife RA. The pressure-overloaded right ventricle in pulmonary hypertension. Chest 1998; 114(1 Suppl):101S$106 \mathrm{~S}$.

23. Allemann Y, Stuber T, de Marchi SF, Rexhaj E, Sartori C, Scherrer U, Rimoldi SF. Pulmonary artery pressure and cardiac function in children and adolescents after rapid ascent to 3,450 m. Am J Physiol Heart Circ Physiol 2012; 302(12):H2646-2653.

24. Naeije R. Physiological adaptation of the cardiovascular system to high altitude. Prog Cardiovasc Dis 2010; 52(6):456-466.

25. Klausen K. Cardiac output in man in rest and work during and after acclimatization to 3,800 m. J Appl Physiol 1966; 21(2):609-616.

26. Cho EJ, Jiamsripong P, Calleja AM, Alharthi MS, McMahon EM, Khandheria BK, Belohlavek M. Right ventricular free wall circumferential strain reflects graded elevation in acute right ventricular afterload. Am J Physiol Heart Circ Physiol 2009; 296(2):H413-420.

27. Lee JM, Boughner DR. Tissue mechanics of canine pericardium in different test environments. Evidence for time-dependent accommodation, absence of plasticity, and new roles for collagen and elastin. Circ Res 1981; 49(2):533-544.

28. Gibbons Kroeker CA, Adeeb S, Tyberg JV, Shrive NG. A 2D FE model of the heart demonstrates the role of the pericardium in ventricular deformation. Am J Physiol Heart Circ Physiol 2006; 291(5):H2229-2236.

29. Evangelista A, Nardinocchi P, Puddu PE, Teresi L, Torromeo C, Varano V. Torsion of the human left ventricle: experimental analysis and computational modeling. Prog Biophys Mol Biol 2011; 107(1):112-121.

30. van Dalen BM, Vletter WB, Soliman OI, ten Cate FJ, Geleijnse ML. Importance of transducer position in the assessment of apical rotation by speckle tracking echocardiography. J Am Soc Echocardiogr 2008; 21(8):895-898. 


\section{CHAPTER 7}

Discussion, Future Directions and Valorisation 


\section{STATE OF THE ART ECHOCARDIOGRAPHY IN CLINICAL RESEARCH}

Clinical echocardiography has seen a continuous technical development over the last 5 decades, and echocardiography is today by far the most utilized imaging technique in cardiology. At the same time, echocardiography has contributed a great deal to our understanding of cardiac physiology and pathophysiology. Novel technical developments in echocardiography can often be utilized to measure specific parameters with more reliability, or to assess parameters that were not readily measurable without these technical developments.

However, in order to turn the use of echocardiography in a specific research question into meaningful science, several caveats apply. First, one needs to have a thorough understanding of the knowns and unknowns of the physiologic or pathophysiologic process that will be studied. Second, a correct understanding of what exactly and how a specific echocardiographic technique will measure is mandatory. Nowhere is this better exemplified than in the assessment of myocardial perfusion with ultrasound contrast agents, where an understanding of the complex regulation of myocardial blood flow, of the properties of ultrasound contrast agents and of ultrasound physics are necessary. Last, one needs to keep in mind the limitations of ultrasound imaging. The most important limitation is the stochastic nature of ultrasound when compared to other imaging techniques like CT or MRI. This and a number of known artefacts can lead to variability in the collected data. However, given the wide availability, ease of use and portability of ultrasound equipment, and the ever increasing image quality, these limitations are by far outweighed not just in clinical practice, but also in research applications.

\section{MYOCARDIAL BLOOD FLOW RESERVE MEASURED WITH MYOCARDIAL CONTRAST ECHOCARDIOGRAPHY}

In order to deliver oxygen and nutrients to the myocardium, blood flows through the coronary arteries, then arterioles, the myocardial capillaries, drains into myocardial venules and veins and finally the right atrium. The flow of blood through the myocardium is determined by the pressure gradient across the vascular bed, also termed coronary driving pressure (CDP) and the resistance (myocardial vascular resistance, MVR) offered by the myocardial vascular system. In a normal vasculature at rest and assuming a mean aortic pressure of about $90 \mathrm{mmHg}$, the CDP is approximately $80-85 \mathrm{mmHg}$. Normal epicardial coronay arteries offer almost no resistance to blood flow. However, the pressure is reduced to pre-capillary levels of approximately $45 \mathrm{mmHg}$ by myocardial arterioles; this reduction in pressure is crucial, because a higher hydrostatic pressure in the myocardial capillaries would lead to fluid movement out of the capillary bed into the myocardium and result in tissue edema. Thus, at rest about 60 percent of the total MVR is offered by arterioles. Myocardial capillaries are about $0.15 \mathrm{~mm}$ long and their 
diameter is about $7 \mu \mathrm{m}$, and thus individual capillaries would offer a high resistance ${ }^{1}$. However, the 1.5 billion capillaries present in the human myocardium are laid out in parallel, and therefore at rest only about $25 \%$ of MVR is offered by the capillaries, leading to mean capillary pressure of about $30 \mathrm{mmHg}$ and a post-capillary pressure of about $15 \mathrm{mmHg}$. Finally, about $15 \%$ of total MVR resistance is offered by the venules ${ }^{2}$.

During exercise, myocardial oxygen demand increases. As oxygen extraction is about $60-70 \%$ at rest and the cardiac venous oxygen content is kept constant during exercise, myocardial blood flow is tightly coupled to oxygen consumption ${ }^{3}$ to meet the increased demand. This increase in myocardial blood flow is mainly brought about by a decrease in arteriolar resistance ${ }^{2}$. The rapid increase in blood flow in response to exercise is controlled by a variety of factors. Carbon dioxide and reactive oxygen species are produced in proportion to myocardial oxygen consumption and function in a feed-forward manner to increase myocardial blood flow ${ }^{4,5}$. In addition, tissue hypoxia, adenosine and nitric oxide (NO) ${ }^{6-8}$ seem to play a role.

In subjects that develop HAPE upon ascent to altitude, endothelial dysfunction is thought to lead to exaggerated hypoxic vasoconstriction ${ }^{9}$. However, there are data that indicate that this endothelial dysfunction is not limited exclusively to the pulmonary circulation, but rather can be found as well in the systemic circulation ${ }^{10}$. We therefore set out to answer the question, whether the measurement of myocardial blood flow reserve in HAPE susceptible subjects compared to controls would show an endothelial dysfunction in the coronary circulation as well (Chapter 2). Our data show that myocardial blood flow reserve is impaired in HAPE susceptible subjects at high altitude ${ }^{11}$. Interestingly, we also showed that in HAPE susceptibles that are treated with either dexamethasone or tadalafil to prevent HAPE, myocardial blood flow reserve is not reduced at high altitude. Both drugs are thought to promote vasodilation either by an increase in the production of NO in the pulmonary vasculature (dexamethasone), or by potentiating the effect of NO by inhibiting the breakdown of cGMP (tadalafil) ${ }^{12,13}$. Thus, while the

role of NO is somewhat controversial ${ }^{3}$, our data argue for a role of NO in the regulation of myocardial flow when the human organism is exposed to hypoxia, as occurs at high altitude.

\section{ASSESSMENT OF CARDIAC CHAMBER SIZES AND EJECTION FRACTION}

In nearly every cardiac disease, the size of the heart chambers can be altered, and the left atrium is no exception. While the left atrium could be viewed simply as a chamber receiving oxygenated blood from the pulmonary veins, in fact its function is far more complex. During ventricular systole, the left atrium functions as a reservoir that receives blood from the lungs, while its volume is enlarged as the mitral annulus displaces towards the left ventricular apex. After mitral valve opening, the left atrium serves as a conduit carrying blood directly from the pulmonary veins into the left ventricle. Finally, 
in late diastole the left atrium contracts and creates a pressure gradient and thus a flow from the atrium into the left ventricle. The atrial contraction in late diastole is also called "atrial kick" and is thought to contribute directly to left ventricular function ${ }^{14}$. Obviously, the structure and function of the left atrium is closely related to abnormalities of the left ventricle. Thus, decreased left ventricular ejection fraction or left sided valvular disease often lead to increased left atrial filling pressure and consequently to left atrial enlargement ${ }^{15}$. Initially, left atrial enlargement leads to an improvement in atrial function. However, similar to the Frank Starling curve of the left ventricle, left atrial function declines, once the volume has surpassed a certain threshold ${ }^{14}$. As left atrial enlargement reflects left ventricular filling pressures over time, left atrial volume measurements have also been compared to the measurement of $\mathrm{HbA1C}$ in diabetes ${ }^{16}$, 17. Left atrial volume is predictive of cardiovascular events such as atrial fibrillation, stroke, heart failure and death in a wide array of populations such as after a myocardial infarction ${ }^{18}$, heart failure ${ }^{19}$, atrial fibrillation ${ }^{20}$, after cardiac surgery ${ }^{21}$, but notably also in community based populations ${ }^{22}$. Over decades, measurement of the anteroposterior diameter has been used to assess enlargement of the left atrium. However, left atrial dilatation often occurs often more along the long axis of the atrium, and may be missed when only the antero-posterior diameter is measured. Left atrial volumes can be derived from 2-dimensional images either using an ellipsoid model or the Simpson's method, and assessment with the latter has been shown to be superior over onedimensional measurements for predicting adverse cardiac outcomes ${ }^{23}$. 3-dimensional echocardiography has also been used for the measurement of left atrial volumes, and compares favourably to 2-dimensional echocardiography regarding test-retest variability and agreement to other imaging methods like magnetic resonance imaging ${ }^{24,} 25$. However, these studies used software that was developed for measuring left ventricular volumes on 3-dimensional datasets. We therefore evaluated a novel software tool (TomTec Imaging Systems) specifically developed for the assessment of left atrial volumes (chapter 4). This software creates a polyhedron model of the LA using an automated border-detection technique. We could show that the dedicated software outperformed software that was not specifically developed for measuring left atrial volumes in terms of accuracy compared to magnetic resonance imaging ${ }^{26}$. While the measurement of left atrial volumes was somewhat more time-consuming using the 3-dimensional software than with 2-dimensional software, in terms of clinical applicability this was not relevant. Given the prognostic value of left atrial volumes, this additional time seems worthwhile given the increased accuracy and reliability. 3-dimensional echocardiography also offers a relatively easy possibility to assess left atrial volumes throughout the cardiac cycle, and thus likely will yield a more detailed insight into left atrial function in the future.

The single number most frequently asked from an echocardiographic study is left ventricular ejection fraction. Left ventricular ejection fraction has been used as a cut-off value for the indication of drug therapy ${ }^{27}$ and device implantation ${ }^{28,29}$, and also in the 
decision on whether to surgically intervene in valve disease ${ }^{30}$. The accuracy of measurements of left ventricular ejection fraction has typically been investigated in controlled trials in well-characterised, selected patient groups ${ }^{31}$. While newer methods like left ventricular opacification and 3-dimensional echocardiography clearly improve accuracy and reproducibility ${ }^{31,32}$, assessment of left ventricular ejection fraction by the Simpson's biplane method or even by simple eyeballing is clearly a reality in everyday clinical practice even today. While such techniques may be adequate to detect even small differences in left ventricular ejection fraction in large intervention trials ${ }^{33,34}$, low reproducibility may have a significant impact on clinical decision making in individual patients. In a large heart-failure patient cohort we compared left ventricular ejection fraction measurements made on-site at the recruiting centers to measurements made at our core laboratory using Simpson's biplane method (Chapter 3) ${ }^{35}$. While as expected, we found good overall correlations, there was a high variability in individual patients. In a retrospective analysis using well-accepted cut-off values used for indicating the use of implantable cardioverter-defibrillators, about $20 \%$ of patients crossed either from having an indication (ejection fraction below cut-off) to having no indication (ejection fraction above cut-off) when comparing measurements from the recruiting center versus the core laboratory. Thus, our data are clearly a call for the more widespread use of better quantitative methods like the use of contrast agents or 3dimensional echocardiography for the measurement of left ventricular ejection fraction.

\section{GUIDED TREATMENT OF HEART FAILURE - EFFECT ON CARDIAC REMODELING}

Brain natriuretic peptide (BNP) is released by the cardiomyocytes in response to mechanical strain such as occurs in pressure overload ${ }^{36}$. BNP is synthesized as a 108 amino acid precursor which is proteolytically cleaved to the biologically active 32 amino acid BNP and the 76 amino acid NT-proBNP which is biologically inactive. As the half-life of BNP is about 20 minutes, whereas the half-life of NT-proBNP is $90-120$ minutes. Thus, measured serum values are lower for BNP than for NT-proBNP. The biological effects of BNP counteract the adverse effects of decompensated heart failure. Thus, BNP has a natriuretic and diuretic effect, downregulates the renin-angiotensin system, and leads to vasodilation ${ }^{37}$.

The rapid elevation of BNP in response to increased wall tension has been used as a diagnostic tool to evaluate patients presenting to the emergency room with acute dyspnea. It has been shown that the measurement of BNP provides incremental diagnostic benefit over historical or physical findings in separating patients with acute dyspnea due to cardiac disease from patients with noncardiac causes of dyspnea ${ }^{38}$. Thus, measurement of BNP in patients suspected of having acute heart failure is currently endorsed by clinical practice guidelines ${ }^{39}$. 
When heart failure patients in acute decompensation are treated with loop diuretics and ACE inhibitors, BNP levels decrease as a reflection of decreasing left ventricular filling pressures ${ }^{40}$. Thus, another area where measurement of BNP levels has been examined is in guiding heart failure treatment. Several randomized controlled trials have been conducted in this area with mixed results. In general, younger patients with lower achieved values of BNP experienced a benefit of BNP-guided uptitrating heart failure medication ${ }^{41}$, , whereas this effect was not evident in the elderly, or when reductions in BNP were not substantial ${ }^{43}$. The Trial of Intensified versus standard Medical therapy in Elderly patients with Congestive Heart Failure (TIME-CHF) ${ }^{44}$ included 499 patients 60 years or older with systolic heart failure defined as a left ventricular ejection fraction $\leq 45 \%$. In addition, the patients had to been at least in New York Heart Association (NYHA) class II, and had to hospitalized for heart failure within the year prior ton inclusion and to have a NT-proBNP level twice the upper limit of normal. Of note, TIME$\mathrm{CHF}$ included many patients with an age $\geq 75$ years, and thus is representative of a realworld heart failure population. The patients were randomized into a symptom-guided and a NT-proBNP-guided group. In the symptom-guided group, heart failure medication was uptitrated with the goal of reducing symptoms to NYHA class II or less. In the NTproBNP guided group, medication was uptitrated with the goal of reducing NT-proBNP levels to twice the upper limit of normal or less, and symptoms to NYHA II or less. Overall, in the study population, NT-proBNP guided treatment did not reduce survival free of hospitalizations at 18 months, nor did it improve quality of life. However, in the prespecified group of $<75$ years old patients, hospital free survival and even overall free survival was reduced.

An important factor in the prognosis of patients treated for heart failure with reduced ejection fraction is the reversal of cardiac remodeling ${ }^{45,46}$. In a substudy of the TIME-CHF, we investigated the evolution of left ventricular ejection fraction and left ventricular volumes in patients in the symptom-guided versus the NT-proBNP guided groups (chapter 5). Overall, left ventricular ejection fraction increased and both enddiastolic and end-systolic volumes decreased in the whole study population. The magnitude of the increases in left ventricular ejection fraction that we observed was with an absolute 10 percentage points surprisingly large, far larger than in drug intervention trials in heart failure ${ }^{46-48}$. In addition, the assignment to a NT-proBNP guided treatment strategy significantly and favourably interacted with the increase in left ventricular ejection fraction. This effect was independent of age, and was also present in patients $\geq 75$ years. Our data thus shed some light on the difficulties faced when treating elderly heart failure patients, which is the large and growing population. The fact that improvements in cardiac functional parameters do not translate into better clinical outcomes as is the case in the younger patients, suggests that other factors such as comorbidities play a more important role in the elderly. 


\section{LEFT VENTRICULAR TORSION AT HIGH ALTITUDE}

Myocardial fibers in the left ventricle are arranged obliquely relative to the long axis. Fibers in the subendocardium spiral around the long axis right-handed, whereas the orientation of subepicardial fibers is left handed. When viewed from the apex towards the base, this leads to a predominantly clockwise rotation of the base of the heart, and a counterclockwise rotation of the apex during systolic contraction, producing a wringing motion of the left ventricle ${ }^{49}$. Several cardiac diseases have an impact on left ventricular torsion, such as pressure overload in aortic stenosis ${ }^{50}$, ischemic heart disease ${ }^{51}$, dilated cardiomyopathy ${ }^{52}$ and diabetes ${ }^{53}$. Left ventricular geometry may be one important determinant of left ventricular torsion, and in fact this has been investigated in several studies. However, the drawback of these studies was that the influence of geometry on torsion was investigated in patient populations with cardiac or pulmonary disease, which may affect torsion independent of geometry ${ }^{54,55}$. Pulmonary pressure induced septal flattening in healthy subjects that are exposed to high altitude offer a unique possibility to study the influence of changes in left ventricular geometry on torsion mechanics. As one of the disadvantages of 2-dimensional speckle tracking echocardiography for the assessment of torsion is out-of plane motion through the cardiac cycle ${ }^{56}$, we used 3-dimensional speckle tracking echocardiography to assess the influence of changes in left ventricular geometry on torsion in healthy subjects rapidly ascending to 4550 meters above sea level (chapter 6). We could show that the increase in pulmonary artery pressure that occurs at high altitude leads to left ventricular Dshaping which in turn caused a decrease in left ventricular torsion. As these subjects were healthy and the time spent at high altitude was too short for left ventricular remodeling to occur, we could establish a direct relationship between left ventricular geometry and torsion.

\section{CONCLUSIONS AND FUTURE DIRECTIONS}

In this thesis, we have used state-of-the art echocardiography to improve knowledge of left ventricular and left atrial structure, physiology and function. In individuals exposed to high altitude we showed a reduced myocardial blood flow reserve in subjects developing high altitude pulmonary edema (chapter 2). This points to a reduced endothelial function in the myocardial microcirculation in response to hypoxia at high altitude in susceptible individuals. These findings raise the important question whether at low altitude there are also subsets of the population that react differently to hypoxia which can occur in critical disease, and whether such differences have prognostic implications. Measurements of blood flow reserve using myocardial contrast echocardiography could in the future possibly contribute to assessing the microcirculatory function and its prognostic implication in patients with critical disease with hypoxia. In a similar patient 
population exposed to high altitude we used 3-dimensional echocardiography to assess torsional left ventricular mechanics, and could show that hypoxia induced pulmonary artery hypertension and the accompanying left ventricular D-shaping leads to decreases in the extent of torsion (chapter 6). These data are important for understanding the effect of changes in left ventricular geometry on torsion. Pulmonary artery hypertension due to diseases at low altitude such as chronic obstructive pulmonary disease or pulmonary embolism often goes hand in hand with pathophysiologic effects that impact left ventricular function independently. In contrast, high altitude exposure is an ideal model to study the physiologic effects of changes in left ventricular geometry on torsion without the confounding effects of diseases that lead to elevated pulmonary artery pressure at low altitude. 3-dimensional echocardiography was also used to assess left ventricular volumes and validate a novel software tool against the gold standard magnetic resonance (chapter 4) and could show that echocardiographic 3-dimensional measurements are more accurate than 2-dimensional echocardiography. Thus, future studies should assess whether more accurate measurements of left atrial volumes could aid in patient risk stratification - not just in heart failure, but possibly also in valvular heart disease such as mitral regurgitation. In a large heart failure population, we used echocardiography to assess left ventricular function and remodeling (chapters 3 and 5). As a caveat regarding 2-dimensional echocardiography, we found a relatively high variability in the assessment of the left ventricular ejection fraction with potential implications on the selection of patients for therapies such as implantation of implantable cardioverter defibrillators (ICD). Future studies will have to assess whether technical developments such as left ventricular opacification using contrast agents or 3dimensional measurements of left ventricular function decreases variability and results in less patients in which there are uncertainties regarding the indications of therapies like ICDs. Finally, in the same heart failure cohort we assessed the influence of NTproBNP-guided versus symptom-guided intensification of heart failure therapy. We found an increase in left ventricular ejection fraction that was larger in the patients with NT-proBNP-guided therapy. This was true both for patients $<75$ as well as patients $\geq 75$ years of age, whereas an improvement in clinical outcome measures was present only in the younger patients. This may be due to more comorbidities, and larger studies dedicated to elderly patients may be necessary to show a potential beneficial effect of NT-proBNP-guided therapy, and a multicenter trial that will hopefully answer this question is currently underway ${ }^{57}$. Thus, in summary in this thesis we have used various echocardiographic techniques to improve the understanding of cardiac physiology and structure in patients with heart failure and in healthy volunteers exposed to high altitude. 


\section{VALORISATION}

Echocardiography has developed into an imaging technique that is indispensable in the current practice of cardiology. Research that covers all aspects of modern echocardiography continues to be highly relevant in a wide range of areas.

First, the performance of existing techniques such as the assessment of left ventricular ejection fraction using either visual assessment or the Simpson's biplane method has often been validated in relatively selected patient population. Therefore, it is important to assess the performance of these techniques also in larger populations that more closely resemble populations that are typically encountered in everyday clinical practice. The data presented in chapter 3 of this thesis show that conventional assessment of left ventricular ejection fraction is fraught with a considerable variability in an elderly heart failure population. The data also show that this can potentially have a significant impact on clinical decision making such as in whom to implant an ICD. These data are relevant on several levels. Of course, in the light of these data, research in echocardiography should be directed towards developing techniques for more reliable assessment of left ventricular ejection fraction that are applicable in everyday clinical practice. In addition, both cardiologists as well as internists caring for elderly heart failure patients need to be aware of the limitations when measuring left ventricular ejection fraction. These considerations are also relevant on a socio-economic level when considering the use of costly therapies such as ICDs.

Second, existing techniques can be used to assess the effect of interventions in specific patient populations. In chapter 5, we have used echocardiography to assess the effect of intensified heart failure therapy on left ventricular function. We show that, overall, intensification in heart failure therapy leads to a significant improvement in left ventricular ejection fraction, and that guidance of therapy with the use of the biomarker NT-pro-BNP has a positive impact on this improvement. These data are of interest to heart failure specialists, and will also be of value when designing future intervention trials in heart failure.

Third, novel software tools that assist in measuring cardiac dimensions on imaging datasets need to be validated in terms of their performance against a gold standard and in terms of ease of use. In chapter 4 we compare conventional methods and a novel software tool for measuring left atrial volumes against magnetic resonance imaging. We show that compared to magnetic resonance imaging, the novel software tool is more accurate but also more time-consuming. These data are relevant not only to cardiologists who need to interpret cardiac imaging studies, but also to engineers and software developers. In addition, this novel and more accurate tool could potentially be used in future studies that assess the prognostic value of left atrial size for example in patients with paroxysmal atrial fibrillation.

Fourth, in chapter 1 and chapter 6 we use novel technologies (myocardial contrast echocardiography for the assessment of myocardial perfusion, 3-dimensional speckle 


\section{CHAPTER 7}

tracking for the measurement of left ventricular torsion) to asses (patho-)physiologic changes that occur during adaptation to high altitude. While at first glance high-altitude physiology might seem to be a minor health issue, it should be noted that (a) large populations in the Andes and Himalayas are living at high altitude, and (b) people increasingly travel to high altitudes. Thus, there is a growing interest in understanding the mechnisms of adaptation to high altitude both in healthy subjects and in subjects that are prone to develop high altitude. Thus, the data in chapters 1 and 6 are of interest to researchers and health care professionals dealing with people who intend to travel to high altitudes. In addition, understanding of the mechanisms of adaptation to high altitude with profound hypoxia may also shed light on the response of the human cardiocirculatory system to hypoxia during disease states at low altitude. 


\section{REFERENCES}

1. Tang Y, Nyengaard JR, Andersen JB, Baandrup U, Gundersen HJ. The application of stereological methods for estimating structural parameters in the human heart. Anat Rec (Hoboken). 2009;292:1630-1647

2. Jayaweera AR, Wei K, Coggins M, Bin JP, Goodman C, Kaul S. Role of capillaries in determining cbf reserve: New insights using myocardial contrast echocardiography. Am J Physiol. 1999;277:H2363-2372

3. Duncker DJ, Merkus D. Acute adaptations of the coronary circulation to exercise. Cell Biochem Biophys. 2005;43:17-35

4. Wexels IC, Myhre ES, Mjos OD. Effects of carbon dioxide and ph on myocardial blood-flow and metabolism in the dog. Clin Physiol. 1985;5:575-588

5. Saitoh S, Zhang C, Tune JD, Potter B, Kiyooka T, Rogers PA, Knudson JD, Dick GM, Swafford A, Chilian WM. Hydrogen peroxide: A feed-forward dilator that couples myocardial metabolism to coronary blood flow. Arterioscler Thromb Vasc Biol. 2006;26:2614-2621

6. Feigl EO. Coronary physiology. Physiol Rev. 1983;63:1-205

7. Berne RM. Cardiac nucleotides in hypoxia: Possible role in regulation of coronary blood flow. Am J Physiol. 1963;204:317-322

8. Brown IP, Thompson Cl, Belloni FL. Role of nitric oxide in hypoxic coronary vasodilatation in isolated perfused guinea pig heart. Am J Physiol. 1993;264:H821-829

9. Penaloza D, Sime F. Circulatory dynamics during high altitude pulmonary edema. Am J Cardiol. 1969;23:369-378

10. Berger MM, Hesse C, Dehnert C, Siedler H, Kleinbongard P, Bardenheuer HJ, Kelm M, Bartsch P, Haefeli WE. Hypoxia impairs systemic endothelial function in individuals prone to high-altitude pulmonary edema. Am J Respir Crit Care Med. 2005;172:763-767

11. Kaufmann BA, Bernheim AM, Kiencke S, Fischler M, Sklenar J, Mairbaurl H, Maggiorini M, Brunner-La Rocca HP. Evidence supportive of impaired myocardial blood flow reserve at high altitude in subjects developing high-altitude pulmonary edema. Am J Physiol Heart Circ Physiol. 2008;294:H1651-1657

12. Murata T, Hori M, Sakamoto K, Karaki H, Ozaki H. Dexamethasone blocks hypoxia-induced endothelial dysfunction in organ-cultured pulmonary arteries. Am J Respir Crit Care Med. 2004;170:647-655

13. Zhao L, Mason NA, Morrell NW, Kojonazarov B, Sadykov A, Maripov A, Mirrakhimov MM, Aldashev A, Wilkins MR. Sildenafil inhibits hypoxia-induced pulmonary hypertension. Circulation. 2001;104:424-428

14. Blume GG, McLeod CJ, Barnes ME, Seward JB, Pellikka PA, Bastiansen PM, Tsang TS. Left atrial function: Physiology, assessment, and clinical implications. Eur J Echocardiogr. 2011;12:421-430

15. Leung DY, Boyd A, Ng AA, Chi C, Thomas L. Echocardiographic evaluation of left atrial size and function: Current understanding, pathophysiologic correlates, and prognostic implications. Am Heart J. 2008;156:1056-1064

16. Douglas PS. The left atrium: A biomarker of chronic diastolic dysfunction and cardiovascular disease risk. J Am Coll Cardiol. 2003;42:1206-1207

17. Tsang TS, Barnes ME, Gersh BJ, Bailey KR, Seward JB. Left atrial volume as a morphophysiologic expression of left ventricular diastolic dysfunction and relation to cardiovascular risk burden. Am J Cardiol. 2002;90:1284-1289

18. Moller JE, Hillis GS, Oh JK, Seward JB, Reeder GS, Wright RS, Park SW, Bailey KR, Pellikka PA. Left atrial volume: A powerful predictor of survival after acute myocardial infarction. Circulation. 2003;107:22072212

19. Rossi A, Cicoira M, Zanolla L, Sandrini R, Golia G, Zardini P, Enriquez-Sarano M. Determinants and prognostic value of left atrial volume in patients with dilated cardiomyopathy. J Am Coll Cardiol. 2002;40:1425

20. Tsang TS, Barnes ME, Gersh BJ, Bailey KR, Seward JB. Risks for atrial fibrillation and congestive heart failure in patients $>/=65$ years of age with abnormal left ventricular diastolic relaxation. Am J Cardiol. 2004;93:54-58 


\section{CHAPTER 7}

21. Osranek M, Fatema K, Qaddoura F, Al-Saileek A, Barnes ME, Bailey KR, Gersh BJ, Tsang TS, Zehr KJ, Seward JB. Left atrial volume predicts the risk of atrial fibrillation after cardiac surgery: A prospective study. J Am Coll Cardiol. 2006;48:779-786

22. Tsang TS, Gersh BJ, Appleton CP, Tajik AJ, Barnes ME, Bailey KR, Oh JK, Leibson C, Montgomery SC, Seward JB. Left ventricular diastolic dysfunction as a predictor of the first diagnosed nonvalvular atrial fibrillation in 840 elderly men and women. J Am Coll Cardiol. 2002;40:1636-1644

23. Tsang TS, Abhayaratna WP, Barnes ME, Miyasaka Y, Gersh BJ, Bailey KR, Cha SS, Seward JB. Prediction of cardiovascular outcomes with left atrial size: Is volume superior to area or diameter? J Am Coll Cardiol. 2006;47:1018-1023

24. Jenkins C, Bricknell K, Marwick TH. Use of real-time three-dimensional echocardiography to measure left atrial volume: Comparison with other echocardiographic techniques. I Am Soc Echocardiogr. 2005;18:991-997

25. Artang R, Migrino RQ, Harmann L, Bowers M, Woods TD. Left atrial volume measurement with automated border detection by 3-dimensional echocardiography: Comparison with magnetic resonance imaging. Cardiovasc Ultrasound. 2009;7:16

26. Buechel RR, Stephan FP, Sommer G, Bremerich J, Zellweger MJ, Kaufmann BA. Head-to-head comparison of two-dimensional and three-dimensional echocardiographic methods for left atrial chamber quantification with magnetic resonance imaging. J Am Soc Echocardiogr. 2013;26:428-435

27. Hunt SA, Abraham WT, Chin MH, Feldman AM, Francis GS, Ganiats TG, Jessup M, Konstam MA, Mancini DM, Michl K, Oates JA, Rahko PS, Silver MA, Stevenson LW, Yancy CW. 2009 focused update incorporated into the acc/aha 2005 guidelines for the diagnosis and management of heart failure in adults a report of the american college of cardiology foundation/american heart association task force on practice guidelines developed in collaboration with the international society for heart and lung transplantation. J Am Coll Cardiol. 2009;53:e1-e90

28. Moss AJ, Zareba W, Hall WJ, Klein H, Wilber DJ, Cannom DS, Daubert JP, Higgins SL, Brown MW, Andrews ML. Prophylactic implantation of a defibrillator in patients with myocardial infarction and reduced ejection fraction. N Engl J Med. 2002;346:877-883

29. Bardy GH, Lee KL, Mark DB, Poole JE, Packer DL, Boineau R, Domanski M, Troutman C, Anderson J, Johnson G, McNulty SE, Clapp-Channing N, Davidson-Ray LD, Fraulo ES, Fishbein DP, Luceri RM, Ip JH. Amiodarone or an implantable cardioverter-defibrillator for congestive heart failure. N Eng/ J Med. 2005;352:225237

30. Vahanian A, Alfieri O, Andreotti F, Antunes MJ, Baron-Esquivias G, Baumgartner H, Borger MA, Carrel TP, De Bonis M, Evangelista A, Falk V, lung B, Lancellotti P, Pierard L, Price S, Schafers HJ, Schuler G, Stepinska J, Swedberg K, Takkenberg J, Von Oppell UO, Windecker S, Zamorano JL, Zembala M. Guidelines on the management of valvular heart disease (version 2012). Eur Heart J. 2012;33:2451-2496

31. Hoffmann R, von Bardeleben S, ten Cate F, Borges AC, Kasprzak J, Firschke C, Lafitte S, Al-Saadi N, KuntzHehner S, Engelhardt M, Becher H, Vanoverschelde JL. Assessment of systolic left ventricular function: A multi-centre comparison of cineventriculography, cardiac magnetic resonance imaging, unenhanced and contrast-enhanced echocardiography. Eur Heart J. 2005;26:607-616

32. Hoffmann R, von Bardeleben S, Barletta G, Pasques A, Kasprzak J, Greis C, Becher H. Comparison of twoand three-dimensional unenhanced and contrast-enhanced echocardiographies versus cineventriculography versus cardiac magnetic resonance for determination of left ventricular function. Am J Cardiol. 2014;113:395-401

33. Effect of enalapril on survival in patients with reduced left ventricular ejection fractions and congestive heart failure. The solvd investigators. N Engl J Med. 1991;325:293-302

34. Effects of metoprolol $\mathrm{cr}$ in patients with ischemic and dilated cardiomyopathy : The randomized evaluation of strategies for left ventricular dysfunction pilot study. Circulation. 2000;101:378-384

35. Kaufmann BA, Min SY, Goetschalckx K, Bernheim AM, Buser PT, Pfisterer ME, Brunner-La Rocca HP. How reliable are left ventricular ejection fraction cut offs assessed by echocardiography for clinical decision making in patients with heart failure? Int J Cardiovasc Imaging. 2013;29:581-588 
36. Liang F, Gardner DG. Mechanical strain activates bnp gene transcription through a p38/nf-kappabdependent mechanism. J Clin Invest. 1999;104:1603-1612

37. von Lueder TG, Sangaralingham SJ, Wang BH, Kompa AR, Atar D, Burnett JC, Jr., Krum H. Reninangiotensin blockade combined with natriuretic peptide system augmentation: Novel therapeutic concepts to combat heart failure. Circ Heart Fail. 2013;6:594-605

38. Maisel AS, Krishnaswamy P, Nowak RM, McCord J, Hollander JE, Duc P, Omland T, Storrow AB, Abraham WT, Wu AH, Clopton P, Steg PG, Westheim A, Knudsen CW, Perez A, Kazanegra R, Herrmann HC, McCullough PA. Rapid measurement of b-type natriuretic peptide in the emergency diagnosis of heart failure. N Engl J Med. 2002;347:161-167

39. Yancy CW, Jessup M, Bozkurt B, Butler J, Casey DE, Jr., Drazner MH, Fonarow GC, Geraci SA, Horwich T, Januzzi JL, Johnson MR, Kasper EK, Levy WC, Masoudi FA, McBride PE, McMurray JJ, Mitchell JE, Peterson PN, Riegel B, Sam F, Stevenson LW, Tang WH, Tsai EJ, Wilkoff BL. 2013 accf/aha guideline for the management of heart failure: A report of the american college of cardiology foundation/american heart association task force on practice guidelines. J Am Coll Cardiol. 2013;62:e147-239

40. Richards AM, Crozier IG, Yandle TG, Espiner EA, Ikram H, Nicholls MG. Brain natriuretic factor: Regional plasma concentrations and correlations with haemodynamic state in cardiac disease. Br Heart J. 1993;69:414-417

41. Troughton RW, Frampton CM, Yandle TG, Espiner EA, Nicholls MG, Richards AM. Treatment of heart failure guided by plasma aminoterminal brain natriuretic peptide ( $n$-bnp) concentrations. Lancet. 2000;355:1126-1130

42. Januzzi JL, Jr., Rehman SU, Mohammed AA, Bhardwaj A, Barajas L, Barajas J, Kim HN, Baggish AL, Weiner RB, Chen-Tournoux A, Marshall JE, Moore SA, Carlson WD, Lewis GD, Shin J, Sullivan D, Parks K, Wang TJ, Gregory SA, Uthamalingam S, Semigran MJ. Use of amino-terminal pro-b-type natriuretic peptide to guide outpatient therapy of patients with chronic left ventricular systolic dysfunction. J Am Coll Cardiol. 2011;58:1881-1889

43. Eurlings LW, van Pol PE, Kok WE, van Wijk S, Lodewijks-van der Bolt C, Balk AH, Lok DJ, Crijns HJ, van Kraaij DJ, de Jonge N, Meeder JG, Prins M, Pinto YM. Management of chronic heart failure guided by individual n-terminal pro-b-type natriuretic peptide targets: Results of the prima (can pro-brain-natriuretic peptide guided therapy of chronic heart failure improve heart failure morbidity and mortality?) study. J Am Coll Cardiol. 2010;56:2090-2100

44. Pfisterer $M$, Buser $P$, Rickli $H$, Gutmann $M$, Erne $P$, Rickenbacher $P$, Vuillomenet A, Jeker U, Dubach $P$, Beer $\mathrm{H}$, Yoon SI, Suter T, Osterhues HH, Schieber MM, Hilti P, Schindler R, Brunner-La Rocca HP. Bnp-guided vs symptom-guided heart failure therapy: The trial of intensified vs standard medical therapy in elderly patients with congestive heart failure (time-chf) randomized trial. JAMA. 2009;301:383-392

45. Di Biase L, Auricchio A, Sorgente A, Civello K, Klersy C, Faletra F, Riedlbauchova L, Patel D, Arruda M, Schweikert RA, Martin DO, Saliba WI, Moccetti T, Wilkoff BL, Natale A. The magnitude of reverse remodelling irrespective of aetiology predicts outcome of heart failure patients treated with cardiac resynchronization therapy. Eur Heart J. 2008;29:2497-2505

46. Hoshikawa E, Matsumura Y, Kubo T, Okawa M, Yamasaki N, Kitaoka H, Furuno T, Takata J, Doi YL. Effect of left ventricular reverse remodeling on long-term prognosis after therapy with angiotensin-converting enzyme inhibitors or angiotensin ii receptor blockers and beta blockers in patients with idiopathic dilated cardiomyopathy. Am J Cardiol. 2011;107:1065-1070

47. Wong M, Staszewsky L, Latini R, Barlera S, Volpi A, Chiang YT, Benza RL, Gottlieb SO, Kleemann TD, Rosconi F, Vandervoort PM, Cohn JN. Valsartan benefits left ventricular structure and function in heart failure: Val-heft echocardiographic study. J Am Coll Cardiol. 2002;40:970-975

48. Bristow MR, Gilbert EM, Abraham WT, Adams KF, Fowler MB, Hershberger RE, Kubo SH, Narahara KA, Ingersoll H, Krueger S, Young S, Shusterman N. Carvedilol produces dose-related improvements in left ventricular function and survival in subjects with chronic heart failure. Mocha investigators. Circulation. 1996;94:2807-2816

49. Streeter DD, Jr., Spotnitz HM, Patel DP, Ross J, Jr., Sonnenblick EH. Fiber orientation in the canine left ventricle during diastole and systole. Circ Res. 1969;24:339-347 


\section{CHAPTER 7}

50. Stuber M, Scheidegger MB, Fischer SE, Nagel E, Steinemann F, Hess OM, Boesiger P. Alterations in the local myocardial motion pattern in patients suffering from pressure overload due to aortic stenosis. Circulation. 1999;100:361-368

51. Takeuchi M, Nishikage T, Nakai H, Kokumai M, Otani S, Lang RM. The assessment of left ventricular twist in anterior wall myocardial infarction using two-dimensional speckle tracking imaging. J Am Soc Echocardiogr. 2007; 20:36-44

52. MacGowan GA, Shapiro EP, Azhari H, Siu CO, Hees PS, Hutchins GM, Weiss JL, Rademakers FE. Noninvasive measurement of shortening in the fiber and cross-fiber directions in the normal human left ventricle and in idiopathic dilated cardiomyopathy. Circulation. 1997;96:535-541

53. Fonseca CG, Dissanayake AM, Doughty RN, Whalley GA, Gamble GD, Cowan BR, Occleshaw CJ, Young AA. Three-dimensional assessment of left ventricular systolic strain in patients with type 2 diabetes mellitus, diastolic dysfunction, and normal ejection fraction. Am J Cardiol. 2004;94:1391-1395

54. Dong L, Zhang F, Shu X, Zhou D, Guan L, Pan C, Chen H. Left ventricular torsional deformation in patients undergoing transcatheter closure of secundum atrial septal defect. Int I Cardiovasc Imaging. 2009;25:479-486

55. Puwanant S, Park M, Popovic ZB, Tang WH, Farha S, George D, Sharp J, Puntawangkoon J, Loyd JE, Erzurum SC, Thomas JD. Ventricular geometry, strain, and rotational mechanics in pulmonary hypertension. Circulation. 2010;121:259-266

56. Kawagishi T. Speckle tracking for assessment of cardiac motion and dyssynchrony. Echocardiography. 2008;25:1167-1171

57. Felker GM, Ahmad T, Anstrom KJ, Adams KF, Cooper LS, Ezekowitz JA, Fiuzat M, Houston-Miller N, Januzzi JL, Leifer ES, Mark DB, Desvigne-Nickens P, Paynter G, Piña IL, Whellan DJ, O'Connor CM.Rationale and Design of the GUIDE-IT Study: Guiding Evidence Based Therapy Using Biomarker Intensified Treatment in Heart Failure. JACC Heart Fail. 2014 Oct;2(5):457-65 
Summary/Samenvatting 


\section{SUMMARY}

The introduction gives an overview of how echocardiography has developed, and explains how the technique can be used to assess cardiac physiology (myocardial perfusion, chamber size and function) and pathophysiology (left ventricular remodeling in response to intensified heart failure treatment).

This thesis then shows how we have used state-of-the-art echocardiography to improve knowledge of left ventricular and left atrial structure, physiology and function. In chapter 2, we show that individuals susceptible to high altitude pulmonary edema have a reduced exercise-induced myocardial blood flow reserve compared with normal individuals, which points to a disturbed endothelial function in the myocardial microcirculation in susceptible individuals. In chapter 6 , we show that changes in left ventricular geometry induced by hypoxia-associated pulmonary artery hypertension at high altitude results in reduced left ventricular torsion. In chapter 4, we validate a novel software tool for dedicated measurement of left atrial size using three-dimensional echocardiography. We show that this tool outperforms. conventional 2D echocardiography in terms of accuracy. Some of the limitations of applying conventional 2Dechocardiography measurements of left ventricular ejection to individual patients are illustrated in chapter 3. In this chapter, we show that the variability in left ventricular ejection fraction measurements could possibly lead to uncertainties for indicating device therapy such as implantable cardioverter defibrillators in up to one fifth of heart failure patients.. Last, echocardiography is used in chapter 5 to shed light on the effects of BNP-guided heart failure therapy on left ventricular remodeling in elderly patients. We show that clinically significant reverse remodeling occurs with intensified medical therapy in heart failure patients independent of age. NT-proBNP-guided treatment significantly interacted with the improvement in left ventricular ejection fraction at all ages suggesting that a larger increase in heart failure therapy is associated with a greater change in left ventricular function, reverse remodelling and as a consequence better outcome than standard therapy, independent of age. 


\section{SAMENVATTING}

De inleiding geeft een overzicht hoe echocardiografie zich de afgelopen decades ontwikkelde en legt uit hoe de techniek kan worden gebruikt om inzicht te krijgen in de fysiologie (myocardiale perfusie, structuur en functie van de hartkamers) en pathofysiologie (remodeling van de linker kamer als antwoord op geïntensiveerde behandeling van hartfalen) van het hart.

De proefschrift laat dan zien hoe wij state-of-the-art echocardiografie toepassen om de kennis te verbeteren van de structuur, fysiologie en functie van de linker kamer en de linker boezem. Hoofdstuk 2 toont dat mensen welke gevoelig ervoor zijn op grote hoogde een longoedeem te ontwikkelen een verminderde bloedstroom van het myocard hebben tijdens inspanning ten opzichte van mensen die niet gevoelig hiervoor zijn. Dit wijst erop dat de endotheliale functie van de myocardiale microcirculatie gestoord is bij deze mensen wat wederom een connectie met pulmonale hypertensie zou kunnen hebben. In hoofdstuk 6 laten wij zien dat veranderingen van de geometrie van de linker kamer veroorzaakt door hypoxie geïnduceerde pulmonale hypertensie tot gereduceerde torsie van de linker kamer leiden. Dit is een interessant model om de interactie tussen de twee kamers bij verhoging van de pulmonaal-druk beter te kunnen begrijpen. In hoofdstuk 4 valideren wij een nieuwe software tool om de structuur van de linker boezem met behulp van drie dimensionale echocardiografie beter te kunnen bepalen. Ten opzichte van conventionele twee dimensionele echocardiografie zijn de resultaten meer betrouwbaar. Vergelijkbare beperkingen heeft ook de twee dimensionele inschatting van de ejectiefractie van de linker kamer. In hoofdstuk 3 laten wij zien hoe groot de variabiliteit van deze metingen zijn, wat tot onzekerheid bij de indicatiestelling van therapieën kan leiden. In ons voorbeeld laten wij zien dat de indicatie of een implanteerbare defibrillator nodig is of niet bij iedere vijfde van onze patiënten met hartfalen anders zou zijn geweest. Tenslotte was echocardiografie gebruikt om meer inzicht te krijgen in de effecten van BNP gestuurde therapie op remodeling van de linker kamer bij hartfalen met verminderde pompfunctie. Wij vonden dat meer geïntensiveerde hartfalen therapie met behulp van NT-proBNP gestuurd tot significant meer reverse remodeling van de linker kamer leidt onafhankelijk van de leeftijd. Onze resultaten suggereren dat een uitbreiden van de hartfalen therapie geassocieerd is met een verbetering van de functie van de linker kamer wat wederom tot een beter uitkomst kan leiden dan standard therapie, onafhankelijk van de leeftijd. 



\section{Acknowledgements}

Being able to do research, to be curious, to learn, to err and try again, to sometimes succeed but fail on probably more occasions, is a tremendous privilege. Hanspeter, thank you for teaching and supporting me, and for giving me the opportunity to enter clinical research - even at 4550 meters above sea level - a fascinating experience for a Swiss with vertigo.

Thanks also to the fellows and staff at the echolab at the University Hospital Basel, you continue to ask the questions I have never asked myself, and I hope you will never stop. Also, I would like to thank Prof. dr. Stefan Osswald for supporting me throughout my career at the University Hospital Basel.

Last but not least my gratitude goes out to my mother and my late dad and of course to my wife Viviana and my children Sophie and Lukas. Thank you for your support and your love. 



\section{Curriculum vitae}

Beat Kaufmann was born on the $17^{\text {th }}$ of august 1971 in Basel, Switzerland. After completing his secondary school at the Gymnasium Liestal in 1990, he started his medical training at the University of Basel, Switzerland and during one year as an Erasmus exchange student at the Universidad Complutense of Madrid, Spain.

After obtaining his medical degree in 1997, he started his medical residency in several hospitals in Switzerland. In 2003 he joined the cardiology department of the University Hospital Basel, where in addition to his clinical education he started work in clinical research under the supervision of prof. dr. Hanspeter Brunner. In 2005 he went to the Oregon Health and Sciences University for a research fellowship in pre-clinical ultrasound. For his work in ultrasound molecular imaging he was awarded the Young Investigator's Award of the American Society of Echocardiography in 2007. Upon returning to the cardiology department of the University Hospital of Basel in 2007, he obtained board certification in Internal Medicine and Cardiology. He was also awarded a SCORE (Swiss Clinicians Opting for Research) fellowship of the Swiss National Science Foundation and continued clinical research with prof. dr. Hanspeter Brunner. Currently, Beat Kaufmann is the head of the adult echocardiography laboratory at the University Hospital of Basel, and the head of the Cardiovascular Molecular Imaging Laboratory at the Department of Biomedicine of the University Hospital of Basel. He continues to conduct research in preclinical ultrasound as well as in clinical ultrasound, and continues to be funded by the Swiss National Science Foundation. 



\section{List of Publications}

\section{ORIGINAL PUBLICATIONS}

1. Pascotto M, Leong-Poi H, Kaufmann BA, Allrogen A, Charalampidis D, Kerut EK, Kaul $\mathrm{S}$, Lindner JR. Assessment of Ischemia-induced Microvascular Remodeling using Contrast-enhanced Ultrasound Vascular Anatomic Mapping. J Am Soc Echocardiogr. 2007;20(9):1100-8.

2. Kaufmann BA, Pfisterer ME, Viswanathan S, Müller-Brand J, Zellweger MJ. Stunning and left ventricular function - how long is the ventricle knocked out? Left ventricular function correlated with ischemic burden and recovery time after stress. Int J Cardiol. 2006 Sep 20;112(2):223-8.

3. Kaufmann BA, Lankford M, Behm CZ, French BA, Klibanov AL, Yaqin Xu, Lindner JR. High-Resolution Myocardial Perfusion Imaging in Mice with High-Frequency Echocardiographic Detection of a Depot Contrast Agent. J Am Soc Echocardiogr. 2007 Feb;20(2):136-43.

4. Kaufmann BA, Lewis C, Xie A, Mirza-Mohd A, Lindner JR. Detection of Recent Myocardial Ischemia by Molecular Imaging of P-selectin with Targeted Contrast Echocardiography. European Heart Journal. 2007;116(3):276-84.

5. Kaufmann BA, Sanders JM, Davis C, Xie A, Sarembock IJ, Lindner JR. Molecular Imaging of Inflammation in Atherosclerosis with Targeted Ultrasound Detecttion of Vascular Cell Adhesion Molecule-1. Circulation. 2007;116(3):276-284.

6. Behm, CZ, Kaufmann BA, Carr C, Lankford M, Sanders JM, Kaul S, Lindner JR. Molecular Imaging of VCAM-1 Expression and Inflammatory Cell Recruitment during Arteriogenesis and Vasculogenesis. Circulation. 2008;117(22):2902-11.

7. Kaufmann BA, Bernheim AM, Kiencke S, Fischler M, Sklenar J, Mairbäurl H, Maggiorini M, Brunner-La Rocca HP. Evidence Supportive of Impaired Myocardial Blood Flow Reserve at High Altitude in Subjects Developing High Altitude Pulmonary Edema. Am J Physiol Heart Circ Physiol. 2008;294(4):H1651-7.

8. Kaufmann BA, Carr CL, Belcik T, Xie A, Kron B, Yu Q, Lindner JR. Effect of Acoustic Power on In Vivo Molecular imaging With Targeted Microbubbles: Implications for 
Low-Mechanical Index Real-Time Imaging. J Am Soc Echocardiogr. 2010 Jan;23(1):79-85.

9. Kaufmann BA, Carr CL, Belcik JT et al. Molecular Imaging of the Initial Inflammatory Response in Atherosclerosis. Implications for Early Detection of Disease. Arterioscler Thromb Vasc Biol. 2010 Jan;30(1):54-9.

10. Rohner A, Brinkert M, Kawel N, Buechel RR, Leibundgut G, Grize L, Kühne M, Bremerich J, Kaufmann BA, Zellweger MJ, Buser P, Osswald S, Handke M. Functional Assessment of the left atrium by real time three-dimensional echocardiography. Eur J Echocardiogr. 2011 Jul;12(7):497-505.

11. Khanicheh E, Mitterhuber M, Kinslechner K, Xu L, Lindner JR, Kaufmann BA. Factors affecting the endothelial retention of targeted microbubbles: Influence of microbubble shell design and cell surface projection of the endothelial target molecule. J Am Soc Echocardiogr. 2012 Apr;25(4):460-6.

12. Belcik JT, Qi Y, Kaufmann BA, Xie A, Bullens S, Morgan TK, Bagby SP, Kolumam G, Kowalski J, Oyer JA, Bunting S, Lindner JR. Cardiovascular and Systemic Microvascular Effects of Anti-Vascular Endothelial Growth Factor Therapy for Cancer. J Am Coll Cardiol. 2012;60(7):618-25

13. Kaufmann BA, Min SY, Goetschalckx K, Bernheim AM, Buser PT, Pfisterer ME, Brunner-La Rocca HP. How reliable are left ventricular ejection fraction cut offs assessed by echocardiography for clinical decision making in patients with heart failure? Int J Cardiovasc Imaging. 2012 Sep 11.

14. Buechel RR, Sommer G, Leibundgut G, Rohner A, Riede F, Kessel-Schaefer A, Kaufmann BA, Zellweger MJ, Bremerich J, Handke M. Assessment of left atrial functional parameters using a novel dedicated analysis tool for real-time three-dimensional echocardiography: validation in comparison to magnetic resonance imaging. Int J Cardiovasc Imaging. 2012 Sep 11.

15. Davidson BP, Kaufmann BA, Belcik JT, Xie A, Qi Y, Lindner JR. Detection of antecedent myocardial ischemia with multiselectin molecular imaging. J Am Coll Cardiol. 2012; Oct 23;60(17):1690-7.

16. Liu YN, Davidsoon BP, Yue Q, Belcik T, Xie A, Inaba Y, McCarty OJ, Tormoen GW, Zhao Y, Ruggeri ZM, Kaufmann BA, Lindner JR. Molecular Imaging of Inflammation and Platelet Adhesion in Advanced Atherosclerosis: Effects of Antioxidant Therapy with NADPH Oxidase Inhibition. Circ Cardiovasc Imaging. 2013 6(1):74-82. 
17. Buechel RR, Stephan FP, Sommer G, Bremerich J, Zellweger MJ, Kaufmann BA. Headto-Head Comparison of Two-Dimensional and Three-Dimensional. Echocardiographic Methods for Left Atrial Chamber Quantification with Magnetic Resonance Imaging. J Am Soc Echocardiogr. 2013 Feb 11. [Epub ahead of print]

18. Sürder D, Pedrazzini G, Gaemperli O, Biaggi P, Felix C, Rufibach K, Maur CA, Jeger R, Buser P, Kaufmann BA, Moccetti M, Hürlimann D, Bühler I, Bettex D, Scherman J, Pasotti E, Faletra FF, Zuber M, Moccetti T, Lüscher TF, Erne P, Grünenfelder J, Corti R. Predictors for efficacy of percutaneous mitral valve repair using the MitraClip system: the results of the MitraSwiss registry. Heart. 2013 Jan 23. [Epub ahead of print]

19. Khanicheh E, Mitterhuber M, Xu L, Haeuselmann SP, Kuster GM, Kaufmann BA. (2013) Noninvasive Ultrasound Molecular Imaging of the Effect of Statins on Endothelial Inflammatory Phenotype in Early Atherosclerosis. PLoS ONE (2013) 8(3): e58761. doi:10.1371/journal.pone.0058761

20. Khanicheh E, Qi Y, Xie A, Mitterhuber M, Xu L, Mochizuki M, Daali Y, Jaquet V, Krause KH, Ruggeri ZM, Kuster GM, Lindner JR, Kaufmann BA. Molecular Imaging Reveals Rapid Reduction of Endothelial Activation in Early Atherosclerosis With Apocynin Independent of Antioxidative Properties.. Arterioscler Thromb Vasc Biol. 2013 Aug 1.

21. Pfister O, Lorenz V, Oikonomopoulos A, Xu L, Häuselmann SP, Mbah C, Kaufmann BA, Liao R, Wodnar-Filipowicz A, Kuster GM.Flt3 activation improves post-myocardial infarction remodeling involving a cytoprotective effect on cardiomyocytes. J Am Coll Cardiol. 2013 Oct 19.

22. Ammon M, Arenja N, Leibundgut G, Buechel RR, Kuster GM, Kaufmann BA, Pfister O. Cardiovascular management of cancer patients with chemotherapy-associated left ventricular systolic dysfunction in real-world clinical practice. J Card Fail. 2013 Sep;19(9):629-34.

23. van Empel VP, Kaufmann BA, Bernheim AM, Goetschalckx K, Min SY, Muzzarelli S, Pfisterer ME, Kiencke S, Maeder MT, Brunner-La Rocca HP. Interaction between pulmonary hypertension and diastolic dysfunction in an elderly heart failure population. J Card Fail. 2014 Feb;20(2):98-104.

24. Kriemler S, Bürgi F, Wick C, Wick B, Keller M, Wiget U, Schindler C, Kaufmann BA, Kohler M, Bloch K, Brunner-La Rocca HP. Prevalence of Acute Mountain Sickness at $3500 \mathrm{~m}$ Within and Between Families: A Prospective Cohort Study. High Alt Med Biol. 2014 Feb 21. 
25. Minners J, Gohlke-Baerwolf C, Kaufmann BA, Bahlmann E, Gerdts E, Boman K, Chambers JB, Nienaber CA, Willenheimer R, Wachtell K, Holme I, Pedersen TR, Neumann FJ, Jander N. Adjusting parameters of aortic valve stenosis severity by body size. Heart. 2014 Apr 29. doi: 10.1136/heartjnl-2013-305225.

26. Studer Bruengger AA, Kaufmann BA, Buser M, Hoffmann M, Bader, Bernheim AM. Diastolic Stress Echocardiography in the Young: a Study in Non-athletic and Endurance-trained Healthy Subjects. J Am Soc Echocardiogr. 2014 Oct;27(10):1053-9.

27. Jander N, Hochholzer W, Kaufmann BA, Bahlmann E, Gerdts E, Boman K, Chambers JB, Nienaber CA, Ray S, Rossebo A, Pedersen TR, Wachtell K, Gohlke-Bärwolf C, Neumann FJ, Minners J. Velocity ratio predicts outcomes in patients with low gradient severe aortic stenosis and preserved EF. Heart. 2014. pii: heartjnl-2014-305763. doi: 10.1136/heartjnl-2014-305763. [Epub ahead of print]

28. Soliman D, Bolliger D, Skarvan K, Kaufmann BA, Lurati Buse G, Seeberger MD. Intraoperative assessment of pulmonary artery pressure by transoesophageal echocardiography. Anaesthesia. 2014 Nov 10. doi: 10.1111/anae.12920. [Epub ahead of print]

29. Zimmermann AJ, Bossard M, Aeschbacher S, Schoen T, Voellmin G, Suter $Y$, Lehmann A, Hochgruber T, Pumpol K, Sticherling C, Kühne M, Conen D, Kaufmann BA. Effects of sinus rhythm maintenance on left heart function after electrical cardioversion of atrial fibrillation: implications for tachycardia-induced cardiomyopathy. Can J Cardiol. 2015 Jan;31(1):36-43.

30. Buechel RR, Kaufmann BA, Tobler D, Wild D, Zellweger MJ. Non-invasive nuclear myocardial perfusion imaging improves the diagnostic yield of invasive coronary angiography. Eur Heart J Cardiovasc Imaging. 2015 Apr 26.

31. Kaufmann BA, Goetschalckx K, Min SY, Maeder MT, Bucher U, Nietlispach F, Bernheim AM, Pfisterer ME, Rocca HP; TIME-CHF investigators. Improvement in left ventricular ejection fraction and reverse remodeling in elderly heart failure patients on intense NT-proBNP-guided therapy. Int J Cardiol. 2015 Jul 15;191:286-93

32. Reuthebuch O, Koechlin L, Kaufmann BA, Kessel-Schaefer A, Gahl B, Eckstein FS. Transapical Transcatheter Aortic Valve Implantation Using the JenaValve: A OneYear Follow-up. Thorac Cardiovasc Surg. 2015 Jun 1 


\section{REVIEWS}

1. Kaufmann BA, Kaiser C, Pfisterer ME, Bonetti PO. Coronary stent infections: a rare but severe complication of percutaneous coronary intervention. Swiss Med Wkly. 2005 Aug 20;135(33-34):483-7. Review.

2. Kaufmann BA, Wei K, Lindner JR. Contrast echocardiography. Curr Probl Cardiol. 2007 Feb;32(2):51-96. Review.

3. Kaufmann BA, Lindner JR. Molecular imaging with targeted contrast ultrasound. Curr Opin Biotechnol. 2007 Feb;18(1):11-6. Review.

4. Kaufmann BA. Ultrasound Molecular Imaging of Atherosclerosis. Cardiovascular Research 2009. Sep 1;83(4):617-25. Review.

5. Kaufmann BA Ultrasound Molecular Imaging of Cardiovascular Disease Curr Cardiovasc Imaging Rep (2010) 3:18-25. Review.

6. Nägele R, Kaufmann BA. Praxis (Bern 1994). 2013 Oct 1;102(22):1361-70.

7. Steinl DC, Kaufmann BA. Ultrasound Imaging for Risk Assessment in Atherosclerosis. Int J Mol Sci. 2015 Apr 29;16(5):9749-9769. 


\section{CASE REPORTS}

1. Kaufmann BA, Cahenzli C, Zehnder B, Schoenenberger R. Hyponatremia of unknown origin in an otherwise healthy young woman, case report. Swiss Med Forum 2003;3:794-796

2. Blaich A, de Roche M, Kaufmann BA, Suter-Riniker F, Rosin C, Frei R, Weisser M. Endocarditis due to a stealthy bug. Int J Cardiol. 2012 Oct 18;160(3):e54-5.

3. Nietlispach F, Eckstein F, Seeberger M, Osswald S, Kaufmann BA, Reuthebuch O Closure of Apical Access Site After Transapical, Transcatheter Paravalvular Leak Closure. Can J Cardiol. 2012 Jul-Aug;28(4):516.e5-7.

4. Moccetti F, Kaufmann BA, Tobler D. Differential clubbing and cyanosis: a pathognomonic finding in cardiology. Eur Heart J. 2014 Feb 7.

5. Kurz M, Kaufmann BA, Baddour L, Widmer AF. Propionibacterium acnes prosthetic valve endocarditis with abscess formation: a case report. BMC Infect Dis. 2014 Feb 25;14(1):105. 